\title{
Reduced-Order Aerothermoelastic Framework for Hypersonic Vehicle Control Simulation
}

\author{
Nathan J. Falkiewicz* and Carlos E. S. Cesnik ${ }^{\dagger}$ \\ Department of Aerospace Engineering, University of Michigan, Ann Arbor, Michigan 48109-2140, USA \\ Andrew R. Crowell ${ }^{\ddagger}$ and Jack J. McNamara ${ }^{\S}$ \\ Department of Aerospace Engineering, The Ohio State University, Columbus, Ohio 43210-1226, USA
}

\begin{abstract}
Hypersonic vehicle control system design and simulation requires models that contain a low number of states. Modeling of hypersonic vehicles is complicated due to complex interactions between aerodynamic heating, heat transfer, structural dynamics, and aerodynamics in the hypersonic regime. Though there exist techniques for analyzing the effects of each of the various disciplines, these methods often require solution of large systems of equations which is infeasible within a control design and evaluation environment. This work therefore presents an aerothermoelastic framework with reduced-order aerothermal, heat transfer, and structural dynamic models for time-domain simulation of hypersonic vehicles. The problem is outlined and aerothermoelastic coupling mechanisms are described. Details of the reduced-order models are given and a representative hypersonic vehicle control surface to be used for the study is described. The error between the reduced-order models is characterized by comparison with high-fidelity models. The effect of aerothermoelasticity on total lift and drag is studied and is found to result in up to $8 \%$ change in lift and $21 \%$ change in drag with respect to a rigid control surface for the four trajectories considered. An iterative routine is used to determine the necessary angle of attack needed to match the lift of the deformed control surface to that of a rigid one at successive time instants. Application of the routine to different cruise trajectories shows a maximum departure from the initial angle of attack of $7 \%$.
\end{abstract}

\section{Nomenclature}

*Ph.D. Candidate, Dept. of Aerospace Engineering, 1320 Beal Ave, Ann Arbor, MI 48109, Student Member AIAA. $\dagger$ Professor of Aerospace Engineering, Dept. of Aerospace Engineering, University of Michigan, 1320 Beal Ave, Ann Arbor, MI 48109, Associate Fellow AIAA.

${ }^{\ddagger}$ Ph.D. Candidate, Dept. of Aerospace Engineering, 2036 Neil Ave, Columbus, Ohio 43210, Student Member AIAA.

$\S$ Assistant Professor of Aerospace Engineering, Dept. of Aerospace Engineering, The Ohio State University, 2036 Neil Ave, Columbus, Ohio 43210, Senior Member AIAA. 


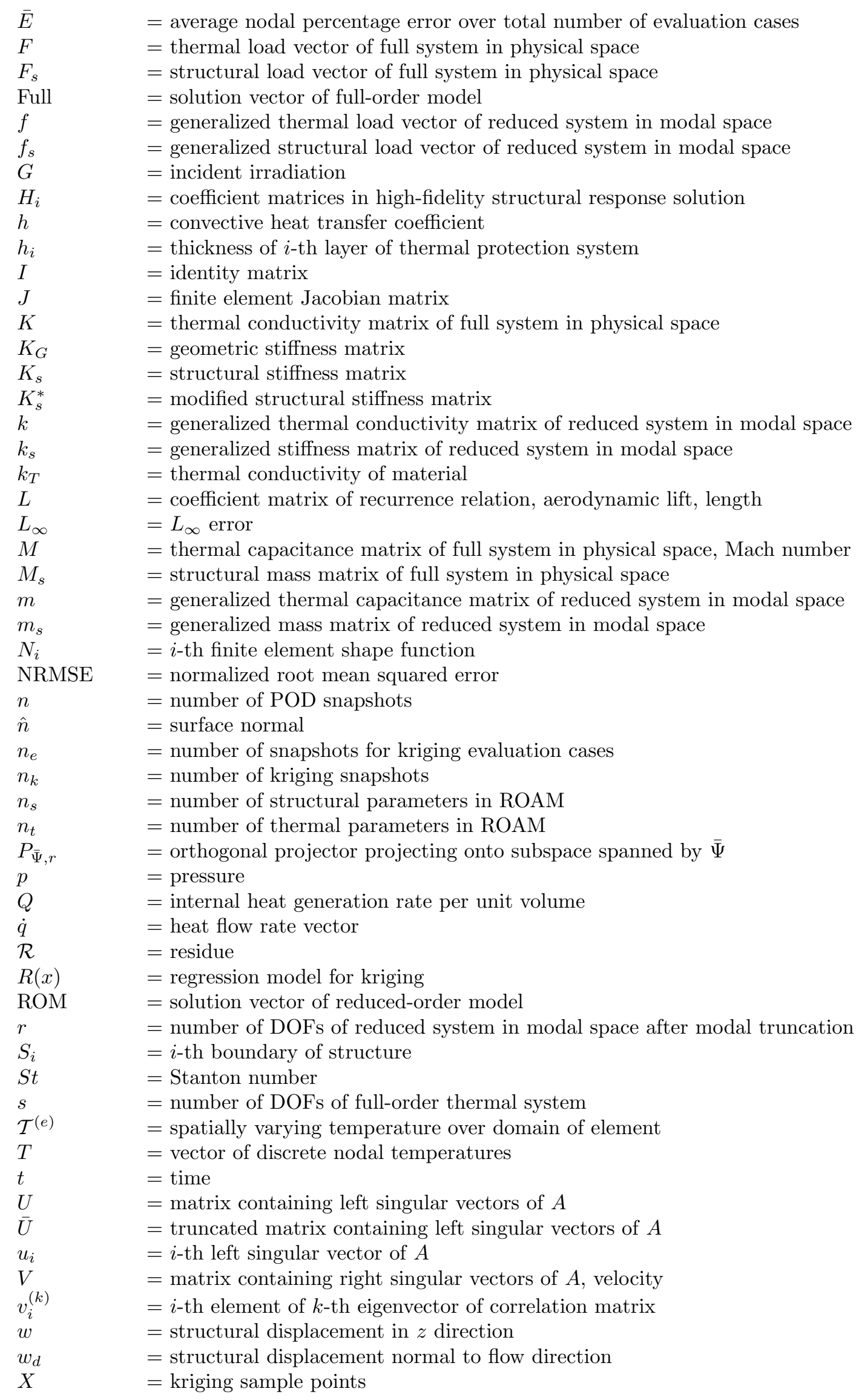




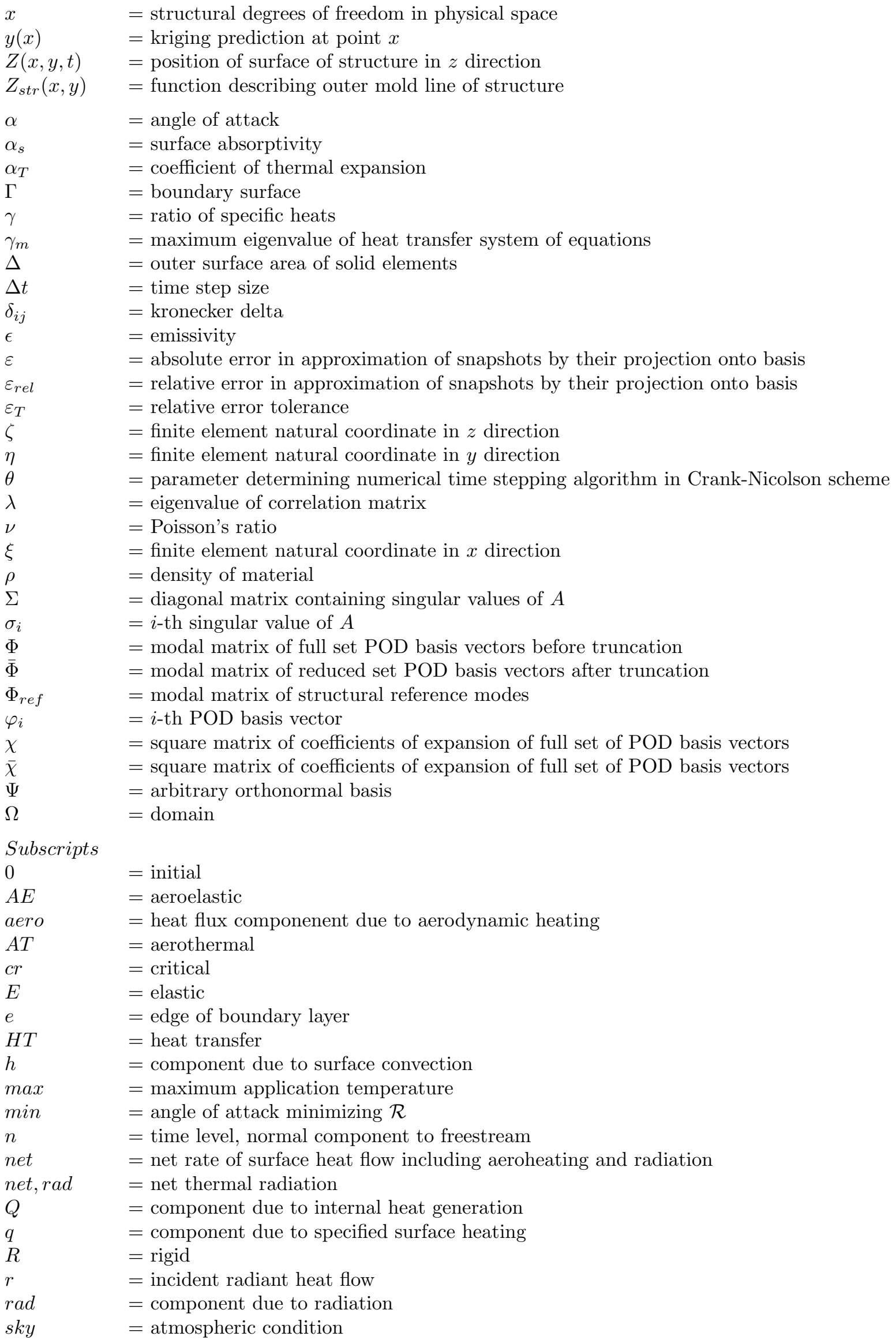




$$
\begin{aligned}
& \text { solar } \quad=\text { solar condition } \\
& T=\text { component due to specified nodal temperature } \\
& \text { Tri }=\text { two-dimensional triangular element } \\
& t \quad=\text { total or stagnation quantity } \\
& \text { Wedge } \quad=\text { three-dimensional wedge element } \\
& w \quad=\text { wall } \\
& \sigma \quad=\text { component due to thermal radiation } \\
& \infty \quad=\text { freestream }
\end{aligned}
$$

Superscripts

(e) $\quad=$ finite element domain

$T \quad=$ matrix transpose

\section{Introduction}

Design and simulation of hypersonic vehicle (HSV) control systems requires consideration of a variety of disciplines due to the highly coupled nature of the flight regime. ${ }^{1-4}$ In order to capture all of the potential effects on vehicle controllability, one must consider the aerodynamics, aerodynamic heating, heat transfer, and elastic airframe as well as the interactions between these disciplines. The problem is further complicated by the high computational expense involved in capturing all of these effects and their interactions in a fullorder sense. Aerothermoelastic modeling is particularly challenging for control system design and evaluation due to the need for models with a low number of states for this type of analysis. While high-fidelity modeling techniques exist for each of these disciplines, the use of such techniques is computationally infeasible in a control design and simulation setting for such a highly coupled problem. Early in the design stage, many iterations of analyses may need to be carried out as the vehicle design matures, thus requiring quick analysis turn-around time. Additionally, the number of states and number of degrees of freedom used in the analyses must be small enough to allow for efficient control simulation and design. As a result, alternative approaches must be considered for vehicle simulations. There are two methodologies that can be utilized in the generation of low order models. The first approach is to apply simplifying assumptions that enable the use of first-principles models. These models are characterized by their low-order form and they can often be solved analytically, thus preventing the need to time-march the solution. While these models are useful in generating a low order representation of the physics, the simplifying assumptions made to employ these models often preclude the ability to model detailed geometries or complex physics. This work will therefore make use of an alternative approach which involves the use of reduced-order models (ROMs) that are derived from high-fidelity analysis tools. Use of high-fidelity tools alone is infeasible due to their high order and long run time. Thus, this work seeks to go beyond simply coupling existing high-fidelity codes and routines, and instead proposes to use the output of these tools along with model reduction techniques to generate computationally tractable systems of governing equations. While aerothermoelastic analysis for control system design has been performed previously, many simplifying assumptions have been used to reduce the size of the problem. The objective of the current work is to make aerthermoelastic simulation of complex geometries feasible for control design without the need for a priori assumptions regarding the physics of the problem. As opposed to a first-principles modeling approach, by first modeling as much of the physics as possible and then systematically reducing the order of the system, one can control and quantify the error incurred through model reduction. This also allows for tailoring of the number of states and degrees of freedom as different levels of fidelity may be required for control system design and evaluation.

This work focuses on the coupling of the aerodynamics, aerodynamic heating, heat transfer, elastic airframe and vehicle dynamics and control. The overall goal of this work is to provide a reduced-order modeling framework that can be used to assess the robustness of the HSV control effector to aerothermoelastic effects in an efficient manner. To do so, careful consideration of the coupling mechanisms between the various disciplines is essential. A flowchart illustrating the coupling of these disciplines is shown in Fig. 1. Due to the high speed involved in hypersonic flight, stagnation effects and the turbulent boundary layer lead to the existence of an aerodynamic heat flux at the surface of the vehicle. To calculate the spatially and temporally varying heat flux, it is necessary to know the aerodynamic flow properties over the vehicle. Note the two-way coupling between the aerodynamic heat flux and the transient temperature distribution. The upward pointing arrow illustrates the fact that the aerodynamic heat flux represents a thermal boundary condition for the heat transfer problem. The downward pointing arrow indicates the fact that the heat flux 
is dependent on the wall temperature of the structure. Additionally, thermal radiation between the outer surface of the structure and the environment must be included. A two-way coupling also exists between the radiation and temperature distribution. Once the aerodynamic problem is solved and the heat flux and radiation flux are known, the boundary conditions for the heat transfer problem are generated. The transient thermal problem is then solved, resulting in a spatially varying temperature distribution. The loads on the structure will have two components: thermal loads resulting from differential thermal expansion of the structure and aerodynamic pressure loads. Additionally, heating of the structure results in changing stiffness distribution due to temperature-dependence of material properties such as Young's Modulus. The development of thermal stresses results in a further change in the stiffness. As a result of the loads, the structure will displace relative to its undeformed configuration. Deformation of the structure leads to a modified aerodynamic profile and the aerodynamic flow properties over the vehicle will change. The flow properties must then be re-calculated as they will affect the loads on the structure, the aerodynamic heat flux, and the aerodynamic forces and moments on the vehicle. Once the updated aerodynamic flow parameters are known, the pressures can be integrated over the vehicle to calculate the resultant forces and moments on the vehicle. Based on the forces and moments, the vehicle equations of motion are propagated and necessary control inputs (i.e., control surface deflections) are determined based on the commanded trajectory and vehicle performance. The control surface deflections in turn change the aerodynamic flow parameters. Once the control inputs are determined, the process is repeated at the next time step. Because the control surfaces are expected to provide a significant contribution to the aerodynamic lift, drag, and moments acting on the vehicle, thorough analysis of the major couplings involved with such a structure is required in order to accurately predict vehicle performance.

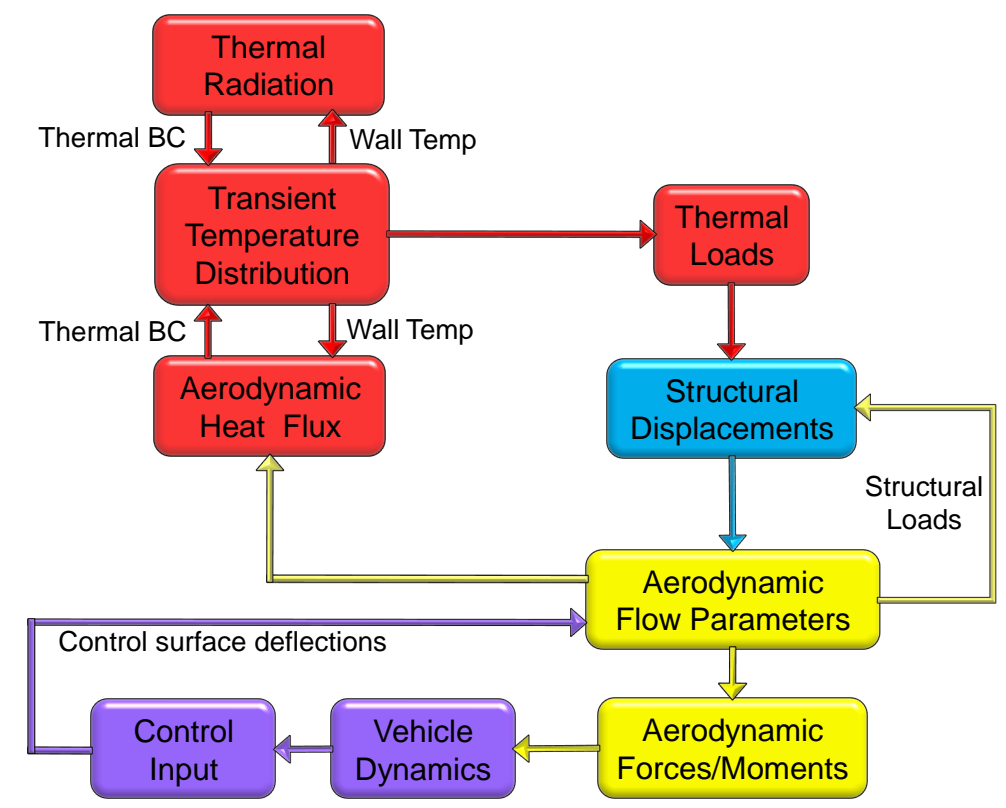

Figure 1. Coupling between aerodynamic heating, heat transfer, elastic airframe, aerodynamics, and vehicle dynamics.

\section{I.A. Previous Work on Aerothermoelastic Modeling}

Previous research in the area of hypersonic aerothermoelasticity has largely utilized high-fidelity analysis tools or analytical models for the various disciplines involved. Analytical models of various aspects of the aerothermoelastic problem have been widely used for control-oriented modeling of hypersonic vehicles. ${ }^{2,5-12}$ The closed-form nature of these models allows for characterization of the vehicle dynamics early in the design cycle and permits evaluation of stability derivatives more readily than with numerical models. The first effort to develop a comprehensive analytical model was conducted by Chavez and Schmidt. ${ }^{5}$ This work used Newtonian impact theory for the aerodynamic pressures, one-dimensional aero/thermo analysis for the propulsion system, and a lumped-mass modal model for the structural dynamics. A subsequent work ${ }^{6}$ employed a Lagrangian approach to capture the elastic deformation, fluid flow, rotating machinery, and spherical Earth. The resulting equations of motion were derived and a preliminary study of the significance 
of selected terms in the equations was presented. Another work ${ }^{2}$ presents a nonlinear physics-based model of the longitudinal dynamics for an air-breathing HSV. Oblique shock and Prandtl-Meyer expansion theory were used for the aerodynamics and the structure was modeled as two beams clamped at the center of mass of the fuselage. While these closed-form approaches provide tractable systems of equations for control-oriented analysis, their ability to capture coupled aerothermoelastic effects for complex geometries with a high level of accuracy is limited.

A variety of studies have focused on the aerothermoelastic response and stability of panels with various degrees of aerothermoelastic coupling. A review paper summarized various analytical methods for nonlinear panel flutter at supersonic and hypersonic speeds. ${ }^{13}$ The methods are grouped into two main areas: 1) classical methods including Galerkin in conjunction with numerical integration, harmonic balance, and perturbation methods, and 2) finite element methods in the frequency or time domain. Two specific studies ${ }^{14,15}$ considered nonlinear panel flutter for high-Mach-number flows under aerothermoelastic effects. These studies both made use of von Kármán plate theory for large displacements and both include geometric nonlinearity due to compressive load. The panel temperature was taken to be equal to the instantaneous flow temperature and was assumed to be uniform to allow for a lumped-capacity approach to the solution of the heat transfer equations. Two related works used an explicit Taylor-Galerkin algorithm to solve the coupled fluid-thermalstructural equations to assess the impact of aerothermoelastic effects on leading edges ${ }^{16}$ and panels. ${ }^{17}$ These works employed an integrated finite element approach which solved the Navier-Stokes equations, energy equation, and quasi-static structural equations of motion in an integrated framework. Results from both works showed that structural deformation has a strong effect on flow properties and that severe aerodynamic heating can lead to large stresses due to steep temperature gradients. Another work ${ }^{18}$ focused on the thermal analysis of thermal protection panels in hypersonic flow under an imposed deflection. The framework consisted of a finite volume Navier-Stokes CFD code coupled with a boundary element formulation of the transient heat transfer process. The ability of the framework to handle deflection was assessed by imposing a deformed structural configuration, calculating the thermal boundary conditions over the deformed panel, and solving for the transient temperature distribution.

Another research area in which aerothermoelasticity in high-speed flows has been studied widely is the area of functionally graded panels. ${ }^{19-24}$ These works include aerothermoelastic effects to varying extents and are motivated largely by the need to assess the response of the structure as well as its aerothermoelastic stability. Praveen and Reddy ${ }^{19}$ include thermal effects by imposing temperatures at the ceramic-rich and metal-rich surfaces and solving a simple steady state heat transfer problem to obtain the through-thickness temperature distribution of the plate. Temperature was taken to be uniform in the plane of the plate. Structural equations of motion were based on a combination of first-order plate theory and von Kármán strains. Static and dynamic finite element analysis was performed and the plate deflections and stresses were analyzed. Prakash and Ganapathi ${ }^{24}$ also included thermal loads by imposing temperatures at the outer surfaces of the structure. This work investigated the influence of thermal loads on the supersonic flutter behavior of functionally graded flat panels. In contrast to Ref. 19, this study included aerodynamic loads by considering the first-order high-Mach number approximation to linear potential flow theory. The equations of motion were formulated using Lagrange's equations and eigenvalue solutions were used to assess the critical aerodynamic pressure for flutter. A later work ${ }^{23}$ extended the study of Ref. 24 by using piston theory to incorporate quasi-steady aerodynamic loads, however, this study did not include thermal effects. In a related work, ${ }^{22}$ thermal effects were incorporated along with the quasi-steady piston theory formulation. The temperature distribution was again obtained by considering temperature variation in the thickness direction only, specifying temperatures at the upper and lower surfaces, and solving the steady state heat transfer equation subject to these Dirichlet boundary conditions. Two other works ${ }^{20,21}$ also utilized quasi-steady first-order piston theory and included thermal effects by imposing a uniform temperature change on the plate. While these works provide progress on aerothermoelastic modeling in high-speed flows, the solution of the heat transfer problem by assuming surface temperatures, using a state state thermal solutions, or semi-empirical methods is insufficient for the problem at hand. Additionally, the current work will use an unsteady aerodynamic solution as opposed to a quasi-steady one.

Recent research on aerothermoelastic stability of a hypersonic vehicle control surface used computational fluid dynamics (CFD) to compute the aerodynamic heating along with finite element thermal and structural models to assess its behavior in hypersonic flow. ${ }^{25}$ In these studies the aerodynamic heat flux was found by using rigid-body CFD solutions to compute the adiabatic wall temperature and heat transfer coefficient at the surface of the structure. The resulting thermal boundary conditions were applied to a transient 
thermal finite element analysis and the resulting temperatures were applied to the structural dynamic finite element model. Free vibration frequencies and mode shapes of the heated structure were calculated under both material property degradation with temperature and thermal stresses at each desired point in time. The mode shapes were used in a modal representation of the equations of motion to obtain the aeroelastic response. Third-order piston theory and Euler aerodynamics were used to obtain the generalized loads. The use of CFD for solution of the aeroheating problem provides capability for capturing complex effects such as shock/boundary layer interaction and 3-D flow effects. However, the computational cost associated with CFD is prohibitive in the control design and simulation setting, and the use of an aerothermal ROM is warranted. While Ref. 25 reduced the order of the equations of motion by applying a truncated set of free vibration mode shapes, an eigenvalue solution was still computed at each desired point in time to update the mode shapes. The current work extends that methodology by using a fixed basis throughout the simulation to avoid the need to solve an eigenvalue problem during the course of the simulation while still reducing the order of the equations of motion.

In two recent studies by Culler et.al., ${ }^{26,27}$ the flow-thermal-structural coupling of two and three dimensional skin panels in hypersonic flow was performed. In Ref. 26 two dimensional panel flutter analysis of a 2-D simply supported HSV skin panel was performed using von Kármán plate theory for the structural dynamic response, piston theory for the unsteady aerodynamics, and Eckert's reference enthalpy method for the aerodynamic heating. The most important coupling mechanism on the flutter boundary was found to be the effect of material property degradation due to elevated temperature. The effect of two-way coupling was found to increase the rate of material degradation in localized regions, thus reducing the flight time to onset of flutter. Additionally, it was found that inertial effects have little impact on deformation prior to flutter and that including inertial effects in the pre-flutter analysis has a small effect on flutter boundary predictions. In Ref. 27 a similar study was carried out to assess the effect of aerothermoelasticity on a 3-D HSV skin panel. A 3-D finite element model was used to obtain the structural dynamic response as opposed to von Kármán plate theory. Loads on the structure consisted of aerodynamic pressure loads, fluctuating pressure loads due to sound pressure level in the turbulent boundary layer, and thermal loads due to temperature gradients. Results demonstrated that the signficance of fluid-thermal-structural coupling for quasi-static response prediction is a strong function of in-plane boundary conditions. Potential for panel failure was found to result from uncertainty in structural boundary conditions.

The current work builds on the previously described literature in a number of areas. The framework includes all aspects of the aerothermoelastic problem in a fully coupled, unsteady sense. This includes transient thermal boundary conditions that are updated based on instantaneous flow conditions and wall temperature. As numerical models are used instead of analytical models, the consideration of complex geometry and coupled physics is made possible. Furthermore, reduced-order modeling techniques are employed to reduce the order of the original models and make the framework computationally tractable. The thermoelastic portion of the work is a continuation of previous studies on reduced-order modeling of the heat transfer and structural dynamics problems. ${ }^{28-30}$ The earliest of these ${ }^{30}$ introduced the reduced-order thermoelastic modeling framework which utilized Proper Orthogonal Decomposition (POD) for reduction of the thermal problem and a modified modal method for reduction of the structural dynamics problem. The framework was applied to a representative HSV structure and results demonstrated the accuracy of the POD formulation as well as the coupling of the equations of motion that results due to heating. A subsequent work $^{29}$ employed a quasi-steady aerothermoelastic time-marching procedure to assess the effect of thermal loads on the aerodynamic forces over a control surface. A more recent work ${ }^{28}$ described an extension of the previous works to unsteady form and specifically addressed the use of POD with time-dependent boundary conditions. The current work makes use of the thermoelastic portion of the framework described in Ref. 28 along with a reduced-order aerothermal model based on CFD solutions.

The aerothermal model used for calculation of the aerodynamic heat flux at the surface of the structure is based on that developed in previous studies. ${ }^{31-34}$ The first three of these works ${ }^{31-33}$ applied a POD-based approach to study the aerothermoelastic response of a von Kármán panel. A more recent work ${ }^{34}$ applied both a POD basis and a surrogate-based function constructed from kriging using Latin Hypercube parameter space sampling. The models were generated by performing full-order solutions at various points within the identified parameter space. Results demonstrated the ability of the approach to enable an accurate, robust, and efficient method for incorporating CFD aerodynamic heating predictions into aerothermoelastic analysis. A similar kriging model to that of Ref. 34 is extended in the current work by incorporating the aerodynamic heating model into the aerothermoelastic ROM framework. 


\section{I.B. Aerothermoelastic Simulation Framework}

The progress described above has led to further investigation into the aerothermoelastic coupling exhibited in hypersonic flight. A flowchart of overall framework applied in this work is shown in Fig. 2. The process begins with the calculation of the heat flux on the outer surface of the structure at the initial time. With the boundary conditions and initial conditions of the thermal problem known, the transient temperature distribution is marched forward in time. Solution of the heat transfer problem is carried out in modal space using modes from POD (to be described in a later section) to avoid the computational cost of running fullorder finite element analysis. The bypassing of the full-order thermal solution via the reduced-order solution is indicated by the gray blocks. This work considers three coupling mechanisms between the thermal and structural solutions. The first involves the thermal stresses that occur in the structure due to differential thermal expansion resulting from the spatially varying temperature distribution. The second is due to the temperature-dependence of the Young's modulus resulting from the high temperatures experienced in hypersonic flight. The third involves the thermal loads that are generated on the structure due to thermal expansion. With the stiffness and structural loads known, the structural dynamics system of equations in physical space is transformed to a suitable reduced modal basis to be described in a subsequent section. The reduced modal system is then solved for the modal coordinates to obtain the structural response. The structural deformations couple with the aerothermal problem due to the effect on aerodynamic flow properties, which change the heat flux. The deformations also result in a change in aerodynamic pressures which modify the structural loads. With the deformed configuration known at the current time step, the aerodynamic flow parameters and heat flux are recalculated and the procedure is repeated at the next time instant.

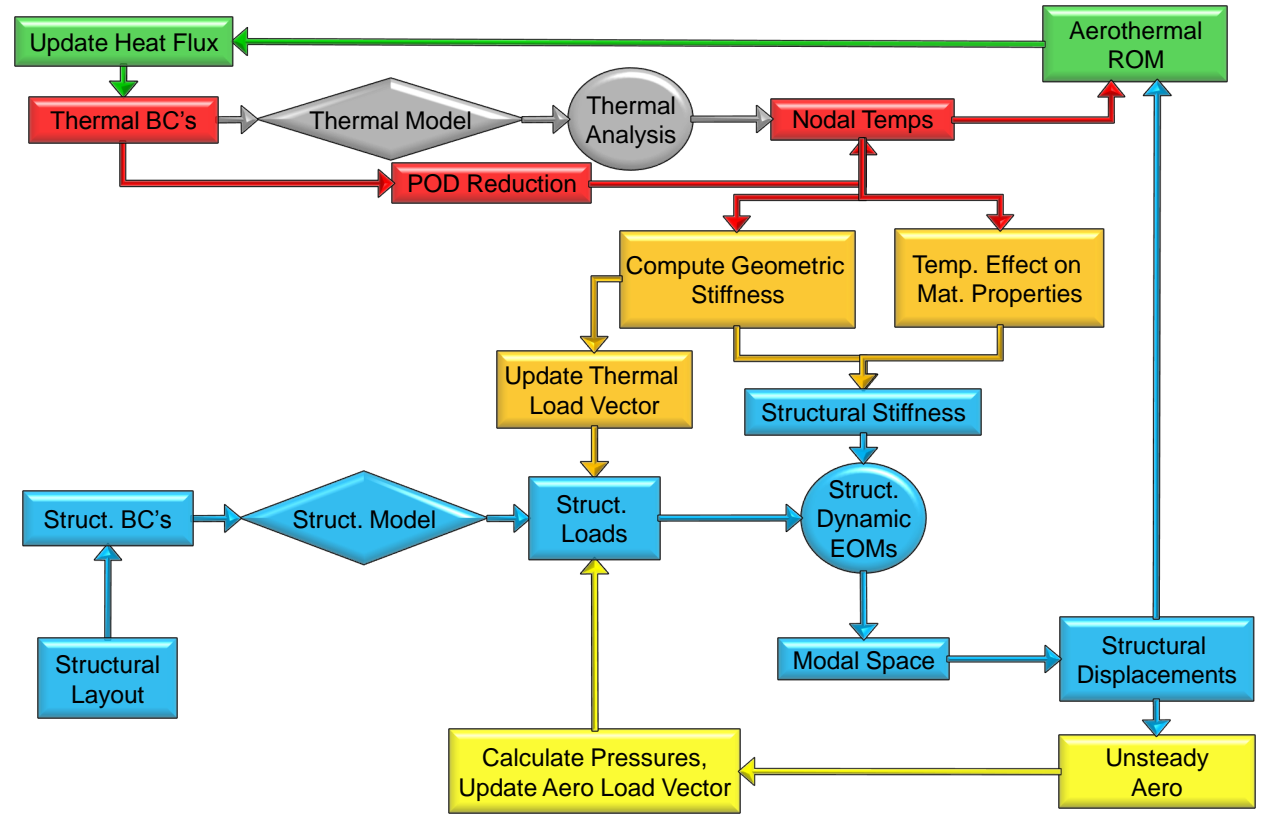

Figure 2. Reduced-order aerothermoelastic modeling framework implemented in this work. 


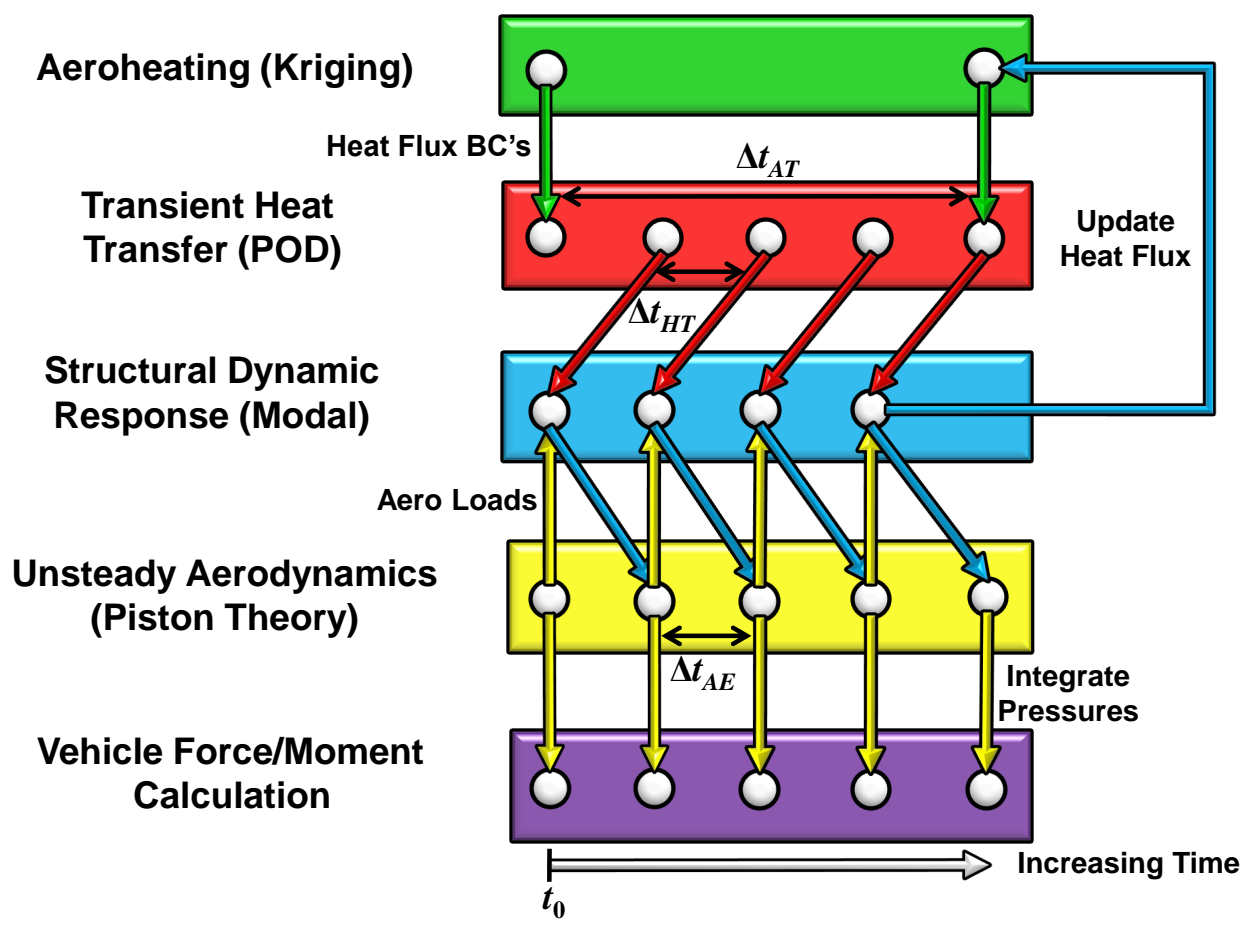

Figure 3. Overview of aerothermoelastic time-stepping schedule.

A time-marching procedure with updates to the thermal and structural boundary conditions at specified intervals is proposed for solution of the coupled aerothermoelastic problem. An outline of the time-stepping schedule is given in Fig. 3. A fundamental assumption to be investigated in the study is that the size of the aeroelastic time step, $\Delta t_{A E}$, will be smaller than the size of the aerothermal time step, $\Delta t_{A T}$. The procedure begins by calculating the aerodynamic flow properties over the undeformed structure at initial time, $t_{0}$. Using the flow properties, the heat flux at the outer surface is found along with the local skin friction coefficients. The aerodynamic pressures and viscous drag components are then integrated to determine the aerodynamic forces and moments at initial time. With the thermal boundary conditions known, a pre-determined number of thermal time steps are taken, each of size $\Delta t_{H T}$, until the time $t_{0}+\Delta t_{A T}$ is reached. The thermal loads based on the temperature change between $t_{0}$ and $t_{0}+\Delta t_{A E}$ are then applied to the structural configuration at $t_{0}$. Additionally, the aerodynamic loads based on the already calculated flow properties are applied to the structure. The structural deformations are then calculated. The displacements are fed back into the aerodynamic solver and the flow properties are calculated at time $t_{0}+\Delta t_{A E}$ over the updated deformed configuration. Each time the flow properties are re-calculated, the aerodynamic pressures are also integrated to allow for characterization of the transient aerodynamic forces and moments on the vehicle. The aeroelastic iterations continue to be carried for a pre-determined number of time steps. Once the time instant $t_{0}+\Delta t_{A T}$ has been reached, the time history of the structural dynamic response is used to update the aerodynamic heat flux. The coupling between the aerothermal and aeroelastic solutions is performed in a time-averaged dynamic $^{26}$ sense in which the time-average of the structural dynamic response in between aerothermal updates is used in the calculation of the thermal boundary conditions. The details of how this information is used to update the heat flux are given in a later section. With the updated thermal boundary conditions known, the transient thermal solution is marched forward from the time instant $t_{0}+\Delta t_{A T}$ to the time instant $t_{0}+2 \Delta t_{A T}$ and the process is repeated. The following sections describe the formulations for solution of the various aspects of the aerothermoelastic problem. 


\section{Transient Thermal Modeling}

\section{II.A. High-Fidelity Transient Thermal Solution}

The finite element formulation for the heat transfer problem begins with the governing energy equation for heat transfer in a three-dimensional anisotropic solid $\Omega$ bounded by outer surface $\Gamma$ given by ${ }^{35}$

$$
-\left(\frac{\partial \dot{q}_{x}}{\partial x}+\frac{\partial \dot{q}_{y}}{\partial y}+\frac{\partial \dot{q}_{z}}{\partial z}\right)+Q=\rho c_{p} \frac{\partial T}{\partial t},
$$

where $\dot{q}_{x}, \dot{q}_{y}$, and $\dot{q}_{z}$ are the components of the heat flow rate vector in cartesian coordinates, $Q$ is the volumetric internal heat generation rate, $\rho$ is the density of the structure, $c_{p}$ is the specific heat of the structure, $T$ denotes temperature, and $t$ denotes time. For an isotropic medium, the components of the heat flow rate vector are given by

$$
\dot{q}=-k_{T} \nabla T,
$$

where $k_{T}$ is the thermal conductivity of the material. Note that the minus sign is needed due to the fact that heat is transferred from warmer regions to cooler regions. Substituting Eq. (2) into Eq. (1) and assuming constant thermal properties and no internal heat generation yields the heat equation given by

$$
\nabla^{2} T=\frac{1}{\alpha_{T}} \frac{\partial T}{\partial t},
$$

where $\alpha_{T}$ is the thermal diffusivity given by

$$
\alpha_{T}=\frac{k_{T}}{\rho c_{p}} .
$$

Now that the governing equations have been given, we will derive the resulting finite element equations for a single element for simplicity. Using the method of weighted residuals with the governing equation, we require

$$
\int_{\Omega^{(e)}}\left(\frac{\partial \dot{q}_{x}}{\partial x}+\frac{\partial \dot{q}_{y}}{\partial y}+\frac{\partial \dot{q}_{z}}{\partial z}-Q+\rho c_{p} \frac{\partial T}{\partial t}\right) N_{i} d \Omega=0,
$$

where $\Omega^{(e)}$ is the domain for element $(e)$ and $N_{i}$ are the element shape functions. As the finite element model used in this work contains two-dimensional triangular elements and three-dimensional wedge elements, the formulation will require the shape functions for both element types. The shape functions for the twodimensional triangular element are given by ${ }^{36}$

$$
\begin{aligned}
& N_{1, T r i}=\xi \\
& N_{2, T r i}=\eta \\
& N_{3, T r i}=1-\xi-\eta,
\end{aligned}
$$

where $\xi$ and $\eta$ are the natural coordinates of the local element coordinate system in the $x$ and $y$ directions, respectively, each with limits from 0 to 1 . The shape functions for the three-dimensional wedge element are given by 37

$$
\begin{aligned}
& N_{1, \text { Wedge }}=(1-\xi-\eta)(1-\zeta) / 2 \\
& N_{2, \text { Wedge }}=\xi(1-\zeta) / 2 \\
& N_{3, \text { Wedge }}=\eta(1-\zeta) / 2 \\
& N_{4, \text { Wedge }}=(1-\xi-\eta)(1+\zeta) / 2 \\
& N_{5, \text { Wedge }}=\xi(1+\zeta) \\
& N_{6, \text { Wedge }}=\eta(1+\zeta) / 2,
\end{aligned}
$$

where $\zeta$ is the natural coordinate of the local element coordinate system in the $z$ direction. The limits of the natural coordinates for the three-dimensional wedge element are

$$
\begin{gathered}
0 \leq \xi, \eta \leq 1 \\
-1 \leq \zeta \leq 1 \\
\xi+\eta \leq 1 .
\end{gathered}
$$


Integrating the term

$$
\int_{\Omega^{(e)}}\left(\frac{\partial \dot{q}_{x}}{\partial x}+\frac{\partial \dot{q}_{y}}{\partial y}+\frac{\partial \dot{q}_{z}}{\partial z}\right) N_{i} d \Omega
$$

in Eq. (5) by Gauss's theorem introduces surface integrals of the heat flow across the element boundary. After this integration Eq. (5) becomes

$$
\begin{aligned}
\int_{\Omega^{(e)}} \rho c_{p} \frac{\partial T}{\partial t} N_{i} d \Omega-\int_{\Omega^{(e)}}\left[\frac{\partial N_{i}}{\partial x} \frac{\partial N_{i}}{\partial y} \frac{\partial N_{i}}{\partial z}\right]\left\{\begin{array}{c}
\dot{q}_{x} \\
\dot{q}_{y} \\
\dot{q}_{y}
\end{array}\right\} d \Omega \\
=\int_{\Omega^{(e)}} Q N_{i} d \Omega-\int_{\Gamma^{(e)}}(\dot{q} \cdot \hat{n}) N_{i} d \Gamma, \quad i=1,2, \ldots, D,
\end{aligned}
$$

where $\hat{n}$ is the unit normal to the surface, $\Gamma^{(e)}$ is the element surface boundary, and $D$ is the number of degrees of freedom in the element. The surface integral in Eq. (9) is now written as the sum of the integral over the portion of the surface with specified temperature boundary conditions, $S_{1}$, specified heat flow boundary conditions, $S_{2}$, convective heat exchange boundary conditions, $S_{3}$, and radiation heat exchange boundary conditions, $S_{4}$, leading to

$$
\begin{aligned}
\int_{\Omega^{(e)}} \rho c_{p} & \frac{\partial T}{\partial t} N_{i} d \Omega-\int_{\Omega^{(e)}}\left[\frac{\partial N_{i}}{\partial x} \frac{\partial N_{i}}{\partial y} \frac{\partial N_{i}}{\partial z}\right]\left\{\begin{array}{c}
\dot{q}_{x} \\
\dot{q}_{y} \\
\dot{q}_{y}
\end{array}\right\} d \Omega \\
= & \int_{\Omega^{(e)}} Q N_{i} d \Omega-\int_{S_{1}}(\dot{q} \cdot \hat{n}) N_{i} d \Gamma+\int_{S_{2}} \dot{q}_{a e r o} N_{i} d \Gamma-\int_{S_{3}} h\left(T_{w}-T_{e}\right) N_{i} d \Gamma \\
& -\int_{S_{4}}\left(\sigma \epsilon T_{w}^{4}-\alpha_{s} \dot{q}_{r}\right) N_{i} d \Gamma, \quad i=1,2, \ldots, D
\end{aligned}
$$

where $\dot{q}_{\text {aero }}$ is the heat flow rate per unit area due to aerodynamic heating(positive into the surface), $h$ is the convective heat transfer coefficient, $T_{w}$ is the wall temperature, $\sigma$ is the Stefan-Boltzman constant, $\epsilon$ is the surface emissivity, $\alpha_{s}$ is the surface absorptivity, and $\dot{q}_{r}$ is the incident radiant heat flow per unit area. We now express the temperatures and temperature gradients in terms of the shape functions and nodal values of temperature using

$$
\begin{aligned}
\mathcal{T}^{(e)}(x, y, z, t) & =N(x, y, z) T(t) \\
\frac{\partial \mathcal{T}^{(e)}(x, y, z, t)}{\partial x_{i}} & =B(x, y, z) T(t)
\end{aligned}
$$

where $B$ is the shape function derivative matrix, $\mathcal{T}^{(e)}$ gives the spatial distribution of the temperature over the domain of the element and $x_{i}$ indicates the three cartesian coordinate directions. The resulting system of equations for the finite element formulation then becomes

$$
M \dot{T}+K T=F_{Q}+F_{T}+F_{q}+F_{h}+F_{\sigma}
$$

where $M$ is the thermal capacitance matrix and $K$ is the thermal conductivity matrix. The vectors $F_{Q}, F_{T}$, $F_{q}, F_{h}$, and $F_{\sigma}$ are the thermal load vectors due to internal heat generation, specified nodal temperatures, specified surface heating, surface convection, and thermal radiation, respectively. The full-order system is solved using the same Crank-Nicolson scheme that is used to solve the reduced-order system and is described in the next section.

For the aerothermoelastic problem under consideration, the boundary conditions consist of only aerodynamic heating and thermal radiation. For the loads due to aerodynamic heating, a separate reduced-order modeling technique (to be described in a subsequent section) is used and thus these loads are treated as a specified surface heating condition for the purposes of the heat transfer model. Therefore, only the $F_{q}$ and $F_{\sigma}$ terms involving $S_{2}$ and $S_{4}$ on the right-hand side in Eq. (10), are included in the formulation. The radiation boundary condition is modeled by considering the outer surface of the structure to be gray and diffuse. To correctly model the radiation between the surface of the structure and the atmosphere, it must be taken into account that the gas molecules and suspended particles in the atmosphere emit and absorb 
radiation. ${ }^{38}$ Though atmospheric emission is far from resembling that of a blackbody, it is common to treat the atmosphere as a blackbody at a fictitious temperature, $T_{s k y}$, that emits an equivalent amount of radiation. ${ }^{38}$ While the value of $T_{s k y}$ varies depending on atmospheric conditions, we will take $T_{s k y}$ to be the atmospheric temperature, $T_{\infty}$, corresponding to the given flight altitude. The atmospheric irradation on a surface due to the atmosphere, $G_{s k y}$ can be expressed as $^{38}$

$$
G_{s k y}=\sigma T_{s k y}^{4} .
$$

The net radiation heat transfer to a surface exposed to solar and atmospheric radiation, $\dot{q}_{n e t, \text { rad }}$, is then given by

$$
\dot{q}_{\text {net }, \text { rad }}=\alpha_{s} G_{\text {solar }}+\alpha_{s} \sigma T_{s k y}^{4}-\epsilon \sigma T_{w}^{4},
$$

where $G_{\text {solar }}$ is the irradiation on the surface due to solar energy. In this work, solar irradiation will be neglected as the solar energy reaching the earth's surface on a clear day, about $950 \mathrm{~W} / \mathrm{m}^{2}$, will be much less than the incoming heat flux due to aerodynamic heating. Kirchoff's identity, given by ${ }^{39}$

$$
\alpha_{s}=\epsilon,
$$

will be employed to approximate the surface absorptivity and the thermal load vector in physical space, $F$, becomes

$$
F=F_{q}+F_{\sigma}=\int_{S}\left[\dot{q}_{a e r o}+\sigma \epsilon\left(T_{s k y}^{4}-T_{w}^{4}\right)\right] N_{i} d \Gamma,
$$

where the subscript on $S$ is dropped because both specified heat flux and radiation boundary conditions exist on the entire outer surface of the structure. The element load vector for the outer surface of the wedge element is calculated by integrating the shape functions for the two-dimensional triangular element, $N_{i, T r i}$, over the domain of the triangular outer surface of the element, i.e.,

$$
\begin{aligned}
& F_{1}^{(e)}=\dot{q}_{n e t} \int_{\Gamma^{(e)}} N_{1, T r i} d \Gamma^{(e)}=\dot{q}_{n e t} \int_{0}^{1} \int_{0}^{1-\xi} \xi|J| d \eta d \xi=\frac{\Delta}{3} \dot{q}_{n e t} \\
& F_{2}^{(e)}=\dot{q}_{n e t} \int_{\Gamma^{(e)}} N_{2, T r i} d \Gamma^{(e)}=\dot{q}_{n e t} \int_{0}^{1} \int_{0}^{1-\xi} \eta|J| d \eta d \xi=\frac{\Delta}{3} \dot{q}_{n e t} \\
& F_{3}^{(e)}=\dot{q}_{n e t} \int_{\Gamma^{(e)}} N_{3, T r i} d \Gamma^{(e)}=\dot{q}_{n e t} \int_{0}^{1} \int_{0}^{1-\xi}(1-\xi-\eta)|J| d \eta d \xi=\frac{\Delta}{3} \dot{q}_{n e t},
\end{aligned}
$$

where,

$$
\dot{q}_{n e t}=\dot{q}_{a e r o}+\sigma \epsilon\left(T_{s k y}^{4}-T_{w}^{4}\right)
$$

was taken out of the integral because it is assumed constant over each element, $|J|$ is the determinant of the finite element Jacobian matrix which is equal to twice the area of the outer surface of the solid element, $\Delta{ }^{36}$

\section{II.B. Reduced-Order Thermal Solution: Proper Orthogonal Decomposition}

\section{II.B.1. Creation of POD Basis}

This work makes use of POD for reduced-order solution of the transient thermal problem. POD, also known as the Karhunen-Loève decomposition, principal components analysis, singular systems analysis, and singular value decomposition, is a modal technique in which empirical data is processed using statistical methods to obtain models which capture the dominant physics of a system using a finite number of modes. ${ }^{40}$ The fundamental basis for use of POD as a reduced order modeling method is its ability to represent highdimensional systems in a low-dimensional, approximate manner while still maintaining a high degree of accuracy. The choice of the POD basis as opposed to any other basis such as an eigenvector basis is due to its optimality condition of providing the most efficient way of capturing the dominant components of a system with a finite number of modes. ${ }^{41}$ In addition to heat transfer problems, the POD has been used in numerous applications such as turbulence, ${ }^{42}$ structural dynamics, ${ }^{43,44}$ aerodynamics, ${ }^{45}$ and control theory ${ }^{46}$ among others.

The method of snapshots ${ }^{47}$ is used for determination of the POD basis vectors. In this case, the snapshots are defined as vectors of nodal temperatures at various time instants and are computed from high-fidelity 
finite element analysis. The goal of the POD formulation is to express the vector of nodal temperatures, $T$, at any time instant as a linear combination of the basis, $\varphi(x, y, z)$, with coefficients $c(t)$, i.e.,

$$
\left\{\begin{array}{c}
T_{1} \\
\vdots \\
T_{s}
\end{array}\right\}=c_{1}(t)\left\{\begin{array}{c}
\varphi_{1}^{(1)} \\
\vdots \\
\varphi_{s}^{(1)}
\end{array}\right\}+c_{2}(t)\left\{\begin{array}{c}
\varphi_{1}^{(2)} \\
\vdots \\
\varphi_{s}^{(2)}
\end{array}\right\}+\cdots+c_{r}(t)\left\{\begin{array}{c}
\varphi_{1}^{(r)} \\
\vdots \\
\varphi_{s}^{(r)}
\end{array}\right\},
$$

where $s$ is the total number of degrees of freedom in the finite element model and $r$ is the total number of POD basis vectors retained after truncation. The basis is computed by first generating the snapshot matrix, $A$, given by,

$$
A=\left[\begin{array}{cccc}
T_{1}^{(1)} & T_{1}^{(2)} & \ldots & T_{1}^{(n)} \\
T_{2}^{(1)} & T_{2}^{(2)} & \ldots & T_{2}^{(n)} \\
\vdots & \vdots & \ddots & \vdots \\
T_{s}^{(1)} & T_{s}^{(2)} & \ldots & T_{s}^{(n)}
\end{array}\right]=\left[a_{1}, a_{2}, \ldots, a_{n}\right]
$$

where $T_{i}^{(j)}$ indicates the $i$-th entry of the $j$-th snapshot, $n$ is the number of snapshots taken and $a_{j}$ refers to column vector corresponding to the $j$-th snapshot. The correlation matrix, $C$, is then found, whose entries are the inner products of the corresponding snapshots, given by

$$
C_{i j}=\frac{1}{n} a_{i}^{T} a_{j} \text { or } C=\frac{1}{n} A^{T} A .
$$

Solution of the eigenvalue problem,

$$
C v_{i}=\lambda_{i} v_{i},
$$

yields the eigenvectors and eigenvalues of the correlation matrix where $v_{i}$ with $\left\|v_{i}\right\|=1$ indicates the $i$-th eigenvector of $C$ corresponding to the $i$-th largest eigenvalue of $C$. Note that the eigenvectors are arranged such that they correspond with eigenvalues that are sorted in decreasing magnitude so that the POD basis vectors will be sorted in order of decreasing energy. The eigenvalues, $\lambda$, and eigenvectors, $v$, of the correlation matrix are then used to generate the POD basis vectors, $\varphi$, which are expressed as a linear combination of the snapshots, i.e.,

$$
\varphi_{k}=\frac{1}{\sqrt{n \lambda_{k}}} \sum_{i=1}^{n} v_{i}^{(k)} a_{i}=\frac{1}{\sqrt{n \lambda_{k}}} A v_{k}
$$

where $v_{i}^{(k)}$ is the $i$-th entry of the $k$-th eigenvector. The POD modal matrix, $\Phi$, can then be assembled with the POD basis vectors, $\varphi_{i}$, stored as columns of the matrix. The POD basis is then truncated to a reduced set of POD vectors, $\bar{\Phi}$, thus leading to a reduction in the number of degrees of freedom in the problem. Note that both the full and truncated POD sets are orthogonal, i.e.

$$
\begin{gathered}
\Phi^{T} \Phi=I_{n} \\
\bar{\Phi}^{T} \bar{\Phi}=I_{r},
\end{gathered}
$$

where $I_{n}$ represents the identity matrix of dimension $n$ and $I_{r}$ refers the the identity matrix of dimension $r$.

The columns of the snapshot matrix can be expressed as linear combinations of the basis vectors with coefficients $\chi$ using the expression ${ }^{48}$

$$
A=\Phi \chi
$$

where $\chi$ is a square matrix of coefficients of the full set of POD basis vectors and can be calculated by making use of the orthogonality of the POD basis as

$$
\chi=\Phi^{T} A .
$$

As the basis will be truncated to reduce the problem, the snapshot matrix can at best be approximated via a linear combination of the truncated POD modal matrix, $\bar{\Phi}$, given by

$$
A \approx \bar{\Phi} \bar{\chi}
$$


where $\bar{\chi}$ is a square matrix of coefficients of the truncated set of POD basis vectors and can be calculated in a manner similar to that of Eq. (27) using

$$
\bar{\chi}=\bar{\Phi}^{T} A,
$$

where the orthogonality of $\bar{\Phi}$ is utilized. As the truncated basis can only approximate the snapshots, the goal is to find the optimal basis such that the approximated snapshot matrix, $\bar{\Phi} \bar{\chi}$, represents as closely as possible the actual snapshot matrix, $\Phi \chi$. Let $\Psi$ represent an arbitrary orthonormal $s \times n$ basis and $\bar{\Psi}$ represent the corresponding $s \times r$ truncated basis. Let the error incurred as a result of basis truncation, $\varepsilon$, be given by ${ }^{48,49}$

$$
\varepsilon=\|A-\bar{\Psi} \bar{\chi}\|^{2} .
$$

Using Eq. (29) in Eq. (30) and substituting the truncated arbitrary basis, $\bar{\Psi}$, for the truncated POD basis, $\bar{\Phi}$, the error expression becomes ${ }^{48}$

$$
\varepsilon=\left\|A-\bar{\Psi} \bar{\Psi}^{T} A\right\|^{2} .
$$

At this point, the quantity $\bar{\Psi} \bar{\Psi}^{T}$ is recognized as the orthogonal projector that projects onto the $r$-dimensional subspace spanned by the basis, $\bar{\Psi} \cdot{ }^{49,50}$ Thus, Eq. (31) can be written as

$$
\varepsilon=\left\|A-P_{\bar{\Psi}, r} A\right\|^{2},
$$

where $P_{\bar{\Psi}, r}$ is the orthogonal projector onto the $r$-dimensional subspace. The error due to basis truncation can then be interpreted as the amount by which the projection of the snapshots onto the truncated basis differs from the snapshots themselves. The objective is therefore to find an orthonormal basis such that for a specified error, $\varepsilon$, a minimum number of columns of the set of basis vectors must be retained. A supplementary condition following from Eq. (26) is that the basis is a linear combination of the snapshots as seen in Eq. (24). It has been shown ${ }^{41}$ that along with this supplementary condition, the minimum error in Eq. (32) occurs when the basis, $\bar{\Psi}$, is chosen to be the POD basis, $\bar{\Phi}$, as given in Eq. (24). Thus the POD basis is optimal with respect to any other linear modal representation in that the first $k$ POD modes contain more energy than the first $k$ modes of any other basis. ${ }^{40}$

An alternative method for calculating the POD basis involves the singular value decomposition (SVD). The full SVD of the $s \times n$ snapshot matrix, $A$, is given by ${ }^{51}$

$$
A=U \Sigma V^{T},
$$

where $U$ is a $s \times s$ orthogonal matrix, $V$ is an $n \times n$ orthogonal matrix, and $\Sigma$ is a $s \times n$ diagonal matrix with

$$
\sigma_{i j}=\left\{\begin{array}{ll}
0 & \text { for } i \neq j \\
\sigma_{i} \geq 0 & \text { for } i=j
\end{array} .\right.
$$

The diagonal entries of $\Sigma$ are known as the singular values of $A$ and are ordered in decreasing order such that $\sigma_{1} \geq \sigma_{2} \geq \ldots \geq 0$. The columns of $U$ and $V$ are the left and right singular vectors of $A$, respectively. The correspondence between the POD basis as derived using the eigenvalues of the correlation matrix, $\lambda_{i}$, and that derived using the SVD can be shown by relating $\lambda_{i}$ to $\sigma_{i}$. Performing the SVD of $C$ in terms of the SVD of $A$, we have

$$
C=\frac{1}{n} A^{T} A=\frac{1}{n}\left(U \Sigma V^{T}\right)^{T} U \Sigma V^{T}=\frac{1}{n} V \Sigma^{T} U^{T} U \Sigma V^{T} .
$$

Taking advantage of the orthogonality of $U$, Eq. (35) becomes

$$
C=\frac{1}{n} V\left(\Sigma^{T} \Sigma\right) V^{T}
$$

From Eq. (36) we can see that $V$ is the matrix of eigenvectors of $C$ and the singular values of $A$ are related to the eigenvalues of $C$ according to ${ }^{49}$

$$
\sigma_{i}^{2}=n \lambda_{i} .
$$

The POD basis vectors can be shown to correspond to the left singular vectors of the snapshot matrix ${ }^{49}$ if we re-write Eq. (24) in terms of the SVD of $A$, i.e.,

$$
\varphi_{k}=\frac{1}{\sqrt{n \lambda_{k}}} U \Sigma V^{T} v_{k}
$$


Due to orthogonality of $V$, Eq. (38) simplifies to

$$
\varphi_{k}=\frac{1}{\sqrt{n \lambda_{k}}} u_{k} \sigma_{k},
$$

where $u_{k}$ is the $k$-th left singular vector of $A$. Utilizing Eq. (37), we have $\varphi_{k}=u_{k}$. The connection between POD and SVD allows for justification of the claim of basis optimality due to the ability of the SVD to provide an optimal low-rank approximation to a matrix. Consider an approximation to $A$ written as a partial sum of rank-one matrices formed from the outer product of the left and right singular vectors with the singular value as the scalar coefficient, given by

$$
A=\sum_{j=1}^{r} \sigma_{j} u_{j} v_{j}^{T}
$$

where $r$ corresponds to the number of POD basis vectors retained after truncation. It can be shown that the $r$-th partial sum captures the maximum possible amount of energy of $A$, where energy is defined in either the 2-norm or Frobenius norm sense. ${ }^{50-52}$ Alternatively stated, no other rank $r$ matrix can be closer to $A$ in the 2-norm or in the Frobenius norm. To examine the connection between this optimality property of the SVD and the optimality of the POD basis, consider Eq. (30) written in terms of the truncated set of left singular vectors, $\bar{U}$, corresponding to the truncated set of POD basis vectors, $\bar{\Phi}$, given by

$$
\varepsilon=\left\|A-\bar{U} \bar{U}^{T} A\right\|^{2} .
$$

Now, expressing $A$ in terms of its full SVD leads to,

$$
\varepsilon=\left\|U \Sigma V^{T}-\bar{U} \bar{U}^{T} U \Sigma V^{T}\right\|^{2} .
$$

At this point, the quantity $\bar{U} \bar{U}^{T} U$ is recognized as the projection of $U$ onto the space spanned by $\bar{U}$ and performs the action of zeroing the columns of $U$ that correspond to the excluded POD basis vectors. Thus, Eq. (42) becomes

$$
\varepsilon=\left\|\sum_{j=1}^{n} \sigma_{j} u_{j} v_{j}^{T}-\sum_{j=1}^{r} \sigma_{j} u_{j} v_{j}^{T}\right\|^{2},
$$

and the optimality of the POD basis is demonstrated due to the optimality property of the SVD described above. The absolute error associated with the $r$-dimensional POD subspace is associated with the eigenvalues of the correlation matrix, $C$, and the singular values of the snapshot matrix, $A$, and is given by ${ }^{49}$

$$
\varepsilon=\sum_{j=r+1}^{n} \sigma_{j}^{2}=n \sum_{j=r+1}^{n} \lambda_{j},
$$

where $\varepsilon$ is defined in the Frobenius norm sense. A relative error tolerance, $\varepsilon_{r e l}$, can be defined such that if the relative error is to be less than an error tolerance, $\varepsilon_{T}$, i.e.,

$$
\frac{\varepsilon}{\|A\|^{2}}=\varepsilon_{r e l} \leq \varepsilon_{T}
$$

the number of basis vectors retained, $r$, should be the smallest integer that

$$
\frac{\sum_{j=1}^{r} \sigma_{j}^{2}}{\sum_{j=1}^{n} \sigma_{j}^{2}}=\frac{\sum_{j=1}^{r} \lambda_{j}}{\sum_{j=1}^{n} \lambda_{j}} \geq 1-\varepsilon_{T} .
$$

Note that the left-hand side of Eq. (46) can be interpreted as the energy of the included basis vectors relative to the energy of the full set of basis vectors. Furthermore, $\varepsilon_{r e l}$ can be interpreted as the energy of the excluded basis vectors relative to the energy of the full set of basis vectors, given by

$$
\varepsilon_{r e l}=\frac{\varepsilon}{\|A\|^{2}}=\frac{\sum_{j=r+1}^{n} \sigma_{j}^{2}}{\sum_{j=1}^{n} \sigma_{j}^{2}}=\frac{\sum_{j=r+1}^{n} \lambda_{j}}{\sum_{j=1}^{n} \lambda_{j}} .
$$

Thus, the magnitude of each of the eigenvalues of the correlation matrix can be used in determining the number of POD basis vectors that can be removed from the set. 


\section{II.B.2. Solution of System for Modal Coordinates}

While a recent effort described a method for decoupling the reduced-order system of ordinary differential equations and solving for the modal coordinates analytically, ${ }^{48}$ in this work we will solve for the modal coordinates numerically because we will only have a discrete representation of the thermal loads as opposed to a continuous one. Numerical solutions of the coupled thermal problem are expected to be more useful than decoupled analytical solution within hypersonic vehicle aerothermoelastic simulation frameworks. This is due to the fact that the heat flux depends on various factors such as deformation and aerodynamic flow properties that are not known ahead of time. The thermal load vector must therefore be sampled at specific time instants. Note that one could decouple the equations and integrate the thermal loads numerically, however at that point, the ability to obtain a purely analytical solution is lost. Additionally, solving the coupled system directly avoids the need to solve an eigenvalue problem to diagonalize the equations. This is especially important for nonlinear problems in which the thermal capacitance matrix and thermal conductivity matrix change with temperature and an eigenvalue problem would need to be solved at every time step if the equations were to be decoupled.

For the numerical solution, the transient equations are solved using a numerical time-marching algorithm. $^{35,53}$ For nonlinear cases in which the system matrices or load vector depend on the temperatures at the current time step, Newton-Raphson iterations can be employed at each time step. To begin the formulation, consider the full-order system of first order coupled ordinary differential equations governing transient heat transfer as derived in Eq. (13),

$$
M \dot{T}+K T=F(t),
$$

The first step is to project the full-order system onto the truncated POD basis and transform from physical space to modal space using

$$
\bar{\Phi}^{T} M \bar{\Phi} \dot{c}+\bar{\Phi}^{T} K \bar{\Phi} c=\bar{\Phi}^{T} F
$$

The generalized thermal capacitance matrix, $m$, generalized thermal conductivity matrix, $k$, and generalized load vector, $f$, are then identified such that

$$
\begin{aligned}
m & =\bar{\Phi}^{T} M \bar{\Phi} \\
k & =\bar{\Phi}^{T} K \bar{\Phi} \\
f & =\bar{\Phi}^{T} F .
\end{aligned}
$$

Note that because the POD basis vectors are not eigenvectors of the generalized eigenvalue problem, $m$ and $k$ will still not be diagonal at this stage. For the numerical solution of the equations, we will not perform the additional step to solve the eigenvalue problem and decouple the equations. Rather, the reduced-order coupled system will be integrated numerically at this stage. We denote a time instant in the response of the system by $t_{n}$ such that the time instant at the next time step is given by $t_{n+1}=t_{n}+\Delta t_{H T}$. A parameter $\theta$ is introduced to represent the response of the system at an intermediate time, $t_{\theta}$, such that $t_{\theta}=t_{n}+\theta \Delta t_{H T}$, where $0 \leq \theta \leq 1$. Expressing Eq. (48) at time $t_{\theta}$ in modal space, we have,

$$
m \dot{c}_{\theta}+k c_{\theta}=f\left(t_{\theta}\right)
$$

where the subscript $\theta$ indicates the vector of unknowns, at time instant $t_{\theta}$. We now introduce approximations to $c$, its time-derivative, and $f$ at $t_{\theta}$ given by

$$
\begin{aligned}
\dot{c}_{\theta} & =\frac{c_{n+1}-c_{n}}{\Delta t_{H T}} \\
c_{\theta} & =(1-\theta) c_{n}+\theta c_{n+1} \\
f\left(t_{\theta}\right) & =(1-\theta) f_{n}+\theta f_{n+1} .
\end{aligned}
$$

Substituting Eqs. (52) into Eq. (51) leads to the recurrence relation given by ${ }^{35}$

$$
\left[\theta k+\frac{1}{\Delta t_{H T}} m\right] c_{n+1}=\left[-(1-\theta) k+\frac{1}{\Delta t_{H T}} m\right] c_{n}+(1-\theta) f_{n}+\theta f_{n+1},
$$

where $c_{n+1}$ is the unknown to be found and $c_{n}$ is the solution from the previous time step. The recurrence relation given in Eq. (53) is of the form

$$
L c_{n+1}=b_{n+1},
$$


where

$$
\begin{aligned}
L & =\theta k+\frac{1}{\Delta t_{H T}} m \\
b_{n+1} & =\left[-(1-\theta) k+\frac{1}{\Delta t_{H T}} m\right] c_{n}+(1-\theta) f_{n}+\theta f_{n+1} .
\end{aligned}
$$

Equation (53) represents a family of recurrence relations in which the particular numerical scheme depends on the chosen value of $\theta$. For $\theta=0, \frac{1}{2}, \frac{2}{3}$, and 1, the algorithm represents the forward Euler, Crank-Nicolson, Galerkin, and backward Euler method, respectively. Additionally, if $\theta=0$, the algorithm is explicit, while for $\theta>0$, the algorithm is implicit. The requirements for convergence of the selected numerical scheme are given by the Lax Equivalence Theorem which states that for a well-posed initial value problem and a consistent discretization scheme, stability is the necessary and sufficient condition for convergence. ${ }^{51,54}$ The family of $\theta$ algorithms described above is unconditionally stable in the linear case for $\theta \geq \frac{1}{2}$ while an extension for nonlinear systems ${ }^{55}$ was also shown to be unconditionally stable for $\theta \geq \frac{1}{2}$. For $\theta<\frac{1}{2}$, the algorithm is conditionally stable and the eigenvalue stability region is such that the critical time step is given by

$$
\Delta t_{c r}=\frac{2}{1-2 \theta} \frac{1}{\gamma_{m}}
$$

where $\gamma_{m}$ is the largest system eigenvalue. For this work, the Crank-Nicolson scheme corresponding to $\theta=\frac{1}{2}$ is used due to its second-order accuracy.

Since the equations are solved in modal space and the initial temperatures are known in physical space, the initial values of the modal coordinates must first be calculated before time-marching can proceed. Using the transformation between physical space and modal space, we begin by expressing the vector of initial temperatures in physical space as can be expressed as

$$
T_{0}=\bar{\Phi} c_{0}
$$

where $c_{0}$ is the vector of initial values of the modal coordinates. Expanding this expression in terms of the individual POD vectors, Eq. (57) becomes

$$
T_{0}=c_{0}^{(1)} \varphi_{1}+c_{0}^{(2)} \varphi_{2}+\cdots+c_{0}^{(r)} \varphi_{r},
$$

where $\varphi_{i}$ indicates the $i$-th basis vector and $c_{0}^{(i)}$ refers to the corresponding $i$-th modal coordinate at time $t_{0}$. To find the initial value of the $i$-th modal coordinate, Eq. (58) is premultiplied by the transpose of the $i$-th basis vector, $\varphi_{i}^{T}$, to obtain

$$
\varphi_{i}^{T} T_{0}=c_{0}^{(1)} \varphi_{i}^{T} \varphi_{1}+c_{0}^{(2)} \varphi_{i}^{T} \varphi_{2}+\cdots+c_{0}^{(r)} \varphi_{i}^{T} \varphi_{r}
$$

Recall that the basis vectors are an orthonormal set such that

$$
\varphi_{i}^{T} \varphi_{j}=\delta_{i j},
$$

where $\delta_{i j}$ is the Kronecker delta. Thus, the right-hand side of Eq. (59) reduces to $c_{0}^{(i)}$ and the left-hand side gives its value. By premultiplying Eq. (58) by each of the basis vectors, the complete vector of initial modal coordinates can be found and time-marching of the system can proceed.

\section{Transient Structural Dynamic Response Modeling}

\section{III.A. High-Fidelity Structural Dynamic Response Solution}

The full-order system of structural dynamic equations of motion in physical space is given by

$$
M_{s} \ddot{x}+K_{s}^{*}(T) x=F_{s}(t),
$$

where $M_{s}$ is the mass matrix, $F_{s}$ is the load vector, and $x$ are the physical degrees of freedom. The modified stiffness matrix, $K_{s}^{*}$, is given by

$$
K_{s}^{*}(T) \equiv K_{s}(T)+K_{G}(T),
$$


where $K_{s}(T)$ is the conventional stiffness matrix that varies due to the temperature-dependence of the material properties and $K_{G}(T)$ is the geometric stiffness matrix resulting from thermal stresses. Thermal loading which puts the structure in tension results in an increase in stiffness while that which puts the structure in compression results in a decrease in stiffness. For the high-fidelity solution of the equations of motion, a direct transient response solution (Sol 109) is used within the finite element code Nastran. The numerical integration method is similar to the Newmark- $\beta$ method except that the load vector is averaged over three time instants and the stiffness matrix is modified such that the dynamic equation of motion reduces to a static solution if no inertial effects or damping exists. ${ }^{56}$ The scheme uses a central finite difference representation for the velocity and acceleration at discrete times, given by ${ }^{56}$

$$
\begin{aligned}
& \dot{x}_{n}=\frac{1}{2 \Delta t_{A E}}\left(x_{n+1}-x_{n-1}\right) \\
& \ddot{x}_{n}=\frac{1}{\Delta t_{A E}^{2}}\left(x_{n+1}-2 x_{n}+x_{n-1}\right),
\end{aligned}
$$

where $n$ refers to the time level. The initial conditions, $x_{0}$ and $\dot{x}_{0}$, are used to generate the vectors $x_{n-1}$, $F_{s, n-1}$, and $F_{s, n}$ for the initial time step, $n=0$, using

$$
\begin{aligned}
\dot{x}_{-1} & =x_{0}-\dot{x}_{0} \Delta t_{A E} \\
F_{s,-1} & =K_{s} x_{-1} \\
F_{s, 0} & =K_{s} x_{0} .
\end{aligned}
$$

Note that this formulation assumes that the inital acceleration for all points is zero (initial velocity is constant). Substituting the finite difference approximations of the velocity and accelerations, Eqs. (63), into the equations of motion, Eq. (61) and averaging the applied loads over three adjacent time instants, the equations of motion are re-written as

$$
H_{1} x_{n+1}=H_{2}+H_{3} x_{n}+H_{4} x_{n-1}
$$

where,

$$
\begin{aligned}
H_{1} & =\frac{1}{\Delta t_{A E}^{2}} M_{s}+\frac{1}{3} K_{s} \\
H_{2} & =\frac{1}{3}\left(F_{s, n+1}+F_{s, n}+F_{s, n-1}\right) \\
H_{3} & =\frac{2}{\Delta t_{A E}^{2}} M_{s}-\frac{1}{3} K_{s} \\
H_{4} & =\frac{-1}{\Delta t_{A E}^{2}} M_{s}-\frac{1}{3} K_{s} .
\end{aligned}
$$

The displacement vector at the next time step, $x_{n+1}$, is obtained by decomposing $H_{1}$ and applying it to the right-hand side of Eq. (65).

\section{III.B. Reduced-Order Modal Basis Solution for Structural Dynamic Response}

While the full-order system of structural dynamic equations of motion is used for comparison purposes, its solution within the aerothermoelastic framework presented here is not suitable for control system design and simulation purposes. The problem of solving for the structural dynamic response of hypersonic vehicle structures within a control design and evaluation framework is complicated by various factors. Due to the large number of degrees of freedom involved in a traditional finite element solution, steps must be taken to reduce the order of the structural dynamics system of equations. A common approach is to employ a normal modal transformation in which the structural displacements are written as a linear combination of a small number of basis vectors which are the free vibration mode shapes of the structure. However, this approach cannot be applied directly for hypersonic vehicle applications as the mode shapes change over time due to modification of the stiffness from geometric stiffness and material degradation effects. The approach taken in this work is to first perform an off-line calculation and select a reduced number of Ritz modes based on the free vibration modes of the structure at a reference temperature distribution. These Ritz modes are then 
used as the modal basis for solution of the structural response throughout the simulation. This procedure is applicable as the Ritz modes need only to satisfy the geometric boundary conditions, ${ }^{57}$ which will always be the case regardless of the stiffness distribution. The modal matrix containing the structural reference modes, $\Phi_{r e f}$, will not be updated throughout the simulation, thus preventing the need to solve an eigenvalue problem of the full system during the course of the simulation. Though the reference modes will not be updated throughout the simulation, the modified stiffness matrix will be updated each time the structural dynamic response is calculated to account for the dependence on temperature. Updating of the conventional stiffness matrix is performed using the temperature-dependence of the material properties of the various materials. The geometric stiffness matrix is updated by solving a static finite element problem based on the thermal loads from temperatures at the current time step and the material coefficients of thermal expansion to calculate the internal loads.

The reduced-order system is obtained by first expressing the physical degrees of freedom as a linear combination of the reference free vibration modes such that

$$
x=\Phi_{r e f} d,
$$

where $d$ represents the modal coordinates of the reference modes which are stored as columns of the modal matrix, $\Phi_{r e f}$. Note that since the number of reference modes used in the modal expansion is much less than the number of physical degrees of freedom in the model, the computational cost of the numerical solution of the system is relatively inexpensive. Once the modified stiffness matrix is known at the current time instant, the system is reduced by substituting Eq. (67) into Eq. (61) and pre-multiplying the system by $\Phi_{\text {ref }}^{T}$, i.e.

$$
\Phi_{r e f}^{T} M_{s} \Phi_{r e f} \ddot{d}+\Phi_{r e f}^{T} K_{s}^{*}(T) \Phi_{r e f} d=\Phi_{r e f}^{T} F_{s}(t) .
$$

The generalized mass matrix, $m_{s}$, generalized stiffness matrix, $k_{s}$, and generalized load vector, $f_{s}$ are then given by

$$
\begin{aligned}
m_{s} & =\Phi_{r e f}^{T} M_{s} \Phi_{r e f} \\
k_{s} & =\Phi_{r e f}^{T} K_{s} \Phi_{r e f} \\
f_{s} & =\Phi_{r e f}^{T} F_{s} .
\end{aligned}
$$

As the mass of the structure is taken to be constant in this work, the reference modes will be orthogonal with respect to the mass matrix and the generalized mass matrix, $m_{s}$, will reduce to the identity matrix. Since the modified stiffness matrix will be continuously changing, we have no guarantee of orthogonality of the reference modes with respect to stiffness, and the equations will be coupled. As such, the reduced-order system of equations in modal space is integrated numerically to calculate the vector of modal coordinates at each time instant. As the high-fidelity structural dynamic response solution is treated as the truth model, the numerical integration scheme used for the high-fidelity model given in the previous section is implemented for integration of the reduced-order system to eliminate any discrepancies in the response due to differences in numerical integration schemes.

\section{Reduced-Order Aerothermal Modeling}

In order to compute the aerodynamic heat flux, a reduced-order aerothermodynamic model (ROAM) is generated from CFD solutions to the Navier-Stokes equations using kriging. ${ }^{32,34,58-63}$ A fundamental assumption of kriging is that two sample points that are close together in the parameter space will have similar errors. This assumption of correlated errors is appropriate since no sources of random error or "noise" exist in deterministic computer simulations. ${ }^{63}$ Kriging is a useful method for replacing expensive computer models (i.e., CFD) with computationally efficient approximations, or surrogates, of nonlinear functions. ${ }^{62,64,65}$ Typical surrogate prediction times are on the order of a fraction of a second, whereas a single CFD computation may take on the order of minutes to hours. ${ }^{34}$ Furthermore, kriging does not require a priori assumptions on the form of the full-order function that is to be approximated, unlike polynomial response surfaces.

A kriging interpolation is generated from training data, or snapshots, of the full order simulation of the computer model. The kriging approximation of the function of interest is characterized by local deviations, $C(x)$, from a global approximation, $R(x)$, as defined by ${ }^{59}$ 


$$
y(d)=R(d, X)+C(d, X),
$$

where $y(d)$ is the kriging prediction at a desired point, $d$, and $X$ are the sample points of the training data. For this study, the kriging surrogates are computed using the Design and Analysis of Computer Experiments $(\mathrm{DACE})^{58}$ toolbox in Matlabß. Typically, $R(d, X)$ is a polynomial regression function, which is assumed to be either constant, linear, or quadratic, and the constants of the polynomials are generally determined in a least-squares sense. The quantity $C(d, X)$ provides the local deviations by means of a correlation function, defined by the user. In this study the three aforementioned polynomial regression functions, as well as a Gaussian correlation function ${ }^{32,58,59,62,63}$ will be investigated in order to identify the most accurate kriging surrogate for this study. Note, steady-state CFD solutions are used for the kriging training data, due to large differences in the characteristic time scale. ${ }^{31}$ Thus, the aerothermal and aeroelastic systems are loosely coupled in time.

For the current study, the ROAM is constructed to compute the aerodynamic heat flux on the control surface as a function of: 1) freestream Mach number, 2) angle of attack, 3) altitude, 4) surface deformation, and 5) surface temperature. Both the structural deformation and surface temperature are represented consistent with the modal bases determined for the reduced-order thermal and structural models, i.e.

$$
\begin{gathered}
w(x, y)=d_{1} \Phi_{r e f, 1}(x, y)+d_{2} \Phi_{r e f, 2}(x, y)+\cdots+d_{n_{s}} \Phi_{r e f, n_{s}}(x, y) \\
T_{w}(x, y)=c_{1}\left\{\begin{array}{c}
\varphi_{1}^{(1)} \\
\vdots \\
\varphi_{k}^{(1)}
\end{array}\right\}+c_{2}\left\{\begin{array}{c}
\varphi_{1}^{(2)} \\
\vdots \\
\varphi_{k}^{(2)}
\end{array}\right\}+\cdots+c_{n_{t}}\left\{\begin{array}{c}
\varphi_{1}^{\left(n_{t}\right)} \\
\vdots \\
\varphi_{k}^{\left(n_{t}\right)}
\end{array}\right\}
\end{gathered}
$$

where $\left\{\varphi_{1}^{(i)} \cdots \varphi_{k}^{(i)}\right\}^{T}$ represent the surface nodes of the $i$-th POD mode of the thermal problem. Therefore, the ROAM will include two trajectory parameters (Mach number and altitude), one control input parameter (angle of attack), $n_{s}$ structural parameters $\left(d_{i}\right)$, and $n_{t}$ thermal parameters $\left(c_{i}\right)$. The bounds for these parameters are shown in Table 1,

Table 1. Bounds of parameters used to construct the aerodynamic heating ROAM.

\begin{aligned} \hline \hline 5.0 & $\leq M_{\infty} \leq 10.0 \\ -5.0^{\circ} & \leq \alpha \leq 5.0^{\circ} \\ 25.0 \mathrm{~km} & \leq A l t . \leq 45.0 \mathrm{~km} \\ -10 \% & \leq w / L \leq 10 \% \\ T_{\infty} \leq T_{w} \leq 1500 \mathrm{~K} & \leq\end{aligned}$

where $w$ is the nodal deflection of the wing in the $\mathrm{z}$ direction, $L$ is the distance from the attachment point (located at the root mid-chord) to each node, and $T_{\infty}$ is the freestream temperature at the altitude of the sample point. These bounds are selected based on expected operating conditions, linearity of deformation, and maximum temperature limits of the heat shield. A flowchart of the ROAM construction is provided in Fig. 4.

Given the control surface configuration and the bounds on the parameter space defined in Table 1, ROAM construction begins with generation of training data. First, Latin Hypercube Sampling (LHS) is used to generate a random, yet uniformly distributed sampling of the selected parameter space. The sample points are then passed to the CFD solver and the aerodynamic heating solutions for the flow field are collected; $n_{e}$ snapshot solutions are saved for evaluation of the ROAM accuracy, while $n_{k}$ snapshot solutions are utilized in conjunction with the Design and Analysis of Computer Experiments (DACE) toolbox ${ }^{58}$ in Matlabß). The DACE toolbox is used to construct the kriging surrogate models. This toolbox allows for the rapid interchange of different polynomial regression functions, $R(x)$, and correlation functions, $C(x)$, in order to identify the most accurate kriging surrogate method. The goal is for the kriging surrogate to maintain less than $5 \%$ maximum error relative to the full-order evaluation snapshots. If the desired accuracy of the surrogate is not met, more sample points can be added to the ROAM process. 


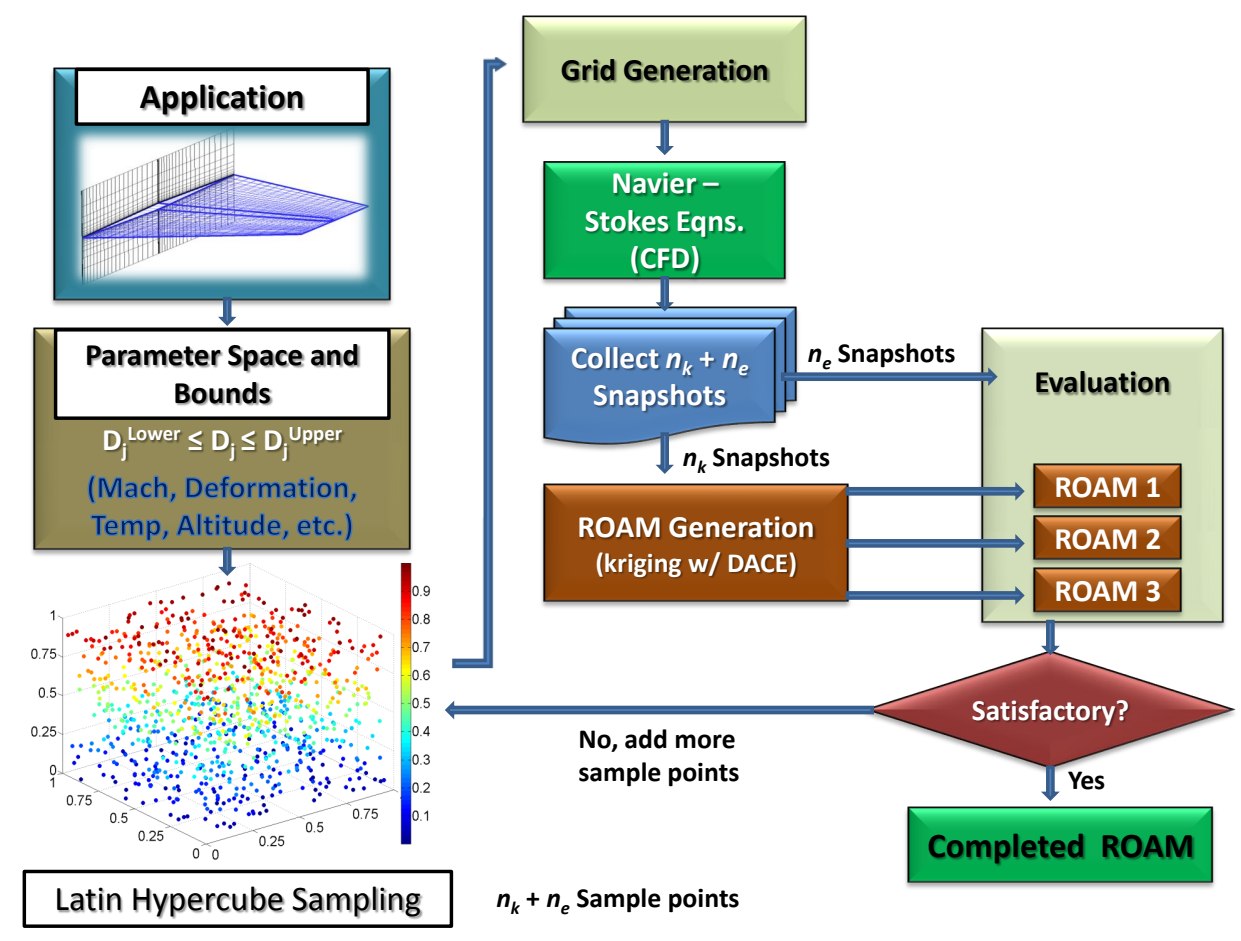

Figure 4. Schematic of ROAM framework.

\section{Unsteady Aerodynamic Modeling}

Piston theory is used for calculation of the aerodynamic pressures acting on the outer surface of the structure. This technique provides a closed form expression which relates the local pressure resulting from a body's motion to the normal component of the fluid velocity at the location of interest. Early development of piston theory was performed by Lighthill ${ }^{66}$ who discussed its application to oscillating airfoils. Ashley and Zartarian ${ }^{67}$ further discussed the theory and applied it to a variety of aeroelastic problems. The fundamental underpinning of this aerodynamic theory is that in a two-dimensional inviscid flow, a perpendicular column of fluid stays intact as it passes over the surface of a structure. ${ }^{10}$ Thus, the unsteady pressure is calculated at a specific location as if it were the face of a piston moving into a one-dimensional channel. The pressure on the face of a piston moving in a channel of perfect gas with velocity $V_{n}$ is given by ${ }^{67}$

$$
\frac{p}{p_{\infty}}=\left(1+\frac{\gamma-1}{2} \frac{V_{n}}{a_{\infty}}\right)^{\frac{2 \gamma}{\gamma-1}}
$$

where $p$ is the surface pressure, $p_{\infty}$ is the freestream pressure, $\gamma$ is the ratio of specific heats, $V_{n}$ is the velocity of the surface normal to the flow, and $a_{\infty}$ is the freestream speed of sound. Due to the hypersonic flow regime considered in this work, third-order piston theory is utilized. ${ }^{13}$ The pressure coefficient based on third order expansion of Eq. (73) is given by ${ }^{4}$

$$
C_{p}=\frac{2}{M_{\infty}^{2}}\left[\frac{V_{n}}{a_{\infty}}+\frac{(\gamma+1)}{4}\left(\frac{V_{n}}{a_{\infty}}\right)^{2}+\frac{(\gamma+1)}{12}\left(\frac{V_{n}}{a_{\infty}}\right)^{3}\right],
$$

where $M_{\infty}$ is the freestream Mach number. The pressure coefficient for compressible flow is given by ${ }^{68}$

$$
C_{p}=\frac{2}{\gamma M_{\infty}^{2}}\left(\frac{p}{p_{\infty}}-1\right) .
$$

Using Eq. (75) in Eq. (74), one obtains ${ }^{69}$

$$
p-p_{\infty}=\gamma p_{\infty}\left[\frac{V_{n}}{a_{\infty}}+\frac{(\gamma+1)}{4}\left(\frac{V_{n}}{a_{\infty}}\right)^{2}+\frac{(\gamma+1)}{12}\left(\frac{V_{n}}{a_{\infty}}\right)^{3}\right] .
$$


Note that $V_{n}$ is given in terms of the freestream velocity, $V_{\infty}$, and the position of the outer surface of the structure, $Z(x, y, t)$, by

$$
V_{n}=\frac{\partial Z(x, y, t)}{\partial t}+V_{\infty}\left[\frac{\partial Z(x, y, t)}{\partial x}+\alpha\right]
$$

where $x$ is the freestream direction, and $Z(x, y, t)$ is the position of the surface of the structure. Note that $Z(x, y, t)$ is a function of the elastic deformation of the structure and the geometry of the outer mold line and is given by

$$
Z(x, y, t)=w_{d}(x, y, z, t)+Z_{s t r}(x, y)
$$

where $w_{d}$ is the displacement in the $z$ direction normal to the flow direction and $Z_{s t r}(x, y)$ is a function describing the geometry of the outer mold line of the structure. Calculation of the spatial and temporal partial derivatives in Eq. (77) are performed using finite difference approximations at the nodal locations of the finite element grid.

One restriction on the use of piston theory is that the magnitude of the piston velocity, given by $V_{n}$ must never exceed the speed of sound in the undisturbed fluid. ${ }^{66}$ As the control surface is likely to undergo relatively large changes in angle of attack, it is likely that this assumption would be violated if piston theory was used in isolation. To reduce $V_{n}$ and decrease the likelihood that this assumption is violated, the flow is turned through the rigid angle of attack at the leading edge using the oblique shock and PrandtlMeyer expansion theory relations described in a previous work. ${ }^{29}$ Use of these relations allows for $\alpha$ to be removed from the piston velocity expression given in Eq. (77) and therefore reduces the slope used in the calculation of the piston velocity. Thus, the freestream velocity, $V_{\infty}$, in Eq. (77) now becomes the post-shock or post-expansion velocity corresponding to the flow over the top or bottom surface as calculated using the shock/expansion relations.

\section{Influence of Flexibility on Aerodynamic Performance and Control Input}

A goal of this work is to assess the aerothermoelastic effects on the vehicle control effectors. These effects ultimately result in changing aerodynamic forces and moments on the vehicle over time, which will in turn affect its flight dynamics. To maintain the desired trajectory, it is essential that the vehicle possess adequate control authority to account for these effects. As the HSV control surface is expected to have a strong influence on the dynamics of the vehicle, it is considered as a case study in this work. At each time step, the aerodynamic pressure loads and thermal loads cause the control surface to deform, leading to a change in the forces and moments acting on the vehicle and altering the trim state of the vehicle. As such, an iterative routine is employed whose objective is to quantify the control input necessary to account for control surface flexibility. The routine uses the control surface angle of attack, $\alpha$, as the control input and the lift produced by a rigid control surface, $L_{R}$, as the objective. The lift produced by a rigid control surface at particular flight conditions and a specific rigid angle of attack, $\alpha_{0}$, is first calculated. Aerothermoelastic simulation of the elastic control surface at these flight conditions is then carried out at $\alpha_{0}$. At pre-determined time intervals within the aerothermoelastic simulation, the analysis is paused and the deformed configuration at that time instant is stored. The deformed configuration is then used within the aerodynamic solver and the angle of attack is iterated on to minimize the residue, $\mathcal{R}$, given by

$$
\mathcal{R}=\left|L_{R}-L_{E}\right|
$$

where $L_{E}$ is the lift produced by the flexible (elastic) control surface. The residue is minimized by iterating on $\alpha$ using fmindbnd, an internal Matlab minimization routine. The angle of attack which minimizes the residue, $\alpha_{\min }$, is then stored at the current time instant and the simulation resumes at $\alpha_{0}$ to the next time instant. A flowchart of the iterative routine is given in Fig. 5. The introduction of such a routine provides insight as to the necessary robustness of the vehicle control effector under aerothermoelastic effects.

\section{Control Surface Model}

The hypersonic vehicle configuration considered in this study, as developed in a previous work, ${ }^{9}$ is shown in Fig. 6. A finite element model of a representative hypersonic vehicle elevator has been created for use in this study. The thickness from the top skin layer to the bottom skin layer is $4 \%$ chord length. ${ }^{70-72}$ The top and bottom skin layers are each equipped with two $3.8-\mathrm{mm}$ thick thermal protection system layers, and 


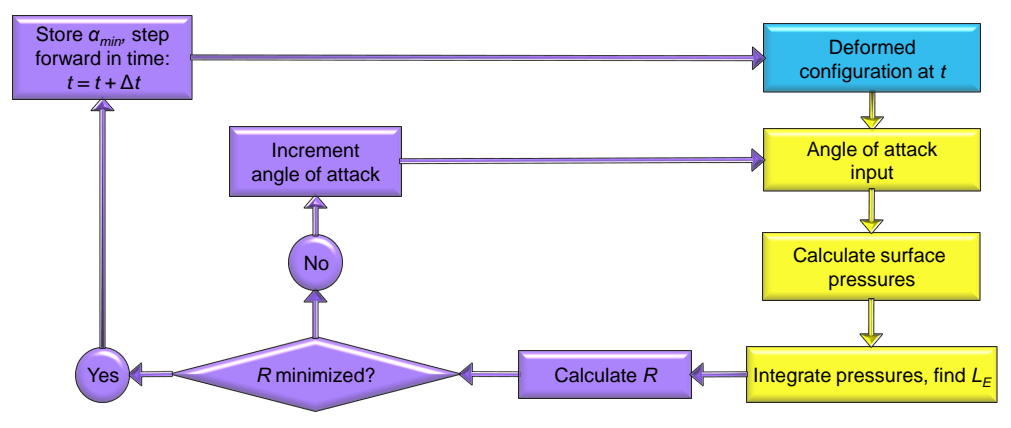

Figure 5. Flowchart illustrating the influence of aerothermoelastic effects on vehicle dynamics and control.

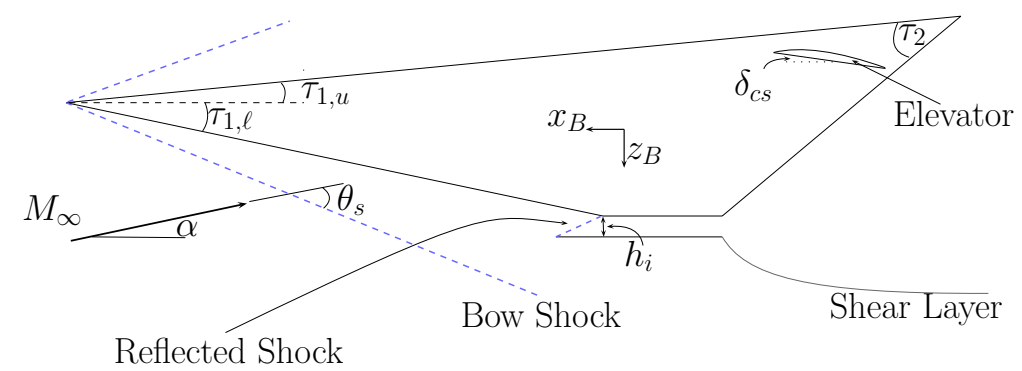

Figure 6. Overall HSV geometry illustrating position of control surface.

thus the thickness of the outer mold line is $4 \%$ chord length plus the 15.2 -mm of thermal protection system material. The chord length at the root is $5.2 \mathrm{~m}^{9}$ and the leading edge makes an angle of $34^{\circ}$ with the y axis while the trailing edge makes an angle of $18^{\circ}$ with the y axis. ${ }^{73}$ Planform and cross-sectional views of the airfoil are given in Fig. 7 and Fig. 8, respectively.

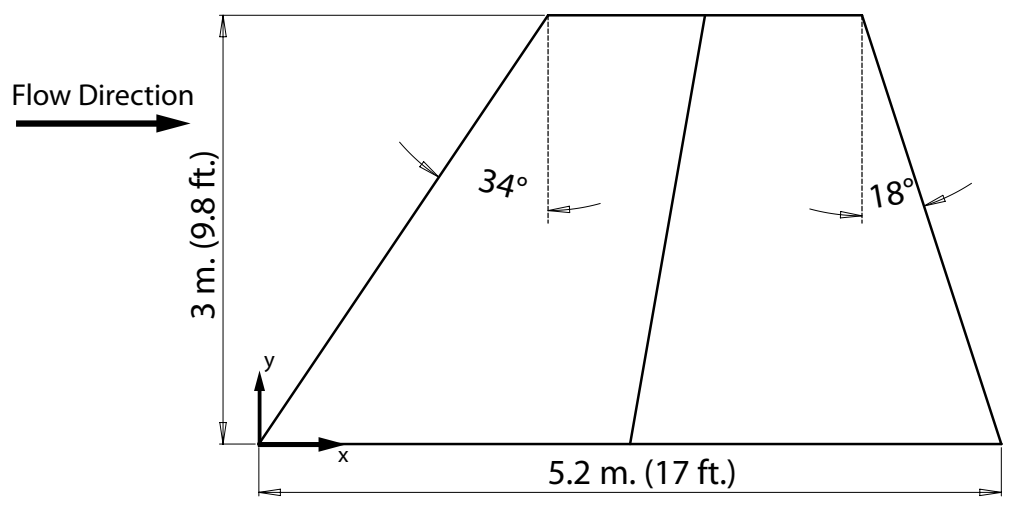

Figure 7. Planform geometry of control surface model.

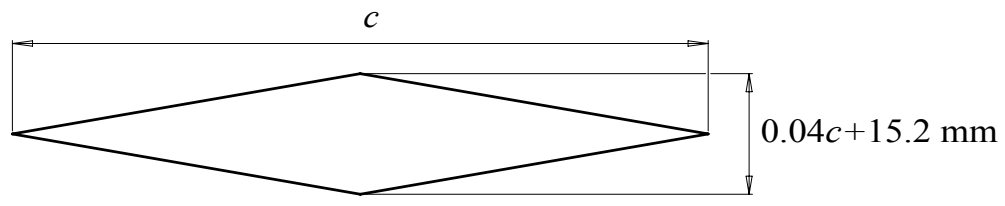

Figure 8. Cross-sectional geometry of control surface model.

A survey of the literature revealed a wide range of design strategies for mitigating the high temperatures experienced in hypersonic flight. ${ }^{74-81}$ This study considers a thermal protection system consisting of an outer heat shield and middle insulation layer on top of the structure as shown in Fig. 9. The material for 
the heat shield is chosen to be René 41 as it was found to be efficient in terms of mechanical properties at elevated temperatures. ${ }^{80}$ For the insulation layer, three different materials were considered in the preliminary materials evaluation: Internal Multiscreen Insulation (IMI) ${ }^{75}$ High Temperature Flexible Min-K, ${ }^{80}$ and Q-Fiber Felt. ${ }^{75}$ Of these, the Min-K insulation, which is a proprietary silica based material faced with Astroquartz cloth ${ }^{80}$ is selected due to its relatively low thermal diffusivity. For the structure, the Titaniumalloy TIMETAL 834 (formerly known as IMI 834) is chosen. The advantage of using this alloy is its relatively high maximum application temperature $\left(600^{\circ} \mathrm{C}\right)$ when compared with that of Ti-6242S $\left(520^{\circ} \mathrm{C}\right)$, Ti- 6242 $\left(450^{\circ} \mathrm{C}\right)$, Ti- $811\left(400^{\circ} \mathrm{C}\right)$, and Ti-6-4 $\left(300^{\circ} \mathrm{C}\right) .{ }^{82}$ The thermal and mechanical properties of the three materials employed in the model are given in Table 2, where "T-dep." indicates that the property is temperaturedependent. The emissivity of the heat shield is taken to be $0.85 .{ }^{73,83}$ The thermal strain is calculated based on the temperature change with respect to a reference stress-free temperature. The reference temperature used for calculation of thermal strain is taken to be $293 \mathrm{~K}$ for all materials.

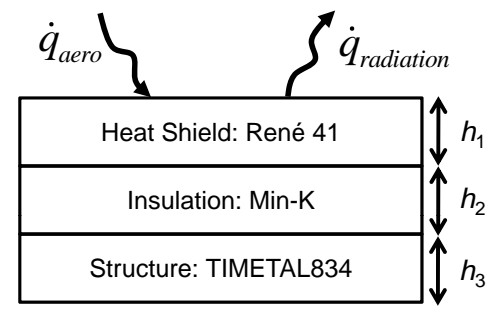

Figure 9. Schematic of material stacking scheme at outer mold line of structure.

Table 2. Structural and thermal material properties used in the study. ${ }^{73,80,84-86}$

\begin{tabular}{l||c|c|c|c|c|c|c|c}
\hline \hline & $\begin{array}{c}\rho \\
{\left[\mathrm{kg} / \mathrm{m}^{3}\right]}\end{array}$ & $\begin{array}{c}E \\
{[P a]}\end{array}$ & $\nu$ & $\begin{array}{c}\alpha_{T} \\
{[\mu \mathrm{m} / \mathrm{m} / \mathrm{K}]}\end{array}$ & $\begin{array}{c}k \\
{[W / \mathrm{m} / \mathrm{K}]}\end{array}$ & $\begin{array}{c}c_{p} \\
{[\mathrm{~J} / \mathrm{kg} / \mathrm{K}]}\end{array}$ & $\begin{array}{c}T_{\max } \\
K\end{array}$ & $\begin{array}{c}h \\
{[\mathrm{~mm}]}\end{array}$ \\
\hline René 41 & 8240 & T-dep. & 0.31 & T-dep. & 18 & 541 & 1500 & 3.8 \\
\hline Min-K & 256 & Neglect & Neglect & Neglect & 0.052 & 858 & 1250 & 3.8 \\
\hline TIMETAL 834 & 4550 & T-dep. & 0.31 & 11 & 7 & 525 & 873 & 6.35 \\
\hline \hline
\end{tabular}

The finite element model used for the thermal and structural modeling aspects of the study is shown in Fig. 10 with the top surface removed for visualization purposes. The model consists of the thermal protection layers system described above along with chordwise and spanwise stiffeners. The material used for the stiffeners is TIMETAL 834 and the thickness of all stiffeners is $25.4 \mathrm{~mm}$ (1 in). The model contains 2,812 thermal degrees of freedom and 8,074 structural degrees of freedom. The heat shield and insulation layer are each modeled using 6-node solid wedge elements while the top and bottom skins and stiffeners are modeled using 3-node, 2-dimensional triangular elements. Of the 6,886 elements in the model, 3,456 are solid elements and 3,430 are triangular elements. The control surface is taken to be all-moveable about a hinge line located at the mid-chord ${ }^{9}$ and will thus be connected to the vehicle main body through a torque tube. This attachment is modeled by constraining the region indicated by the gray circle in Fig. 10 in all degrees of freedom. In addition, the nodes at the root are constrained against translation in the y direction. Because the stiffness of the insulation layer is neglected, rigid (RBE2) elements are used between each skin node and the corresponding node at the outer surface of the insulation layer to prevent singularities in the solution.

The NASA Langley CFL3D code $^{87,88}$ is used in this study for full-order CFD solutions for the aerodynamic heating. The CFL3D code uses an implicit, finite-volume algorithm based on upwind-biased spatial differencing to solve the Reynolds-Averaged Navier-Stokes (RANS) equations. Multigrid and mesh-sequencing are available for convergence acceleration. The algorithm, which is based on a cell-centered scheme, uses upwind-differencing based on either flux-vector splitting or flux-difference splitting, and can sharply capture shock waves. The Menter $k-\omega \mathrm{SST}^{89}$ turbulence model is used in this study for closure of the RANS equations. Note that the applicability of this turbulence model in the hypersonic regime was verified recently in two separate studies. ${ }^{90,91}$ Also, note that CFL3D is an ideal gas code. Therefore, real gas effects are neglected in the present study. The heat flux in CFL3D is computed in the form of the Stanton number, as 


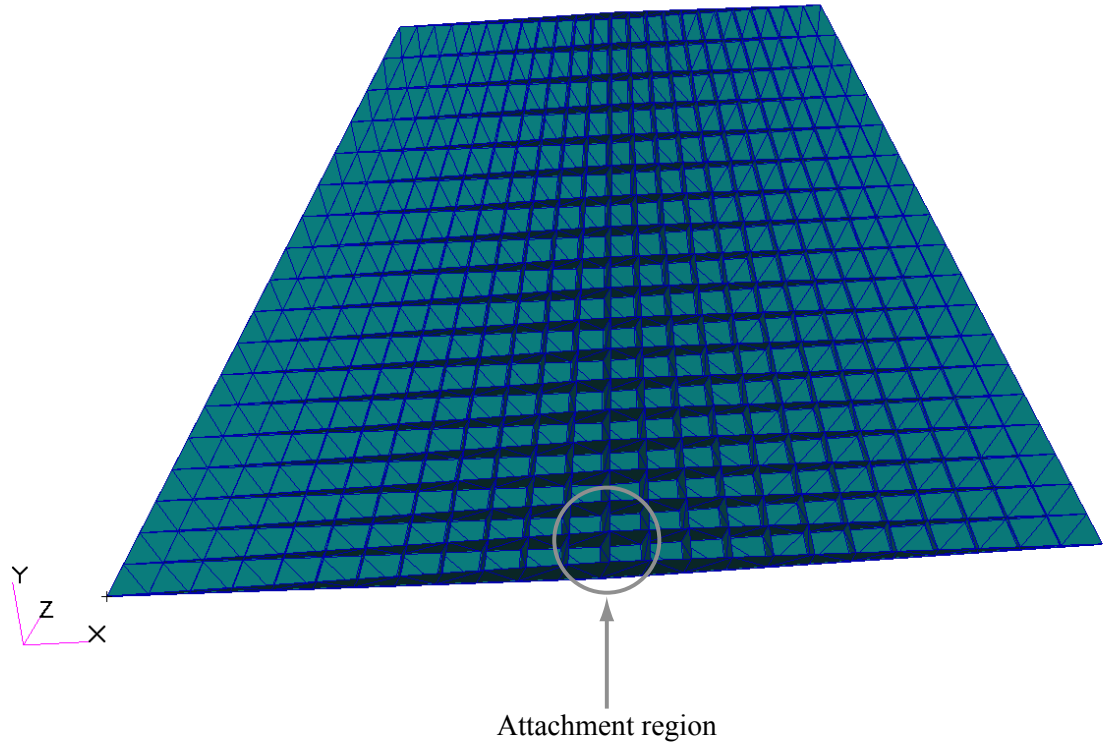

Figure 10. Finite element model of control surface used in study.

given by. ${ }^{87}$

$$
\dot{q}_{\text {aero }}=c_{p} V_{\infty} \rho_{\infty} S t\left(T_{w}-T_{t}\right),
$$

where $S t$ is the CFL3D Stanton number and $T_{t}$ is the total or stagnation temperature.

The fluid mesh for CFD computations, shown in Fig. 11, is an $\mathrm{H}-\mathrm{H}$ grid with 43 points spanwise, 109 points chordwise, and 49 points extending vertically from the surface $(435,000$ cells $)$. Note that cells are clustered near the surface, leading edge, and mid-chord since these locations correspond to maximum flow gradients. ${ }^{25}$ This grid does not include any flow sections upstream or downstream of the wing surface since the considered flow is hypersonic and disturbances cannot propagate upstream. Coefficients of lift and drag for this grid are within $0.05 \%$ and $0.25 \%$, respectively, of a similar 3.5 million cell grid.

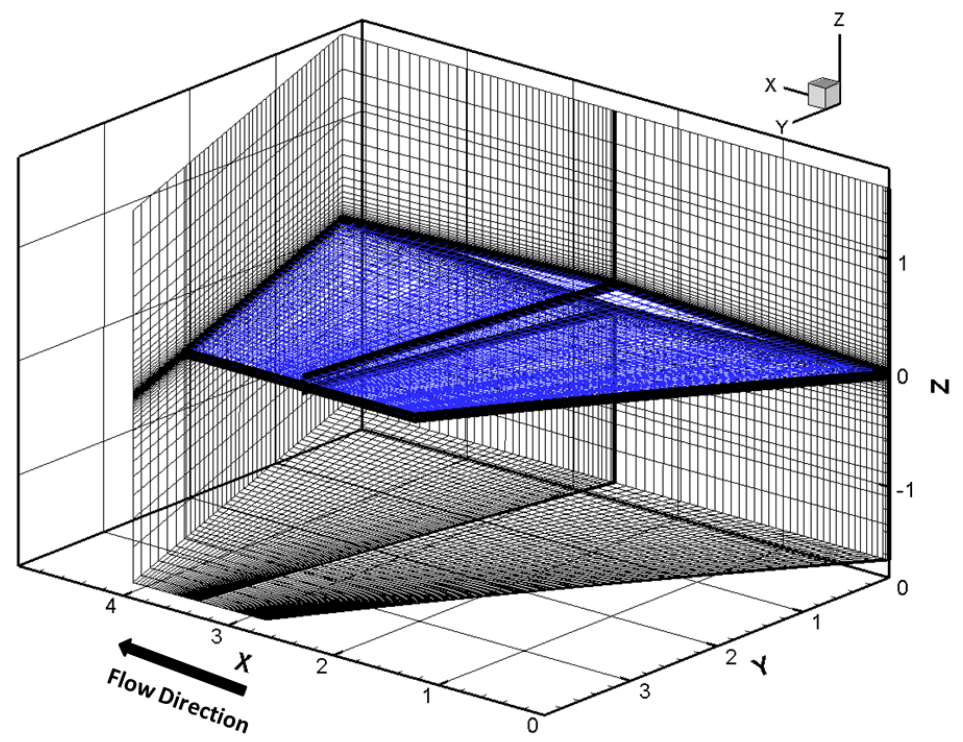

Figure 11. CFD model of control surface used in this study. 


\section{Results and Discussion}

\section{VIII.A. Selection of Thermal and Structural Modes}

In order to use the various ROMs in the aerothermoelastic solution, thermal and structural mode shapes must first be selected. The thermal POD modes are extracted from temperature snapshots which are representative of the expected dynamics in order for the basis to most closely span the space of the transient thermal solution throughout the simulation. The temperature snapshots are taken by sampling the transient temperature distribution every second from $0-5,000 \mathrm{~s}$ for the case of $\alpha=6^{\circ}$, Mach 8 , at an altitude of $26 \mathrm{~km}(85,000 \mathrm{ft})$. For this simulation, $\Delta t_{A E}$ and $\Delta t_{H T}$ are each $1 \mathrm{~s}$ and $\Delta t_{A T}$ is $4 \mathrm{~s}$. To calculate the aerodynamic heating in this simulation, the Eckert reference temperature method ${ }^{92,93}$ is used. The details of the implementation of the Eckert reference temperature method are given in a previous work. ${ }^{29}$ Using the 5,001 snapshots, the snapshot matrix is assembled, its SVD is taken, and the POD modes are extracted. The eigenvalues of the correlation matrix are found from the singular values of the snapshot matrix using Eq. (37). The first 24 eigenvalues of the correlation matrix are given in the semi-log plot shown in Fig. 12. Based on the magnitudes of the eigenvalues, the first 12 POD modes are used in the reduced-order thermal solution.

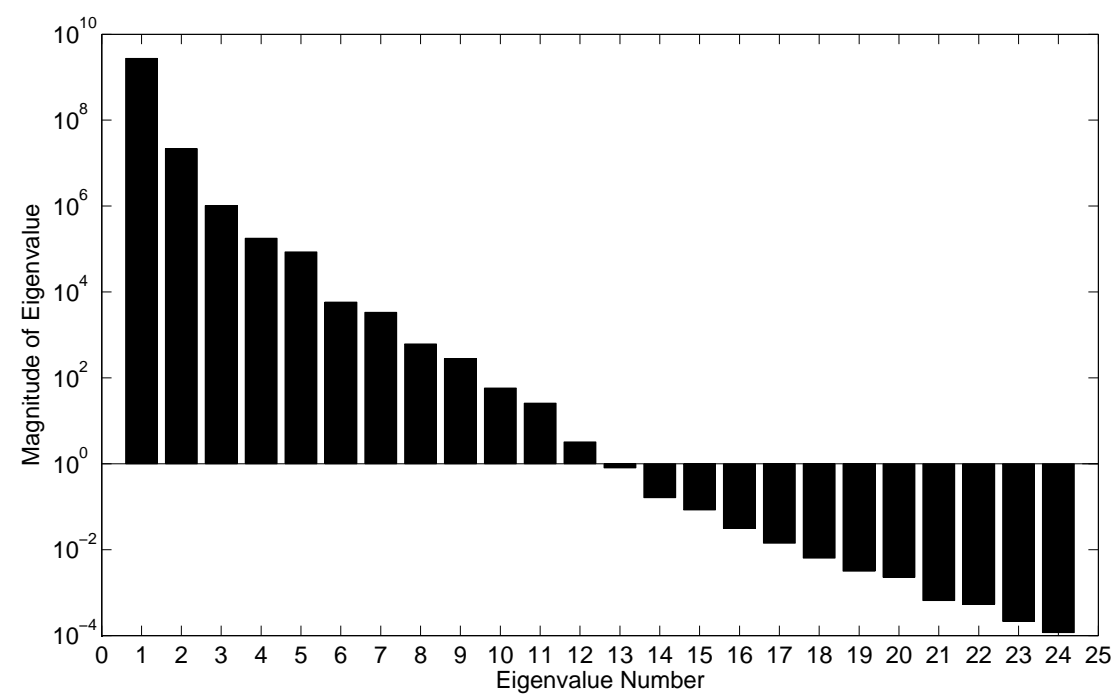

Figure 12. First 24 eigenvalues of correlation matrix.

The strategy for selecting the structural reference modes is to solve the free vibration problem at a reference thermal state that approximates the average temperature distribution (and thus, average stiffness distribution) over the length of a mission. These modes contain both the effect of material degradation with temperature as well as geometric stiffness effects due to thermal stresses. The thermal state at which to evaluate the structural reference modes is calculated by averaging the temperatures for each node of the model over the 5,001 snapshots used in generating the thermal modes. Contour plots showing the temperature distribution of the reference thermal state are given in Fig. 13. Although the maximum temperature on the bottom surface for the reference thermal state exceeds the maximum application temperature of the heat shield, temperature-dependent material property data has been extrapolated beyond this point and subsequent simulations will bound the flight parameters to ensure that the maximum temperature stays within feasible range.

The structural ROM utilizes the first six structural dynamic reference modes at the reference thermal state which are given in Fig. 14. The structural displacements are expected to be dominated by thermal loads early in the transient as the structure approaches thermal equilibrium and the bottom surfaces heats faster than the top surface for positive angles of attack. The thermal loads change on a slow time scale, and it is expected that the structural response to the thermal loads can be approximated in a quasi-static manner. Therefore, accuracy of the structural ROM is expected to increase by augmenting the original six-mode basis with a seventh mode that is computed based on the static deformed configuration due to the applied thermal loads in the reference thermal state. The inclusion of this mode is likely to capture the quasi-static response 


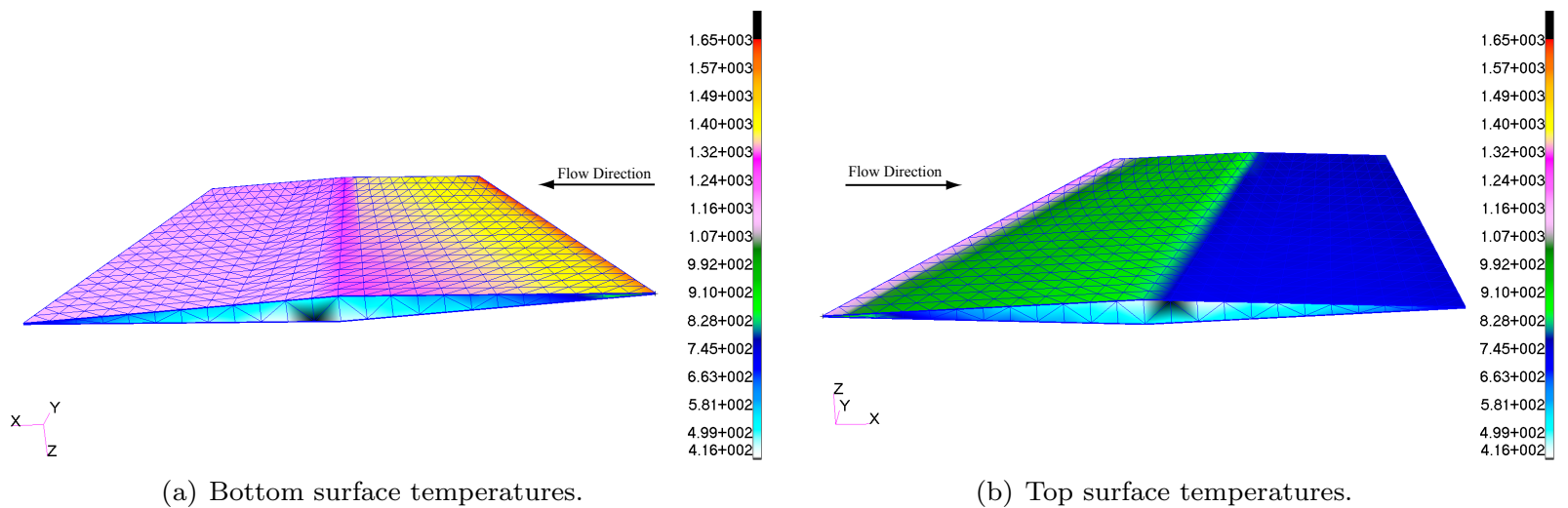

Figure 13. Temperature distribution $[\mathrm{K}]$ at reference thermal state over outer surfaces of structure.

due to slowly changing thermal loads which would otherwise not be captured with the original six structural dynamic eigenmodes. This additional seventh mode is shown in Fig. 14(g). To assess the effect of including this mode, structural ROM validation cases are run with both the six-mode and seven-mode bases.

\section{VIII.B. Summary of ROM Error Characterization}

To validate the various ROMs, sample aerothermoelastic cases are run to compare each ROM against the corresponding high-fidelity solution. The error incurred due to model reduction is quantified using two different error metrics. The first is the normalized root mean squared error (NRMSE). Expressed as a percentage, the NRMSE is given by

$$
\operatorname{NRMSE}[\%]=\frac{\sqrt{\frac{1}{s} \sum_{i=1}^{s}\left(\mathrm{ROM}_{i}-\mathrm{Full}_{i}\right)^{2}}}{\operatorname{Max}(\text { Full })-\operatorname{Min}(\text { Full })} \times 100,
$$

where $i$ is the $i$-th nodal value of interest, "ROM" represents a solution vector of the reduced-order model, "Full" represents a solution vector of the full-order model, and $s$ is the total number of data points in the solution vector. The second error metric to be used in validation of the reduced-order models is the $L_{\infty}$ error. Expressed as a percentage, the $L_{\infty}$ error is given by

$$
L_{\infty}[\%]=\frac{\operatorname{Max}(\mid \mathrm{ROM}-\text { Full } \mid)}{\operatorname{Max}(\text { Full })-\operatorname{Min}(\text { Full })} \times 100 .
$$

A summary of the average NRMSE and $L_{\infty}$ for each ROM is given in Table 3. Note that the average error is calculated differently for the thermal and structural ROMs than for the aerothermal ROM due to differences in the way the validation of each are carried out. For validation of the thermal and structural ROMs, aerothermoelastic simulations are run and the error of the ROM with respect to the corresponding full-order model is computed over the duration of the simulation as a function of time. Thus, the average errors given in Table 3 are calculated by averaging the corresponding error metric over the duration of the simulation. The case IDs over which the simulation errors are time-averaged are given in the table. The flight conditions corresponding to each case ID are detailed in the next section. Validation of the aerothermal ROM is carried out by generating 500 evaluation cases with the parameters chosen based on Latin Hypercube parameter space sampling. The average error of the aerothermal ROM is calculated by averaging each error metric over the 500 evaluation cases. The use of the 500 evaluation cases from Latin Hypercube Sampling is denoted in table as: "From LHS". As this is the first attempt to integrate the ROMs into a unified aerothermoelastic framework, specific areas for ROM improvement have been brought to light by this study. However, the primary focus of this paper is to implement the ROM framework and use it to assess the effect of aerothermoelasticity on HSV performance. Detailed analysis of the trade-off between number of aerothermoelastic states and ROM accuracy as well as development of techniques for improving the error of the ROMs is reserved for future work. 


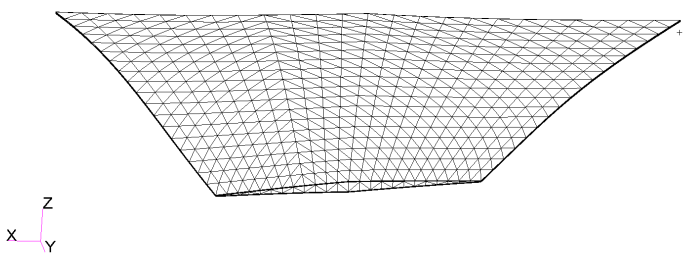

(a) Mode 1: $25 \mathrm{~Hz}$.

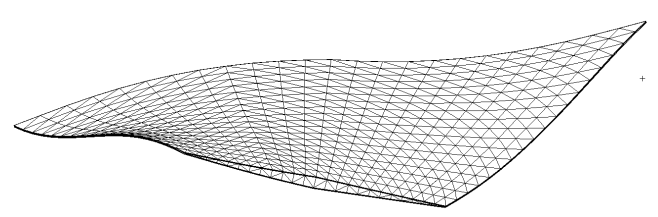

$\times{ }_{Y}^{Z}$

(c) Mode 3: $52 \mathrm{~Hz}$.

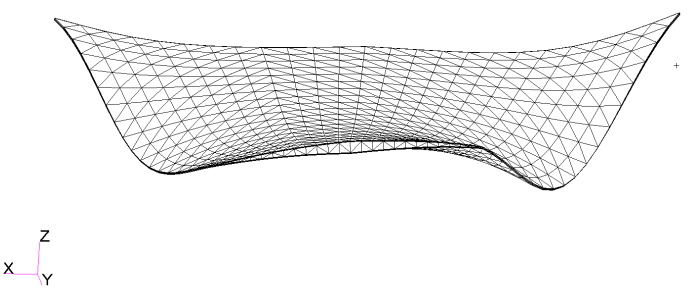

(e) Mode 5: $83 \mathrm{~Hz}$.

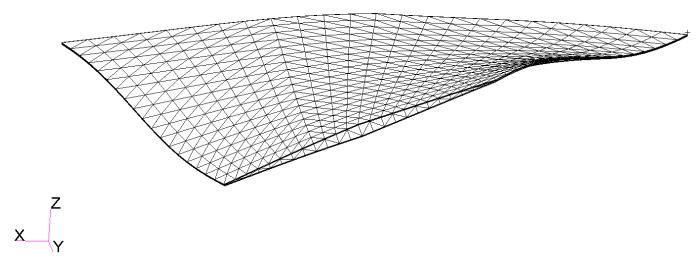

(b) Mode 2: $35 \mathrm{~Hz}$.

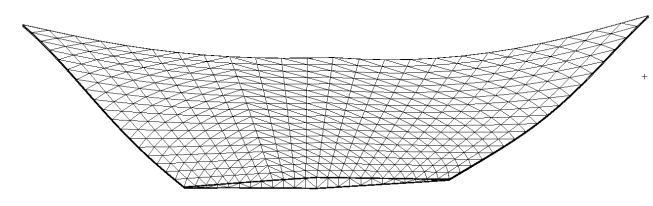

$z$

$x_{Y}$

(d) Mode 4: $54 \mathrm{~Hz}$.

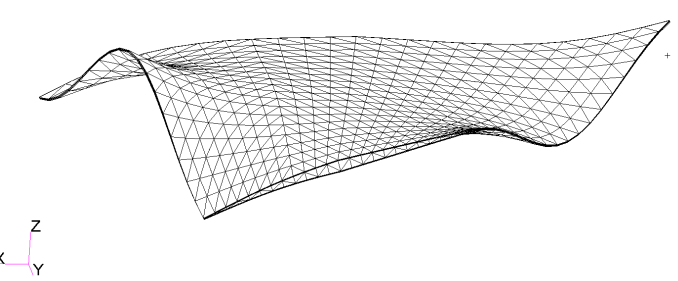

(f) Mode 6: $93 \mathrm{~Hz}$.

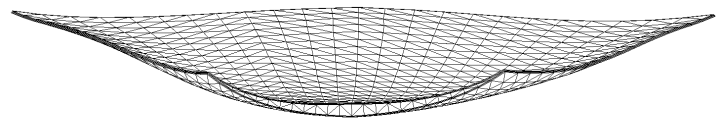

$$
z
$$

(g) Mode 7: Based on static deflection due to thermal loads in reference thermal state.

Figure 14. Structural mode shapes based on reference thermal state.

Table 3. Summary of average error of the various ROMs for selected cases.

\begin{tabular}{c|c|c|c}
\hline \hline ROM & Case & Average NRMSE [\%] & Average $L_{\infty}[\%]$ \\
\hline \multirow{2}{*}{ Thermal } & $1 \mathrm{RF}$ & 2.19 & 16.4 \\
& $2 \mathrm{RF}$ & 4.52 & 28.8 \\
\hline Structural & $4 \mathrm{FR}$ (7 Mode) & 8.52 & 28.7 \\
\hline Aerothermal & From LHS & 1.46 & 7.74 \\
\hline \hline
\end{tabular}




\section{VIII.B.1. Validation of Reduced-Order Thermal and Structural Models}

Two different flight conditions are used for validation of the thermal and structural ROMs. The altitude, Mach number, angle of attack, time step sizes, and initial temperatures for each case are summarized in Table 4. The first letter in the case ID specifies the thermal model used and the second letter specifies the structural model used where "R" denotes the ROM and "F" denotes the full-order model. Note that the full-order structural model is used in the thermal ROM validation cases (cases $1 \mathrm{RF}$ and $2 \mathrm{RF}$ ), and the full-order thermal model is used in the structural ROM validation cases (cases 1FR and 2FR). The Eckert reference temperature method is used to calculate aerodynamic heat flux for all thermal and structural ROM validation cases. The initial conditions for the thermal problem are a uniform temperature distribution of $293 \mathrm{~K}$ for all cases. Larger time steps are taken for the thermal ROM validation cases as the time scale of the heat transfer process is slower than that the of structural dynamics, and resolution of high-frequency structural dynamic oscillations is not of interest for thermal ROM validation.

Table 4. Parameters for aerothermoelastic simulations used to validate thermal and structural ROMs.

\begin{tabular}{l||c|c|c|c|c|c|c|c|c}
\hline \hline Case ID & Thermal Model & Structural Model & Alt. & $M_{\infty}$ & $\alpha$ & $\Delta t_{A E}$ & $\Delta t_{H T}$ & $\Delta t_{A T}$ & $T_{0}$ \\
\hline $1 \mathrm{RF}$ & ROM & Full-Order & $26 \mathrm{~km}$ & 8 & $3^{\circ}$ & $1 \mathrm{~s}$ & $1 \mathrm{~s}$ & $4 \mathrm{~s}$ & $293 \mathrm{~K}$ \\
\hline $2 \mathrm{RF}$ & ROM & Full-Order & $36 \mathrm{~km}$ & 6 & $1.5^{\circ}$ & $1 \mathrm{~s}$ & $1 \mathrm{~s}$ & $4 \mathrm{~s}$ & $293 \mathrm{~K}$ \\
\hline $1 \mathrm{FR}$ & Full-Order & ROM & $26 \mathrm{~km}$ & 8 & $3^{\circ}$ & $1 \mathrm{~ms}$ & $1 \mathrm{~ms}$ & $10 \mathrm{~ms}$ & $293 \mathrm{~K}$ \\
\hline $2 \mathrm{FR}$ & Full-Order & ROM & $36 \mathrm{~km}$ & 6 & $1.5^{\circ}$ & $1 \mathrm{~ms}$ & $1 \mathrm{~ms}$ & $10 \mathrm{~ms}$ & $293 \mathrm{~K}$ \\
\hline \hline
\end{tabular}

Validation of the thermal ROM is performed by running aerothermoelastic simulations on the control surface using the Eckert reference temperature formulation for the thermal boundary conditions with both the full-order and reduced-order thermal models. Plots of the time-history of the POD error for cases 1RF and 2RF are given in Figs. 15 and 16, respectively. The NRMSE percentage error shows good agreement with the full-order model in both cases. Based on the time-history of the $L_{\infty}$ error, it is evident that the thermal ROM has some difficulty capturing the steep temperature gradients within the initial portion of the transient. However, as the structure approaches thermal steady state and the temperature gradients and loads decrease, the accuracy of the thermal ROM improves.

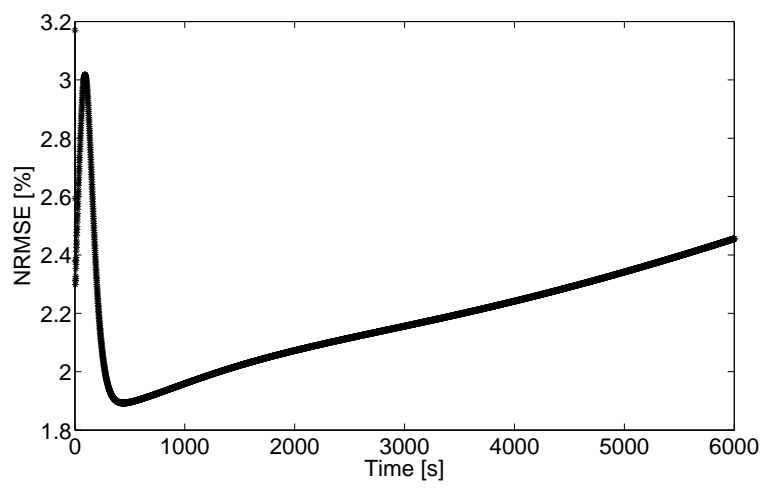

(a) NRMSE vs. time.

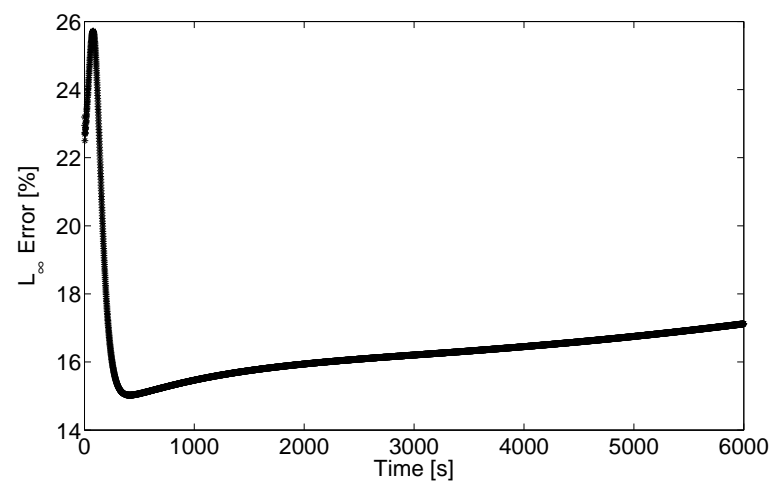

(b) $L_{\infty}$ error vs. time.

Figure 15. Error of POD thermal model for case 1RF based on nodal temperatures.

The structural ROM is compared against the high-fidelity structural model using the same two validation cases used for validation of the thermal ROM. The aeroelastic time step size, $\Delta t_{A E}$ is chosen based on the desire to resolve the structural reference modes with a minimum of 10 temporal sampling points for the highest frequency mode. The highest frequency of the chosen reference modes is $93 \mathrm{~Hz}$, however, recall that this mode was calculated at the reference thermal state with elevated temperatures. Thus, to account for the fact that the frequency of this mode may be slightly higher when the structure is at lower temperatures, $\Delta t_{A E}$ is chosen to be $1 \mathrm{~ms}$ based on a maximum frequency of $100 \mathrm{~Hz}$ with 10 temporal sampling points within one cycle. The heat transfer time step is chosen to be of the same size as the aeroelastic time step 


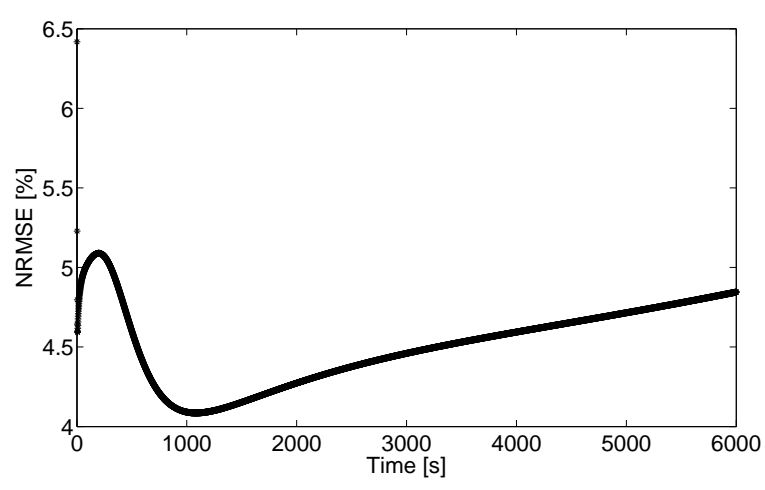

(a) NRMSE vs. time.

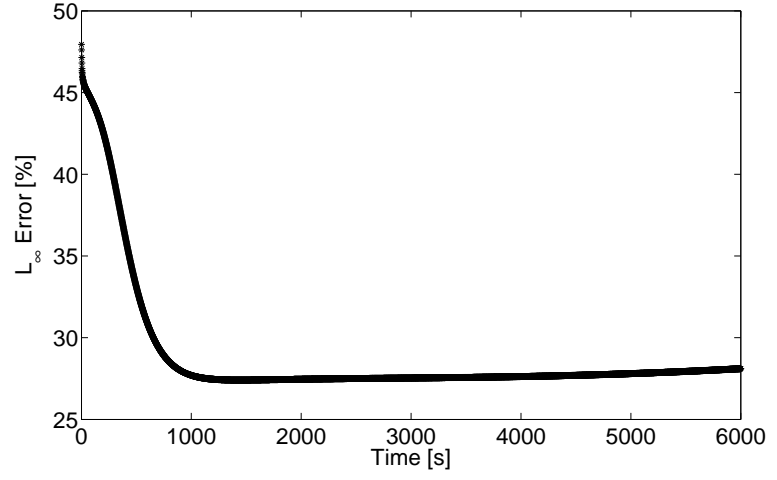

(b) $L_{\infty}$ error vs. time.

Figure 16. Error of POD thermal model for case 2RF based on nodal temperatures.

$\left(\Delta t_{H T}=\Delta t_{A E}\right)$ and the time between updates of the thermal boundary conditions, $\Delta t_{A T}$, is chosen to be $10 \mathrm{~ms}$. The solution vectors used to compute the difference between the high-fidelity and ROM solutions are vectors of the displacement in the $\mathrm{z}$ direction for the nodes at the outer surface of the finite element model at each time instant. Due to the small size of the time step and relatively long run times, the error is computed for the time range $0-6 \mathrm{~s}$. Plots of the time-history of the error for case 1FR using both the six-mode and seven-mode bases are given in Fig 17. The error for case 2FR is shown in Fig. 18. Both error metrics for both cases show an oscillating error in the initial part of the transient. This is due to initial high-frequency structural oscillations which are not captured by the structural ROM. As these oscillations are damped out, a decrease in the error of the structural ROM is observed. Note that these cases do not show a large difference in accuracy between the six-mode solution and seven-mode solution. This is likely due to the fact that the temperatures do not get high enough in the time range considered for the thermal loads to make a significant enough impact on the response. The structural dynamic response for longer time histories is studied next and will allow for further assessment of the difference between the six-mode and seven-mode structural ROMs at longer flight times.

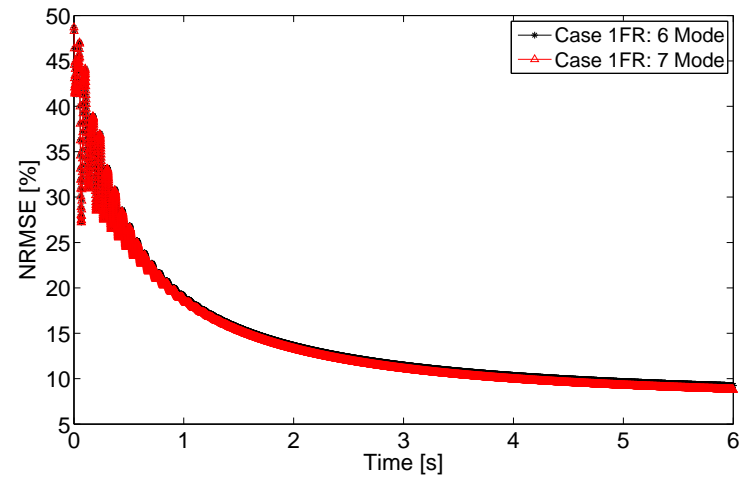

(a) NRMSE vs. time.

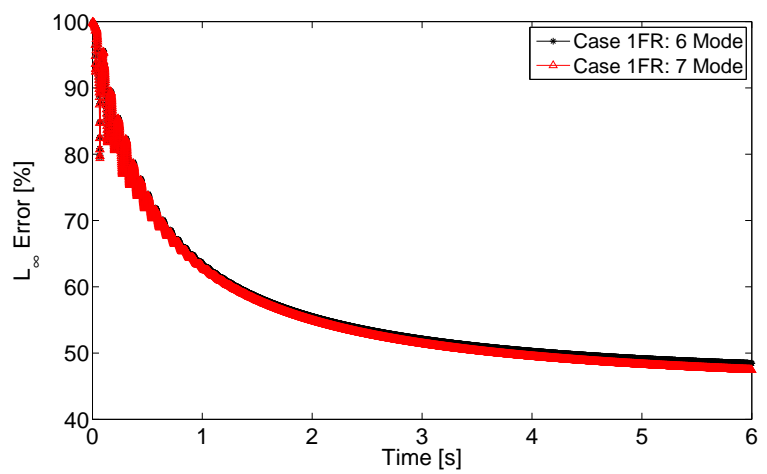

(b) $L_{\infty}$ error vs. time.

Figure 17. Error of structural ROM for case 1FR based on $\mathrm{z}$ displacements of surface nodes.

While a small aeroelastic time step size allows for the resolution of structural dynamic oscillations, in situations in which the thermal loads are dominant over the aerodynamic loads, it may be possible to capture the structural response with a larger aeroelastic time step because the thermal loads change on a much slower time scale than the aerodynamic loads. The largest heat flux in the above validation cases is most likely to occur in the initial transient of the simulation. This is due to the fact that the difference between the initial wall temperature of the structure and the initial recovery temperature is large, which leads to high aerodynamic heating. Furthermore, the difference between the initial wall temperature and the environment temperature is small which means that the heat flux radiated from the structure to the environment is small. Therefore, one might expect the highest thermal loads to occur in the initial part of the transient in 


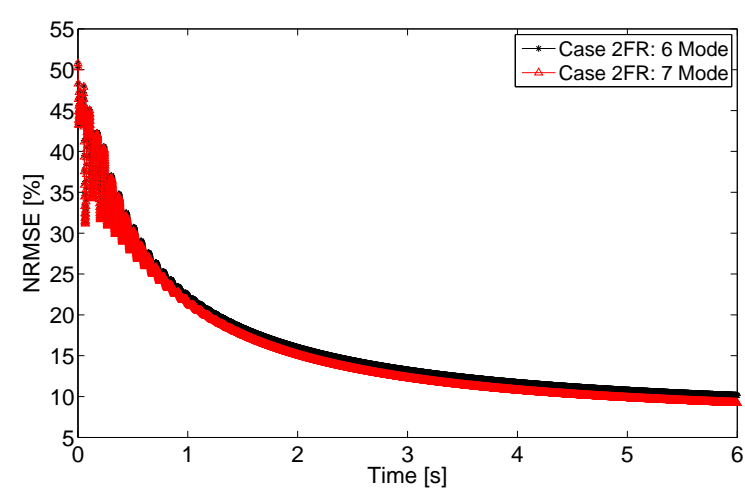

(a) NRMSE vs. time.

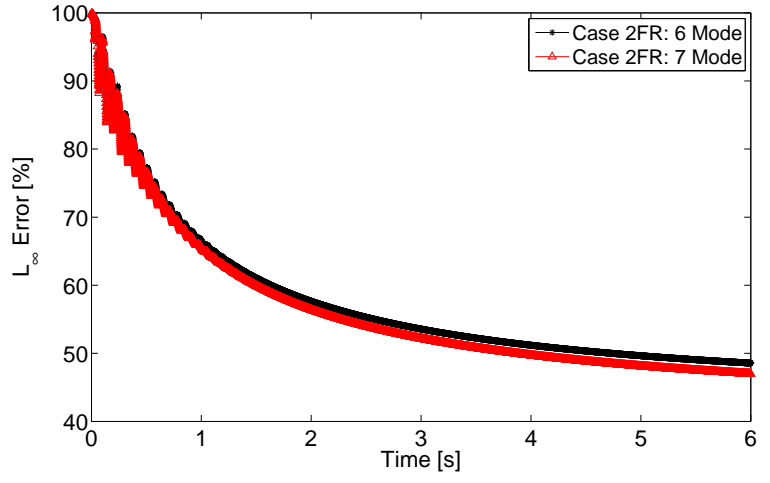

(b) $L_{\infty}$ error vs. time.

Figure 18. Error of structural ROM for case 2FR based on $\mathrm{z}$ displacements of surface nodes.

cases where the structure has a low initial temperature. To assess the effect larger time step sizes on the accuracy of the structural response, simulations are run with the full-order thermal and structural models using different time step sizes. These cases are denoted $3 \mathrm{FF}$ and $4 \mathrm{FF}$ and are summarized in Table 5 . Note that the time step sizes for case $4 \mathrm{FF}$ are each 100 times larger than those for case 3FF. Additionally, the difference in structural response between the full-order structural model and structural ROM for equal-sized larger size time steps is assessed using case $4 \mathrm{FR}$. This case is also summarized in Table 5.

Table 5. Parameters for aerothermoelastic simulations used to assess effect of time step size on structural response.

\begin{tabular}{l||c|c|c|c|c|c|c|c|c}
\hline \hline Case & Thermal Model & Structural Model & Alt. & $M_{\infty}$ & $\alpha$ & $\Delta t_{A E}$ & $\Delta t_{H T}$ & $\Delta t_{A T}$ & $T_{0}$ \\
\hline $3 \mathrm{FF}$ & Full-Order & Full-Order & $26 \mathrm{~km}$ & 8 & $3^{\circ}$ & $1 \mathrm{~ms}$ & $1 \mathrm{~ms}$ & $10 \mathrm{~ms}$ & $293 \mathrm{~K}$ \\
\hline $4 \mathrm{FF}$ & Full-Order & Full-Order & $26 \mathrm{~km}$ & 8 & $3^{\circ}$ & $0.1 \mathrm{~s}$ & $0.1 \mathrm{~s}$ & $1 \mathrm{~s}$ & $293 \mathrm{~K}$ \\
\hline $4 \mathrm{FR}$ & Full-Order & ROM & $26 \mathrm{~km}$ & 8 & $3^{\circ}$ & $0.1 \mathrm{~s}$ & $0.1 \mathrm{~s}$ & $1 \mathrm{~s}$ & $293 \mathrm{~K}$ \\
\hline \hline
\end{tabular}

A comparison of the $\mathrm{z}$ displacement of node 247 which is located at the midchord of the tip on the bottom surface is given for both cases in Fig. 19. Though case 4FF fails to capture the high-frequency oscillations due to the initial excitation because of its larger time steps, these oscillations are subsequently damped out and the nodal responses show good agreement following the initial oscillations. To assess the difference in $\mathrm{z}$ displacements between cases $3 \mathrm{FF}$ and $4 \mathrm{FF}$ for all nodes at the surface, the NRMSE and $L_{\infty}$ error are calculated for the time range $0.1-6 \mathrm{~s}$ in intervals of $0.1 \mathrm{~s}$. Case $3 \mathrm{FF}$ as treated as the reference and the errors are calculated based on the difference in the solution vectors from cases $3 \mathrm{FF}$ and $4 \mathrm{FF}$ at each time instant. The plots of the error given in Fig. 20 show good agreement between the two solutions beyond approximately $1 \mathrm{~s}$. The error of the structural ROM for larger time steps is assessed by computing the NRMSE and $L_{\infty}$ error between cases $4 \mathrm{FF}$ and $4 \mathrm{FR}$. The use of larger time steps allows for error quantification over a longer time record for the same amount of computational time. Plots of the error as a function of time are given in Fig. 21. Improvement of the structural ROM by addition of the seventh mode is observed in Fig. 21 after approximately $50 \mathrm{~s}$. This is likely due to the fact that by this time the temperatures have increased to the point where the thermal loads have a significant impact on the structural response. Though the initial NRMSE of case $4 \mathrm{FR}$ is approximately $39 \%$, it improves to below $10 \%$ within $5 \mathrm{~s}$. The displacement histories of node 247 for cases $4 \mathrm{FF}$ and $4 \mathrm{FR}$ with both the six-mode and seven-mode models are given in Fig. 22 to illustrate the level of structural displacements for these flight conditions.

\section{VIII.B.2. Validation of Reduced-Order Aerothermal Model}

Since this work represents an initial step in modeling the coupled aerodynamic heating/heat transfer and aerodynamic heating/structural displacement response of a hypersonic control surface, the number of modes required from the heat transfer and structural reduced-order models in order to accurately model the aerodynamic heating is uncertain. Thus, as a first step in accessing the aerothermoelastic response of the control surface, the first five thermal POD modes and the first five free vibration structural modes are included in 


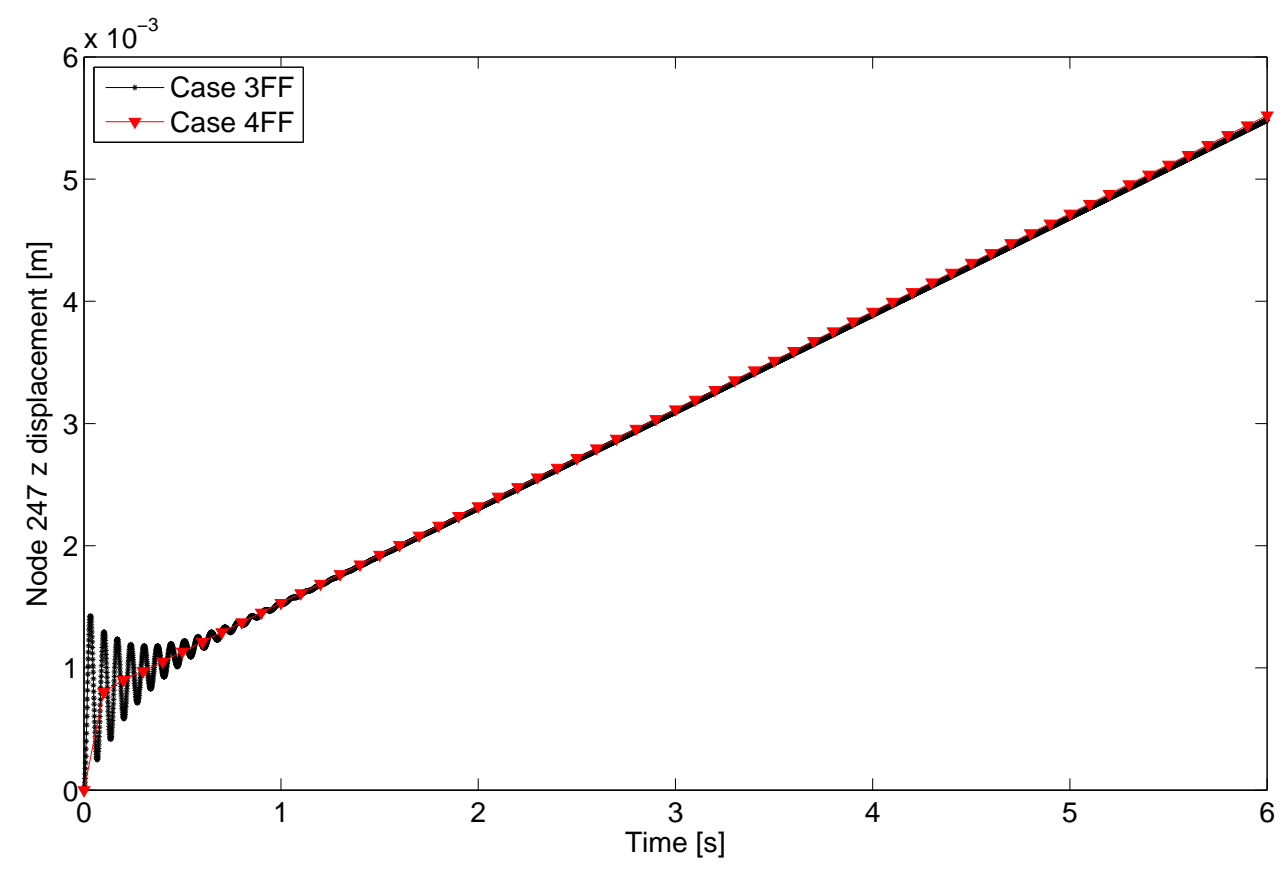

Figure 19. Node $247 \mathrm{z}$ displacements: case $3 F F$ vs. case $4 F F$

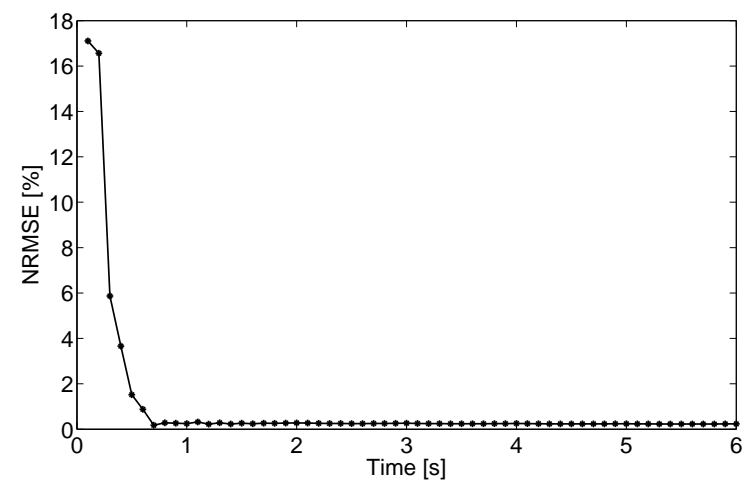

(a) NRMSE vs. time.

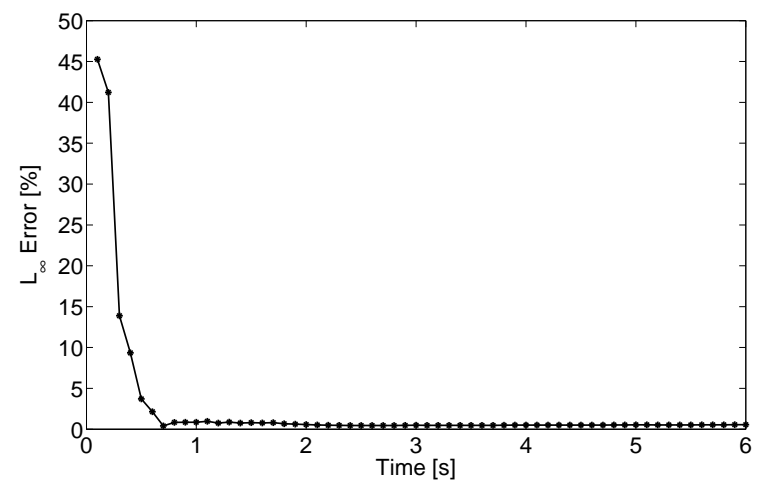

(b) $L_{\infty}$ error vs. time.

Figure 20. Error of case 3FF with respect to case $4 \mathrm{FF}$ based on $\mathrm{z}$ displacements of surface nodes.

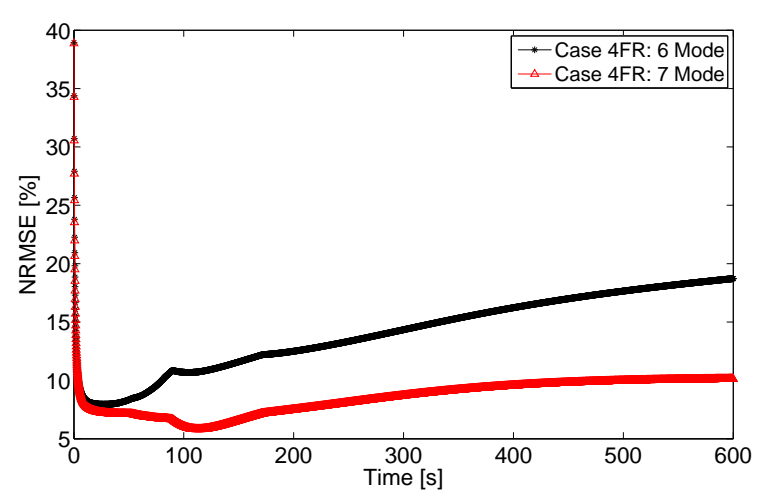

(a) NRMSE vs. time.

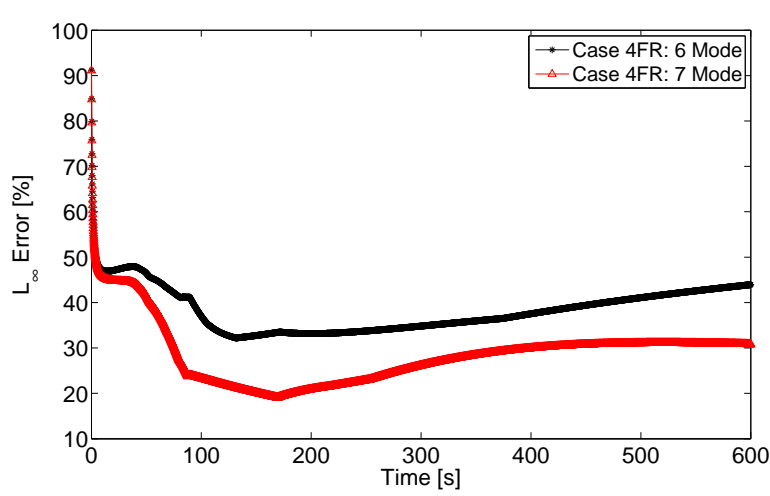

(b) $L_{\infty}$ error vs. time.

Figure 21. Error of case $4 \mathrm{FR}$ with respect to case $4 \mathrm{FF}$ based on $\mathrm{z}$ displacements of surface nodes. 


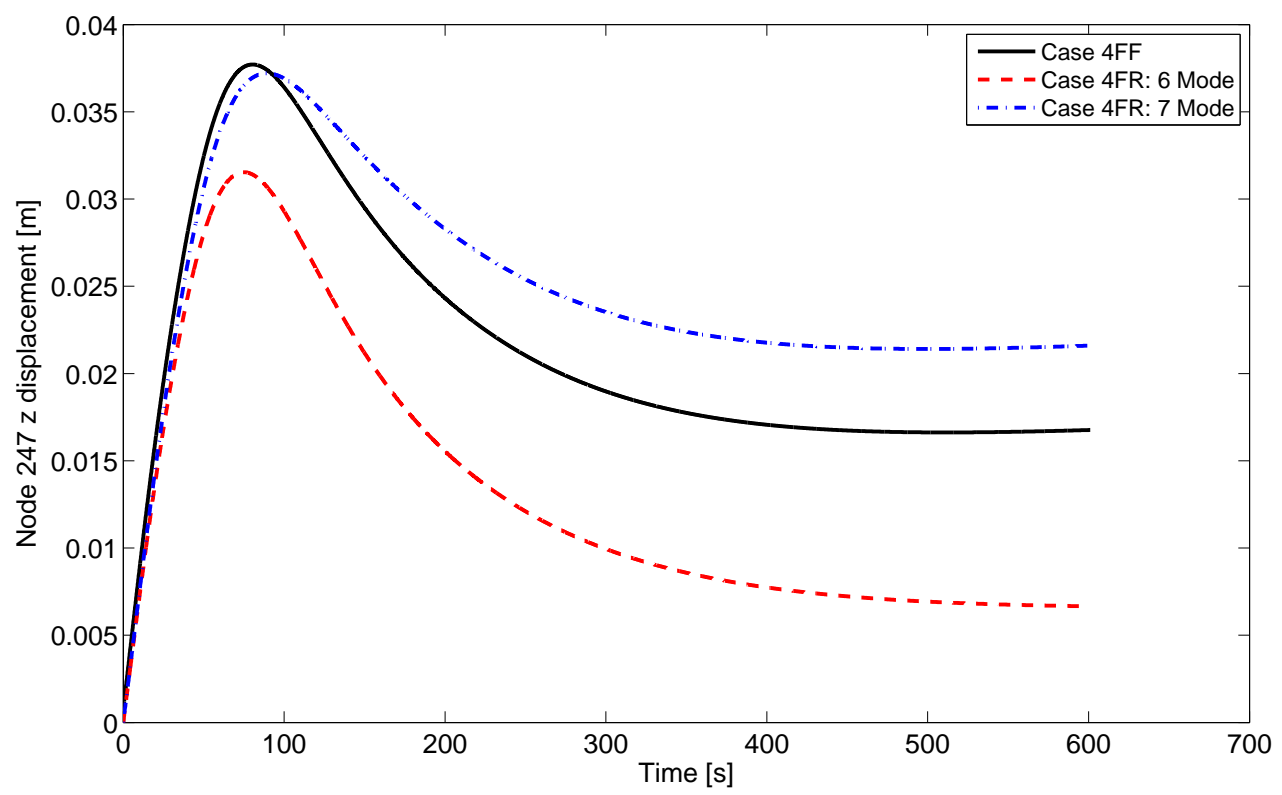

Figure 22. Node $247 \mathrm{z}$ displacements: case $4 \mathrm{FF}$ vs. case $4 \mathrm{FR}$.

\section{the ROAM.}

Previous work ${ }^{34}$ has demonstrated the need for a large number of snapshots to accurately capture the aerodynamic heat flux on a hypersonic control surface. Thus, 2500 snapshots are selected through LHS for the parameter space defined in Table 1. 2000 snapshots are used to construct the kriging ROAM, and 500 are used to evaluate the ROAM accuracy. The NRMSE and $L_{\infty}$ error metrics are used to illustrate the accuracy of the ROAM. These error metrics are computed for each evaluation case, resulting in a vector of 500 NRMSE and $L_{\infty}$ values for the ROAM. In order to provide a scalar quantification of the model quality, the average NRMSE and average $L_{\infty}$ over the 500 evaluation cases is computed, and the overall max $L_{\infty}$ of the 500 is identified. The average NRMSE is $1.46 \%$, the average $L_{\infty}$ is $7.74 \%$, and the overall maximum $L_{\infty}$ at any point on the control surface for all 500 evaluations is $27.54 \%$.

In order to gain insight into the sources of error for the present problem, two additional error metrics are computed as a function of surface location. As before, both average and maximum error metrics are considered. In Eq. (83), the absolute value of the percent error at each surface location is averaged over the total number of evaluation cases, $\bar{E}$ :

$$
\bar{E}[\%]=\left(\frac{1}{500} \sum_{j=1}^{500} \frac{[\mid \text { ROAM }- \text { Full } \mid]_{j}}{[\operatorname{Max}(\text { Full })-\operatorname{Min}(\text { Full })]_{j}}\right) \times 100
$$

where $j$ refers to the $j$-th evaluation case. In order to identify the locations where maximum errors most often occur, the surface location of the $L_{\infty}$ error for each evaluation case is recorded. The total number of times an $L_{\infty}$ error occurs at a given location is then tabulated.

These error metrics as a function of surface location are shown in Figs. 23(a-d) for the ROAM. Several general observations can be made from these results. First, it is clear that the average error as a function of surface location, shown in Figs. 23(a,c), is generally small. The average error over the entire control surface is generally less than $2 \%$. Furthermore, the average error is generally highest near the leading edge of the control surface. Note that these surface regions are also most likely to correspond with the maximum errors over the control surface, as shown in Figs. 23(b,d).

\section{VIII.C. Influence of Aerothermoelastic Effects on Aerodynamic Forces}

With the error of the reduced-order models characterized, these models are now used within the aerothermoelastic framework to assess the effect of aerothermoelasticity on the total lift and drag forces acting on the control surface. All simulations from this point forward use the corresponding ROM for the aeroheating, 


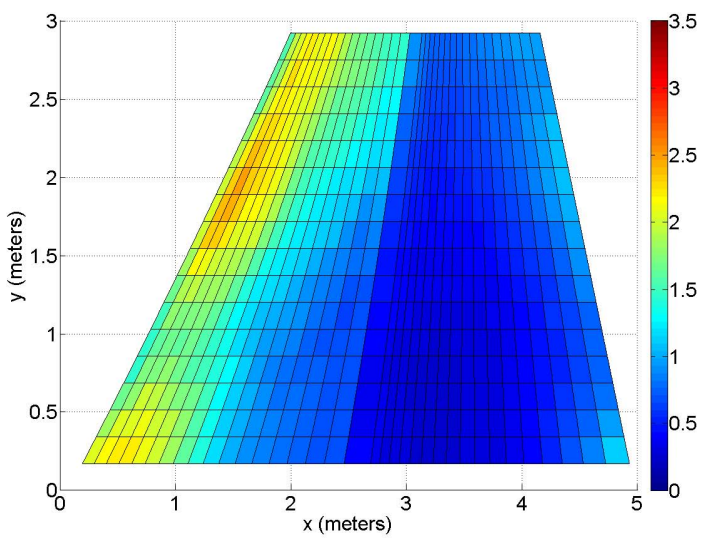

(a) ROAM average error (\%), top surface.

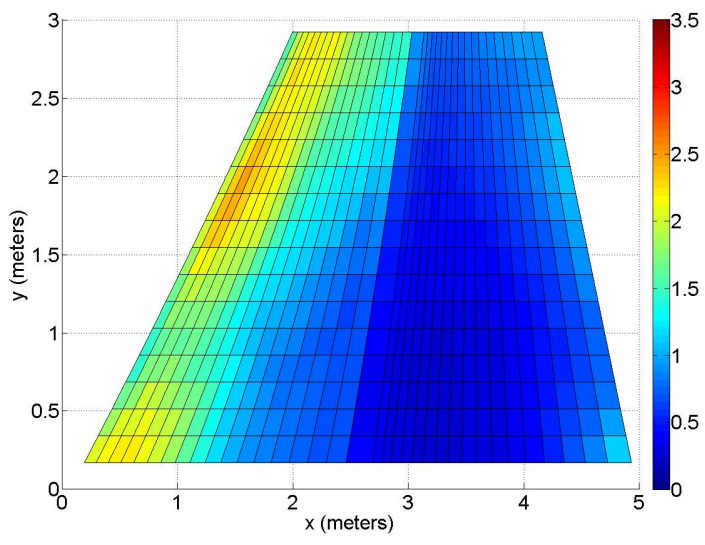

(c) ROAM average error (\%), bottom surface.

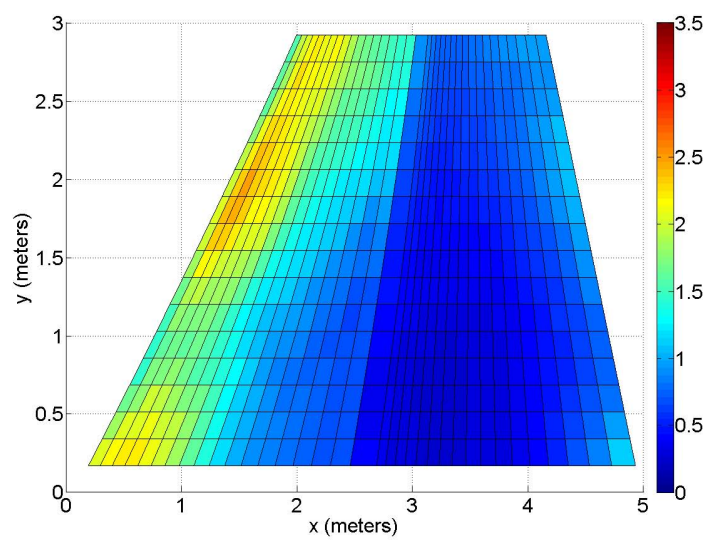

(b) Locations of maximum ROAM $L_{\infty}$, top surface.

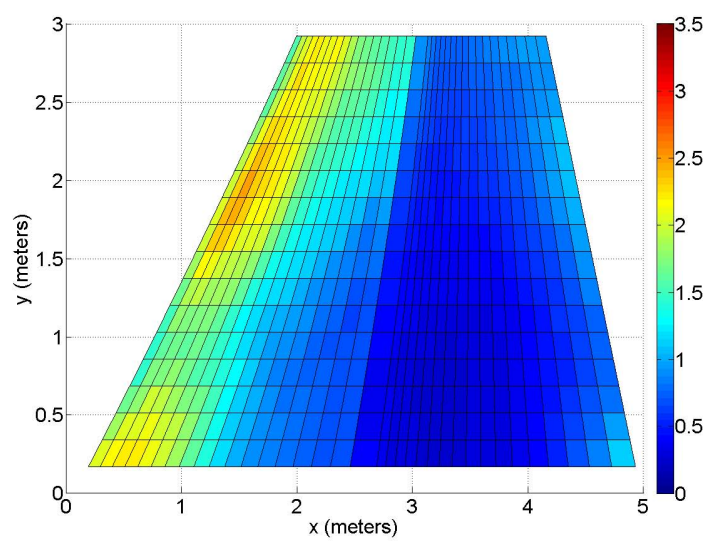

(d) Locations of maximum ROAM $L_{\infty}$, bottom surface.

Figure 23. Spatial distribution of ROAM errors for the 500 test cases. 
heat transfer, and structural dynamic response components. For these simulations, aeroelastic iterations are carried out to bring the structure to aeroelastic equilibrium prior to beginning the aerothermoelastic simulation. To find the total aerodynamic forces, the pressures are integrated over the outer surfaces of the structure. The pressures consist of a component in the direction of the local normal to the deformed configuration due to aerodynamic pressures and a component in the direction of the local tangent to the deformed configuration due to viscous stress. The viscous stress is computed using local skin friction coefficients for each finite element using an Eckert reference temperature formulation. ${ }^{29}$ The element-uniform pressure is found by averaging the nodal pressures for each finite element and multiplying by each element area for both the normal pressures and shear stress. Vectors normal and tangent to each element are calculated in the deformed configuation and each element pressure is resolved into components in the lift and drag directions. Total lift and drag are found by summing the contributions from each element over the outer surfaces of the model.

The time-marching aerothermoelastic cases used for this part of the study are summarized in Table 6 , where "LD" denotes that these cases are used for lift and drag assessment. Cases 1LD and 2LD represent constant-Mach, constant- $\alpha$ cruise at an initial uniform stress-free temperature of $293 \mathrm{~K}$. These cases are run for longer time-histories $(0-1,200 \mathrm{~s})$ to allow the temperature distribution to more fully evolve and to assess the response over a wide range of thermal conditions. Cases 3LD and $4 \mathrm{LD}$ are based on the ascent phase of a proposed trajectory for a single-stage-to-orbit mission of a blended wing body configuration typical of a transatmospheric hypersonic vehicle. ${ }^{79}$ Curve fits to the data points given in Ref. 79 are used to obtain the time-dependence of the altitude and Mach number and two different angles of attack are selected. As the ROMs in this work are valid for a specific range of flight parameters, only the Mach 5 - Mach 10 portion of the ascent trajectory is considered. Difficulty arises in determining the initial temperatures for the Mach 5 - Mach 10 ascent because the ROMs do not permit simulation from take-off to Mach 5. Thus to obtain the initial temperatures, a cruise condition at the initial altitude $(25 \mathrm{~km})$ and initial Mach number (Mach 5) is simulated for $163 \mathrm{~s}$ which is the amount of time the vehicle takes to reach Mach 5 for the given trajectory. This initial temperature distribution is denoted as " 163 s cruise" in the table. After the initial cruise segment, the temperature distribution is stored for use as the initial temperature distribution for the ascent segment, time is reset to zero, and the ascent trajectory is commenced. As the simulation time for these cases is shorter $(0-172 \mathrm{~s})$, the corresponding time step sizes for these cases are chosen to be smaller.

Table 6. Cases for assessment of transient lift and drag.

\begin{tabular}{c||c|c|c|c|c|c|c}
\hline \hline Case & Alt. $[\mathrm{km}]$ & $M_{\infty}$ & $\alpha$ & $\Delta t_{A E}[\mathrm{~s}]$ & $\Delta t_{H T}[\mathrm{~s}]$ & $\Delta t_{A T}[\mathrm{~s}]$ & $T_{0}$ \\
\hline 1LD & 26 & 8 & $3^{\circ}$ & 0.1 & 0.1 & 1 & $293 \mathrm{~K}$ unif. \\
\hline 2LD & 36 & 6 & $1.5^{\circ}$ & 0.1 & 0.1 & 1 & $293 \mathrm{~K}$ unif. \\
\hline 3LD & $-7 \times 10^{-5} t^{2}+0.06 t+24.97$ & $0.03 t+5$ & $3^{\circ}$ & 0.01 & 0.01 & 0.1 & 163 s cruise \\
\hline 4LD & $-7 \times 10^{-5} t^{2}+0.06 t+24.97$ & $0.03 t+5$ & $1.5^{\circ}$ & 0.01 & 0.01 & 0.1 & 163 s cruise \\
\hline \hline
\end{tabular}

First, the total lift force, $L$, and drag force, $D$, are calculated over the undeformed (rigid) control surface at initial flight conditions for each case. Aerothermoelastic simulation for the elastic control surface is then carried out for each case and total lift and drag are calculated at each aeroelastic time step. Plots of the transient lift and drag force are given in Figs. 24 and 25, respectively, for both the rigid and elastic control surfaces. Note that the rigid case contains no aerothermoelastic effects and the skin friction is computed at initial flight conditions at a uniform temperature of $293 \mathrm{~K}$. To assess the relative impact of flexibility on the total forces, the percentage differences between the lift and drag of the elastic structure are calculated with respect to the lift and drag produced by the rigid structure. Results for the relative change lift and drag are given in Figs. 26 and 27, respectively. For cases 1LD and 2LD, there is an initial abrubt change in the lift and drag forces due to the high temperature gradients as the structure begins to heat from room temperature and approach equilibrium temperature. At the end of the time-history shown for these cases, the total lift and drag begin to level off as the temperature gradients in the structure decrease. For ascent trajectory represented by cases $3 \mathrm{LD}$ and $4 \mathrm{LD}$, both the lift and drag are monotonically decreasing for the complete duration of the simulation. The initial abrubt change in the aerodynamic forces is not present in these cases because the initial temperature distribution is much higher than that used for cases 1LD and 2LD. Though the trend of the elastic and rigid control surfaces is similar for both lift and drag in cases 3LD and $4 \mathrm{LD}$, the percentage difference between rigid and elastic drag is more pronounced than that for the lift. 
Note that the initial percentage change in lift and drag for cases 3LD and $4 \mathrm{LD}$ is nonzero due to the fact that the aerothermoelastic solution is converged at cruise prior to beginning the simulation such that the structure is in a thermoelastically deformed state at initial time (see Fig. 25(c)-(d)).

To assess the level of heating for each of the cases, the maximum nodal temperature is plotted as a function of time for each of the cases. Results are given in Fig. 28. For cases 1LD and 2LD, there is a steep increase in the maximum temperature in the initial portion of the transient as the structures heats up from room temperature. As the structural approaches equilibrium temperature, the gradient in the maximum temperature begins to decrease. For the ascent trajectory of cases $3 \mathrm{LD}$ and $4 \mathrm{LD}$, we see a monotonic increase in maximum temperature for the time range considered. For all cases, the maximum temperature remains below $1,500 \mathrm{~K}$.

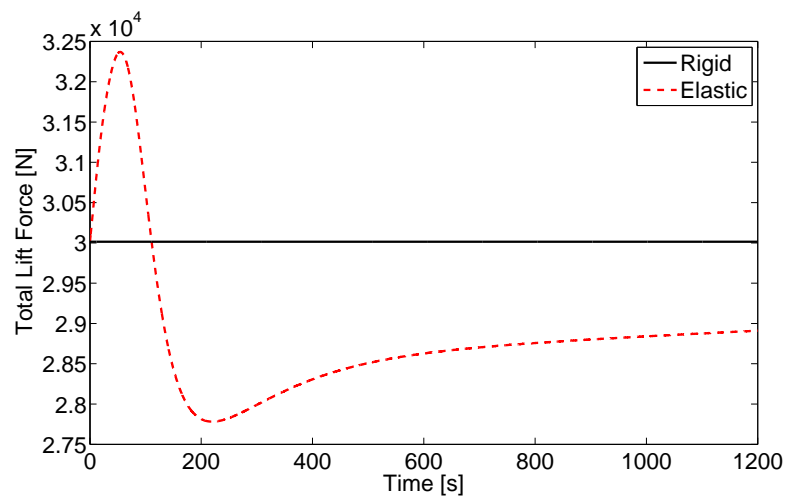

(a) Case 1LD.

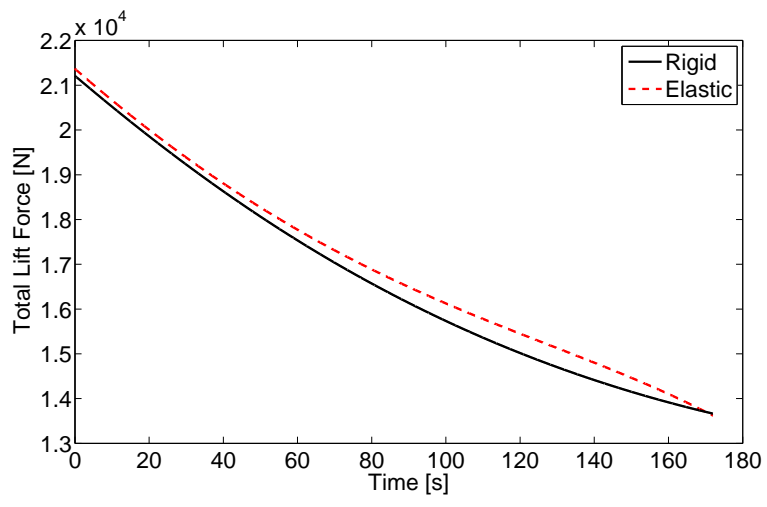

(c) Case 3LD.

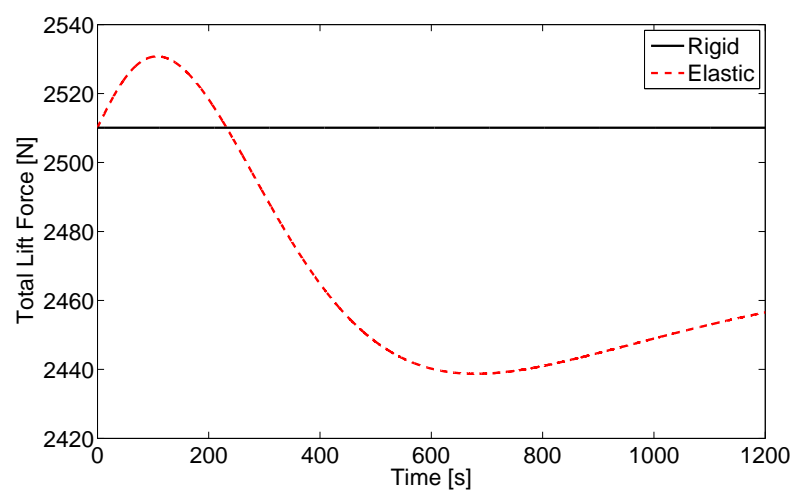

(b) Case 2LD.

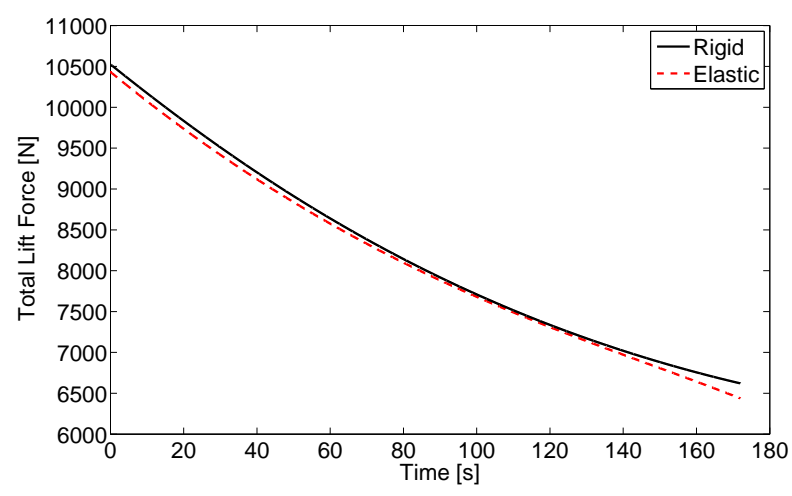

(d) Case $4 \mathrm{LD}$.

Figure 24. Time-history of total lift force for various flight conditions.

To determine the effect of aerothermoelasticity on aerodynamic performance and necessary control input, the iterative routine described in Section VI is incorporated into the aerothermoelastic framework. Cases $1 \mathrm{LD}$ and $2 \mathrm{LD}$ are used as example cases and $\alpha_{\min }$ is updated and stored every time the thermal boundary conditions are updated. Thus, $\alpha_{\min }$ is updated every 10 aeroelastic iterations (once per second) based on the relative time step sizes used for these cases. Recall that the initial angle of attack is $3^{\circ}$ for case $1 \mathrm{LD}$ and $1.5^{\circ}$ for case $2 \mathrm{LD}$. Plots of the time-history of $\alpha_{\min }$ for the two cases are given in Fig. 29. Note that the maximum residue, $\mathcal{R}$, is $0.3 \mathrm{~N}$ for case $1 \mathrm{LD}$ and $0.04 \mathrm{~N}$ for case $2 \mathrm{LD}$. As seen in the figures, the maximum absolute departure of $\alpha_{\min }$ from the initial angle of attack is $7.4 \%$ for case 1LD and $2.8 \%$ for case 2LD. However, note that the largest departures from initial angle of attack occur early in the transient which is likely an effect of the low initial temperature and resultant large temperature gradients. For cases in which the initial temperature of the structure is higher, the deformation due to thermal loads will be lower and the impact on overall lift and drag will be less. 


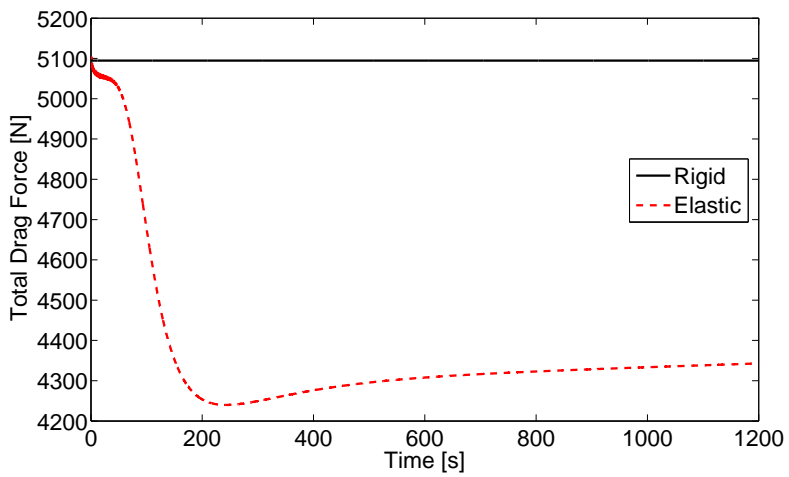

(a) Case 1LD.

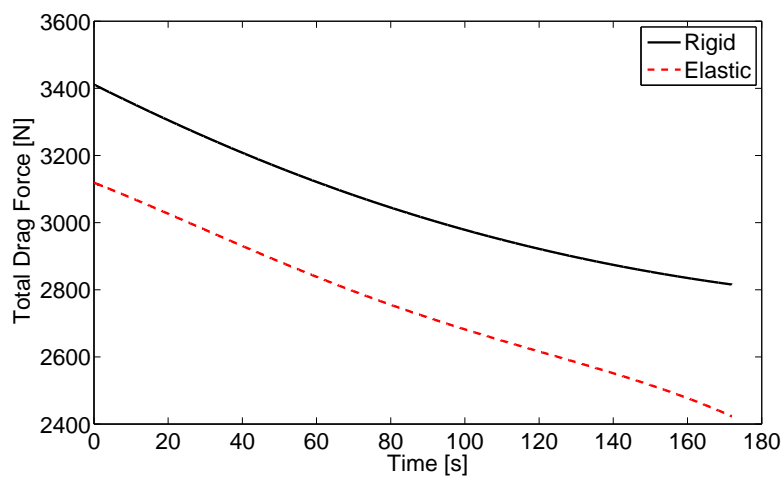

(c) Case 3LD.

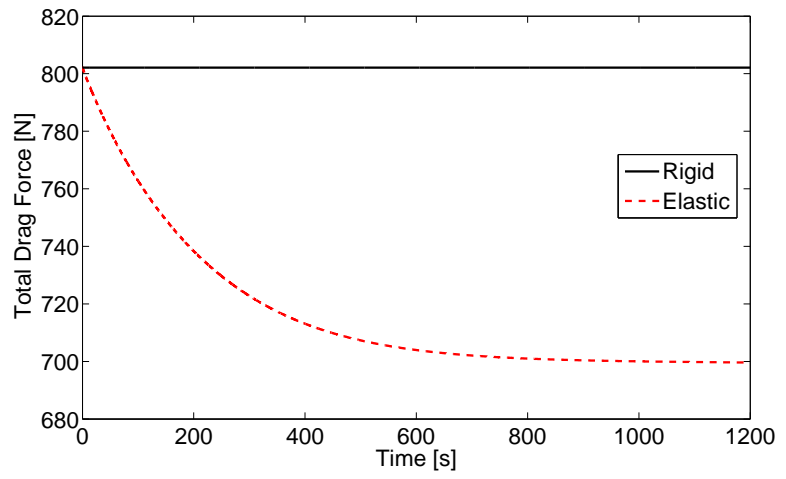

(b) Case 2LD.

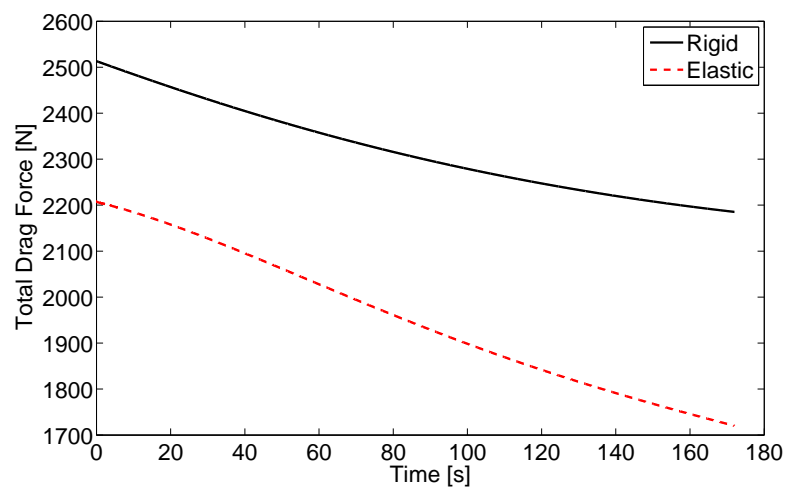

(d) Case 4LD.

Figure 25. Time-history of total drag force for various flight conditions. 


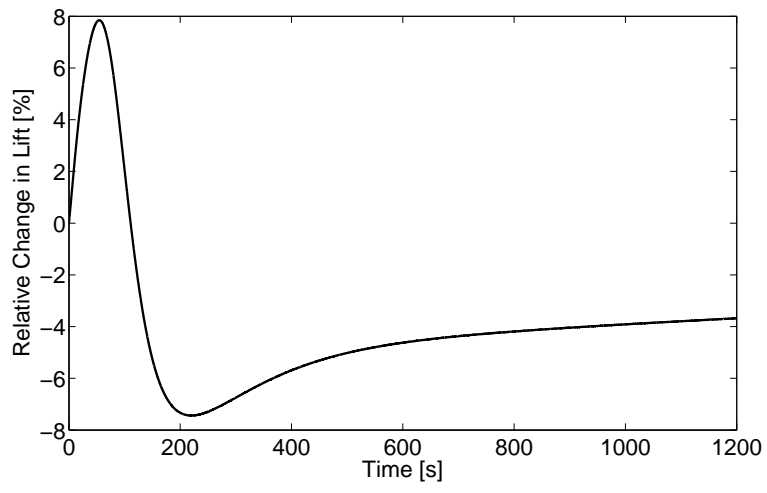

(a) Case 1LD.

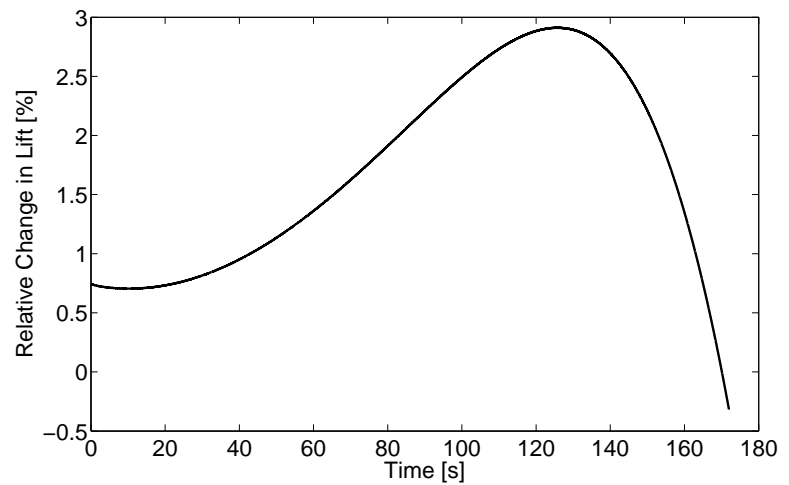

(c) Case 3LD.

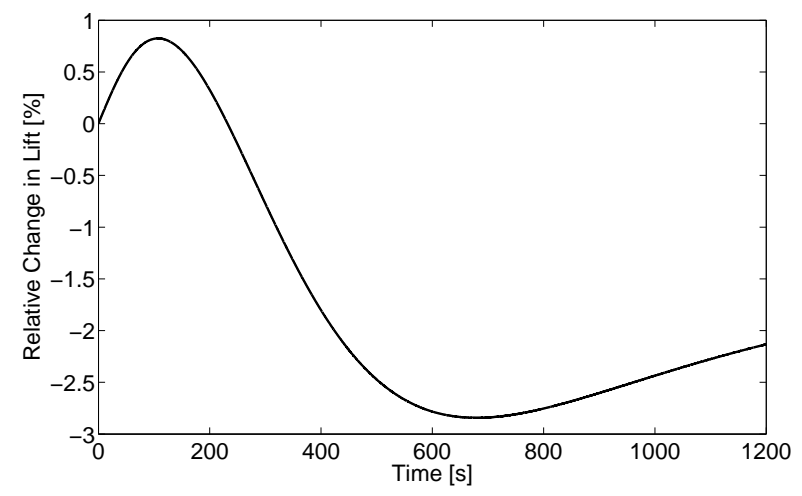

(b) Case 2LD.

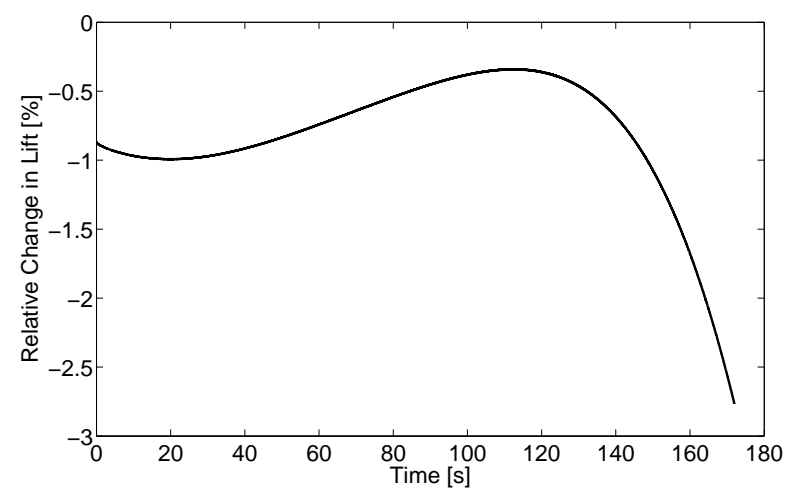

(d) Case 4LD.

Figure 26. Time-history of percentage change in lift force for various flight conditions. 


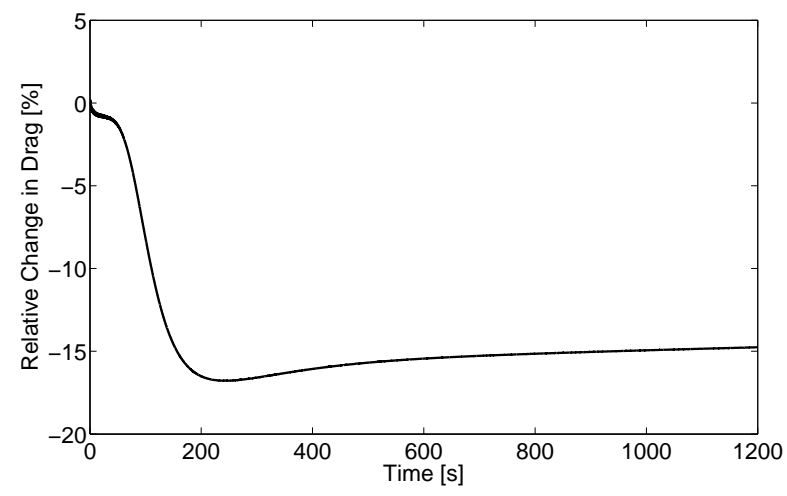

(a) Case 1LD.

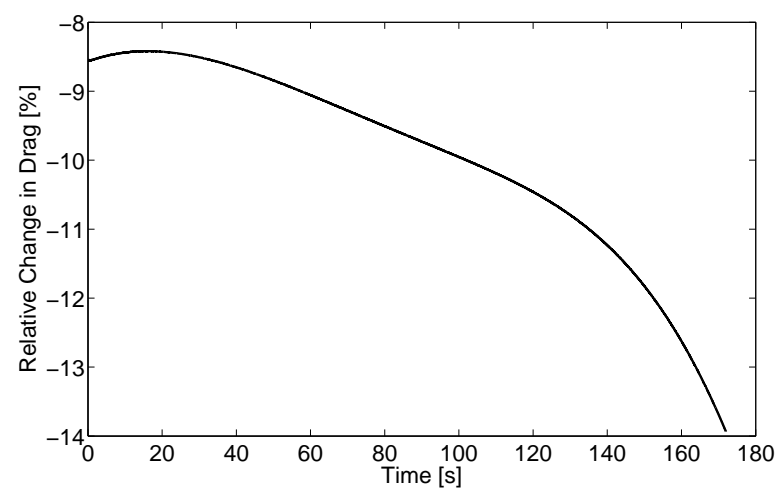

(c) Case 3LD.

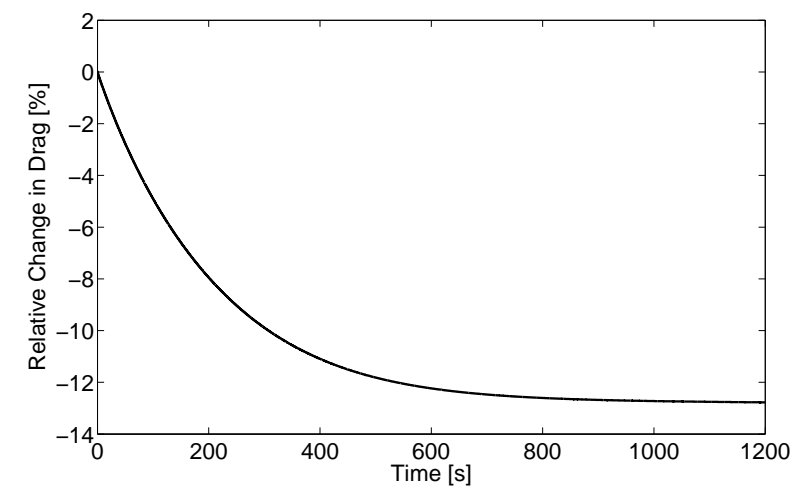

(b) Case 2LD.

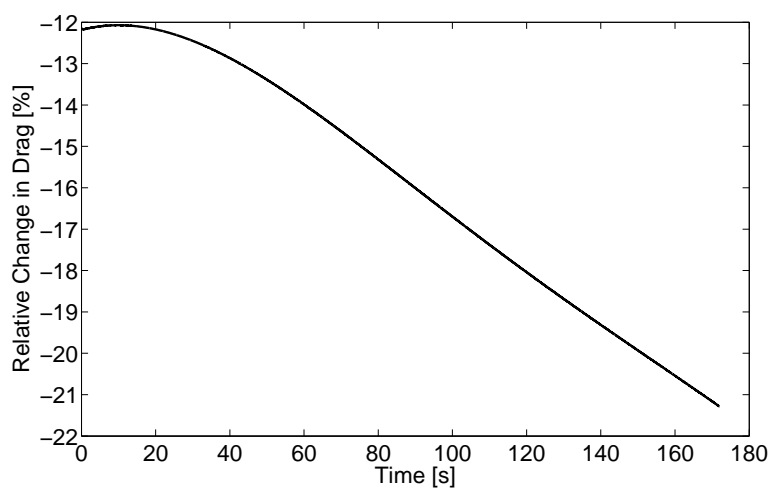

(d) Case 4LD.

Figure 27. Time-history of percentage change in drag force for various flight conditions. 


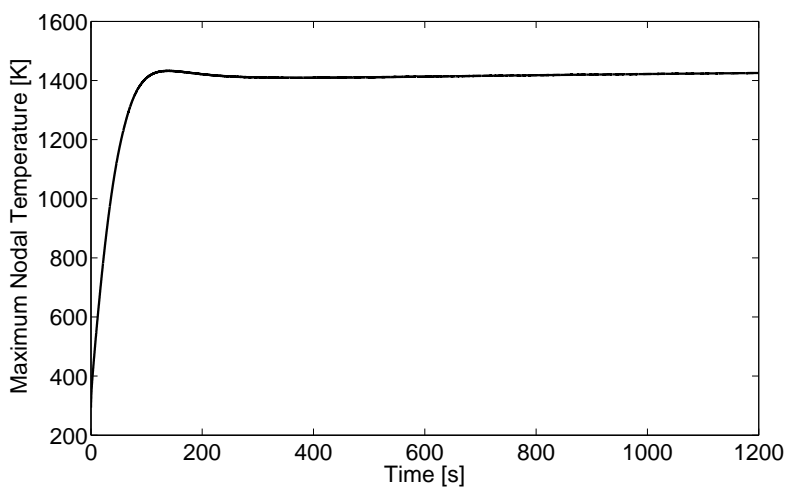

(a) Case 1LD.

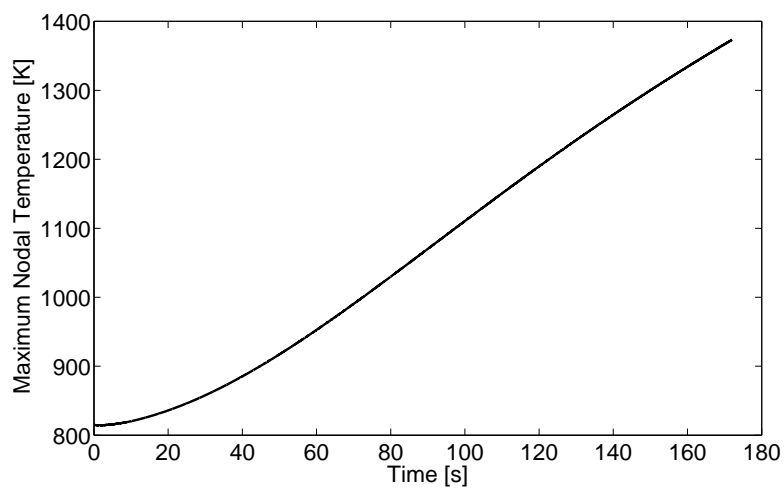

(c) Case 3LD.

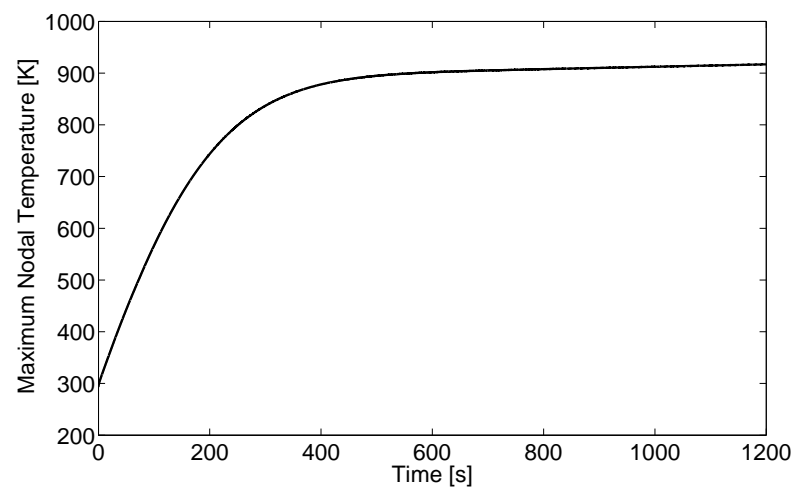

(b) Case 2LD.

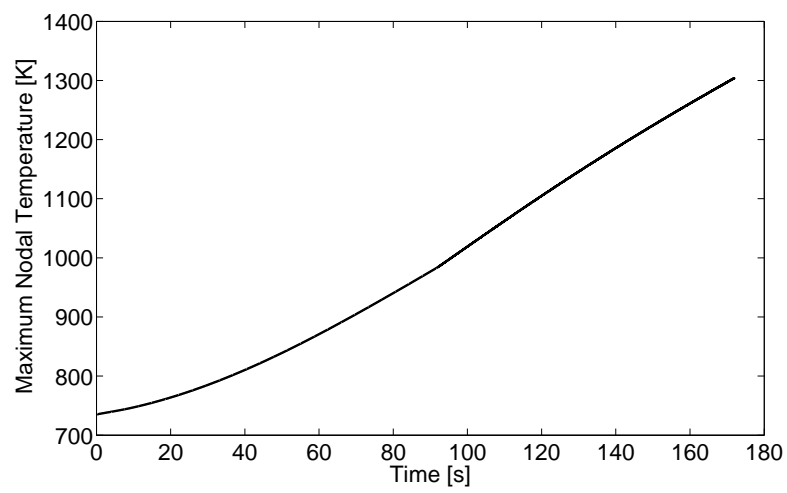

(d) Case 4LD.

Figure 28. Time-history of maximum nodal temperature for various flight conditions.

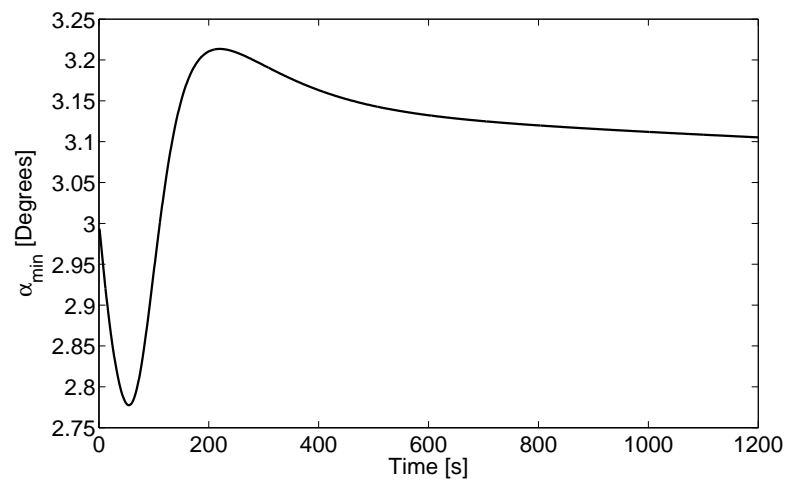

(a) Case 1LD.

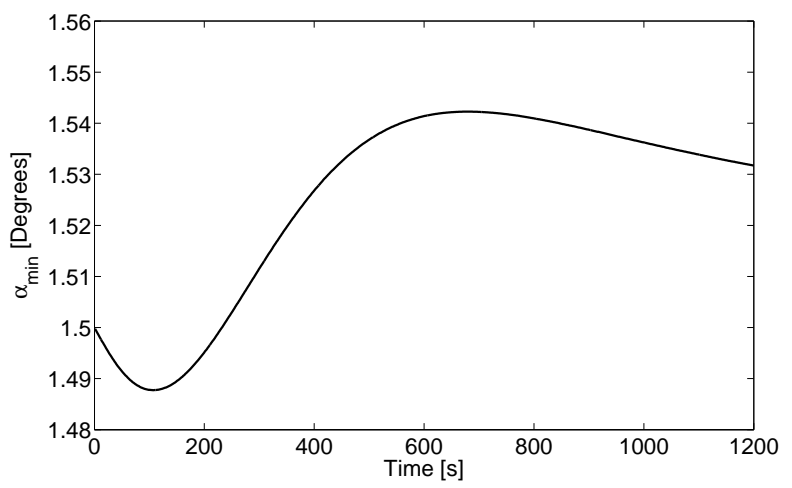

(b) Case 2LD.

Figure 29. Time history of $\alpha_{\min }$ for cases 1LD and 2LD. 


\section{VIII.D. Assessment of Computational Cost}

As this research is aimed at making aerothermoelastic analysis of HSVs feasible in a control design and simulation setting, one important metric is the computational cost associated with the reduced-order framework. While reducing the number of aerothermoelastic states for the purpose of control studies is the prime motivation for this work, simulation time is a practical consideration that must be taken into account. The average computational time for one iteration of each of the various components of the solution is given in Table 7 for both the full-order and reduced-order models. Note that the heat flux iteration for the reduced-order model also consists of calculation of the local skin friction coefficients for each element at the outer surface using the Eckert reference temperature method. The data assumes that the size of the aeroelastic time step is equal to the size of the heat transfer time step, and that one complete aerothermoelastic iteration contains ten aeroelastic subiterations $\left(\Delta t_{A E}=\Delta t_{H T}, \Delta t_{A T}=10 \Delta t_{A E}\right)$. Therefore, one heat transfer iteration contains ten time steps of the thermal solution. The computational times for the unsteady aerodynamic iteration and aerodynamic force calculation are the same in both cases because the same model is used in both the full-order and reduced-order solutions. Table 7 also gives the average computational time to complete one full aerothermoelastic iteration for both the full-order and reduced-order models. Based on the relative time step sizes assumed, one aerothermoelastic iteration includes one heat flux and heat transfer iteration and ten structural iterations, unsteady aerodynamic iterations, and aerodynamic force calculations. The last column in Table 7 gives the ratio of the computational time for the full-order solution to that for the reduced-order solution.

The component of the reduced-order aerothermoelastic solution with the greatest computational cost is the structural iteration. Though a fixed basis is used for the structural dynamic response, the current framework still requires that finite element solver be called at every structural iteration to update the $8,074 \times 8,074$ physical stiffness matrix and pre- and post-multiply by the structural reference modes to obtain the generalized stiffness matrix. Recall that the physical stiffness update consists of updating the conventional stiffness matrix based on the temperature-dependence of material properties as well as solving a linear static problem to generate the geometric stiffness matrix. There exists potential for significant improvement in overall computational cost by utilizing reduced-order techniques to update the generalized stiffness matrix directly based on the temperature distribution.

Table 7. Comparison of computational cost between full-order and reduced-order models.

\begin{tabular}{l|c|c|c}
\hline \hline & \multicolumn{2}{|c|}{ CPU Time / Iteration $[\mathrm{s}]$} & \\
\hline Iteration Type & Full-Order & Reduced-Order & Ratio \\
\hline Heat Flux Iteration & $375^{\mathrm{a}}$ & $0.0924^{\mathrm{b}}$ & 4,058 \\
\hline Heat Transfer Iteration (10 time steps) & $0.215^{\mathrm{b}}$ & $0.00246^{\mathrm{b}}$ & 87.4 \\
\hline Structural Iteration (1 time step) & $6.23^{\mathrm{b}}$ & $4.41^{\mathrm{b}}$ & 1.41 \\
\hline Unsteady Aerodynamic Iteration & \multicolumn{2}{|c|}{$0.162^{\mathrm{b}}$} & $\mathrm{N} / \mathrm{A}$ \\
\hline Aerodynamic Force Calculation & & $0.392^{\mathrm{b}}$ & $\mathrm{N} / \mathrm{A}$ \\
\hline Aerothermoelastic Iteration $\left(\Delta t_{A E}=\Delta t_{H T}, \Delta t_{A T}=10 \Delta t_{A E}\right)$ & $443^{\mathrm{c}}$ & $50.0^{\mathrm{b}}$ & 8.86 \\
\hline \hline
\end{tabular}

a 17 2.60-GHz Opteron processors, 2.0 GB RAM.

b 1 2.53-GHz Intel Xeon E5540 processor, 3.0 GB RAM.

${ }^{\mathrm{c}}$ Predicted time.

\section{Concluding Remarks}

A time-marching aerothermoelastic framework is presented which makes use of reduced-order aerothermal, heat transfer, and structural dynamic models for computationally efficient simulation of hypersonic vehicles. The various components of the framework are fully coupled to capture the interactions among the various disciplines in a dynamic sense. The major components of the framework used for the various disciplines consist of:

- Aerothermal: CFD-based kriging surrogate with Latin Hypercube Sampling to calculate the aerody- 
namic heat flux at the outer surface

- Heat Transfer: Reduced-order POD modal formulation to obtain the transient temperature distribution

- Structural Dynamics: Ritz-based modal formulation to calculate the transient structural dynamic response

- Unsteady Aerodynamics: Piston theory unsteady flow solver with shock-expansion analysis to obtain steady flow conditions by turning flow through angle of attack

The time-step scheduling of the aerothermal, heat transfer, and structural dynamics solutions are intentionally partitioned to allow for different size time steps so that the different time scales governing the various processes can be resolved efficiently. The aerothermoelastic framework is applied to a representative HSV control surface as such a component is expected to have a strong impact on the overall vehicle flight dynamics.

The thermal and structural ROMs are validated against the corresponding high-fidelity finite element models for two sets of flight conditions. Though the thermal ROM has some difficulty capturing the temperature response when started from low initial temperatures, the maximum NRMSE for both validation cases is below $6.5 \%$. Comparison of the displacements between the high-fidelity and reduced-order structural models shows that though the initial high-frequency oscillations are not well captured by the structural ROM, the error of the six-mode model decreases dramatically after approximately $10 \mathrm{~s}$. The addition of a seventh mode based on the static displacements in the reference thermal state was found to improve the accuracy of the structural ROM by up to $9 \%$ for one of the validation cases. Comparison of high-fidelity simulations with different size aeroelastic time steps shows that although small aeroelastic time steps are needed to capture high-frequency oscillations, larger aeroelastic time-step sizes may be suitable to obtain the response in cases where thermal loads are dominant over aerodynamic loads. The reduced-order aerothermodynamic model is compared to 500 full-order CFD solutions for the aerodynamic heat flux over the control surface. The average errors were low, on the order of $2 \%$, while the overall maximum error was $27 \%$. The computational efficiency of the model is significant compared to full-order solutions.

Assessment of the transient lift and drag for four different sets of flight conditions provides insight into the impact of aerothermoelastic effects on the total aerodynamic forces. Of the two cruise trajectories considered, the maximum absolute relative changes in total lift and drag are $8 \%$ and $17 \%$, respectively. Of the two ascent trajectories, the maximum absolute relative changes in total lift and drag are $3 \%$ and $21 \%$, respectively. In general it appears that aerothermoelasticity has a larger effect on total drag than total lift. An iterative routine based on the angle of attack necessary to match the lift of the elastic control surface to that of the rigid control surface for the two cruise trajectories is a step toward analysis of HSV controllability under fully coupled aerothermoelastic effects. Of the two cruise trajectories considered here, the maximum deperature from the initial angle of attack is found to be $7.4 \%$. However, this value is likely to be highly dependent on flight conditions and further study can provide insight into necessary variation in angle of attack for various maneuevers and trajectories.

\section{Acknowledgment}

This work was supported by the Michigan-AFRL Collaborative Center in Control Science (MACCCS) under grant number FA 8650-07-2-3744 (Air Force Research Laboratory/Air Vehicles Directorate) with Michael Bolender as program manager. This research is also funded in part by a NASA award NNX08AB32A with Don Soloway and Jorge Bardina as program managers. Finally, this work was supported in part by an allocation of computing time from the Ohio Supercomputer Center.

\section{References}

\footnotetext{
${ }^{1}$ Anderson, Jr., J. D., Hypersonic and High-Temperature Gas Dynamics, McGraw-Hill, 1989.

${ }^{2}$ Bolender, M. A. and Doman, D. B., "Nonlinear Longitudinal Dynamical Model of an Air-Breathing Hypersonic Vehicle," Journal of Spacecraft and Rockets, Vol. 44, No. 2, March-April 2007, pp. 374-387.

${ }^{3}$ Bertin, J. J., Hypersonic Aerothermodynamics, American Institute of Aeronautics and Astronautics, Reston, Virgina, 1994.

${ }^{4}$ McNamara, J. J. and Friedmann, P. P., "Aeroelastic and Aerothermoelastic Analysis of Hypersonic Vehicles: Current Status and Future Trends," Proceedings of the 48th AIAA/ASME/ASCE/AHS/ASC Structures, Structural Dynamics, and Materials Conference, Honolulu, Hawaii, AIAA 2007-2013.
} 
${ }^{5}$ Chavez, F. R. and Schmidt, D. K., "Analytical Aeropropulsive/Aeroelastic Hypersonic-Vehicle Model with Dynamic Analysis," Journal of Guidance, Control, and Dynamics, Vol. 17, No. 6, March-April 2007, pp. 1308-1319.

${ }^{6}$ Bilimoria, K. D. and Schmidt, D. K., "Integrated Development of the Equations of Motion for Elastic Hypersonic Vehicles," Journal of Guidance, Control, and Dynamics, Vol. 18, No. 1, January-February 1995, pp. 73-81.

${ }^{7}$ Parker, J. T., Serrani, A., Yurkovich, S., Bolender, M. A., and Doman, D. B., "Control-Oriented Modeling of an AirBreathing Hypersonic Vehicle," Journal of Guidance, Control, and Dynamics, Vol. 30, No. 3, May-June 2007, pp. 856-869.

${ }^{8}$ Skujins, T., Cesnik, C. E. S., Oppenheimer, M. W., and Doman, D. B., "Applicability of an Analytical Shock/Expansion Solution to the Elevon Control Effectiveness for a 2-D Hypersonic Vehicle Configuration," Proceedings of the 2008 AIAA Atmospheric Flight Mechanics Conference, Honolulu, Hawaii, AIAA 2008-6384.

${ }^{9}$ Oppenheimer, M. W. and Doman, D. B., "A Hypersonic Vehicle Model Developed with Piston Theory," Proceedings of the 2006 AIAA Atmospheric Flight Mechanics Conference, Keystone, Colorado, AIAA 2006-6637.

${ }^{10}$ Oppenheimer, M. W., Skujins, T., Bolender, M. A., and Doman, D. B., "A Flexible Hypersonic Vehicle Model Developed with Piston Theory," Proceedings of the 2007 AIAA Atmospheric Flight Mechanics Conference, Hilton Head, South Carolina, AIAA 2007-6396.

${ }^{11}$ Bolender, M. A., Oppenheimer, M. W., and Doman, D. B., "Effects of Unsteady and Viscous Aerodynamics on the Dynamics of a Flexible Air-Breathing Hypersonic Vehicle," Proceedings of the 2007 AIAA Atmospheric Flight Mechanics Conference, Hilton Head, South Carolina, AIAA 2007-6397.

${ }^{12}$ Culler, A. J., Williams, T., and Bolender, M. A., "Aerothermal Modeling and Dynamic Analysis of a Hypersonic Vehicle," Proceedings of the 2007 AIAA Atmospheric Flight Mechanics Conference, Hilton Head, South Carolina, AIAA $2007-6395$.

${ }^{13}$ Mei, C., Motagaly, K. A., and Chen, R., "Review of Nonlinear Panel Flutter at Supersonic and Hypersonic Speeds," Applied Mechanics Review, Vol. 52, No. 10, October 1999, pp. 321-332.

${ }^{14}$ Pourtakdoust, S. H. and Fazelzadeh, S. A., "Nonlinear Aerothermoelastic Behavior of Skin Panel with Wall Shear Stress Effect," Journal of Thermal Stresses, Vol. 28, No. 2, 2005, pp. 147-169.

${ }^{15}$ Gee, D. J. and Sipcic, S. R., "Coupled Thermal Model for Nonlinear Panel Flutter," AIAA Journal, Vol. 37, No. 5, May 1999, pp. 642-650.

${ }^{16}$ Dechaumphai, P., Thornton, E. A., and Wieting, A. R., "Flow-Thermal-Structural Study of Aerodynamically Heated Leading Edges," Journal of Spacecraft and Rockets, Vol. 26, No. 4, July-August 1989, pp. 201-209.

${ }^{17}$ Thornton, E. A. and Dechaumphai, P., "Coupled Flow, Thermal, and Structural Analysis of Aerodynamically Heated Panels," Journal of Aircraft, Vol. 25, No. 11, November 1988, pp. 1052-1059.

${ }^{18}$ Kontinos, D., "Coupled Thermal Analysis Method with Application to Metallic Thermal Protection Panels," Journal of Thermophysics and Heat Transfer, Vol. 2, No. 11, April-June 1997, pp. 173-181.

${ }^{19}$ Praveen, G. N. and Reddy, J. N., "Nonlinear Transient Thermoelastic Analysis of Functionally Graded Ceramic-Metal Plates," Int. J. Solids Structures, Vol. 35, No. 33, 1998, pp. 4457-4476.

${ }^{20}$ K. -J. Sohn and J. -H. Kim, "Structural Stability of Functionally Graded Panels Subjected to Aero-Thermal Loads," Composite Structures, Vol. 82, No. 3, 2008, pp. 317-325.

${ }^{21}$ Ibrahim, H. H., Tawfik, M., and Al-Ajmi, M., "Thermal Buckling and Nonlinear Flutter Behavior of Functionally Graded Material Panels," Journal of Aircraft, Vol. 44, No. 5, 2007, pp. 1610-1618.

${ }^{22}$ Navazi, H. M. and Haddadpour, H., "Aero-Thermoelastic Stability of Functionally Graded Plates," Composite Structures, Vol. 80 , No. 4, 2007, pp. 580-587.

${ }^{23}$ Haddadpour, H., Navazi, H. M., and Shadmehri, F., "Nonlinear Oscillations of a Fluttering Functionally Graded Plate," Composite Structures, Vol. 79, No. 2, 2007, pp. 242-250.

${ }^{24}$ Prakash, T. and Ganapathi, M., "Supersonic Flutter Characteristics of Functionally Graded Flat Panels Including Thermal Effects," Composite Structures, Vol. 72, No. 1, 2006, pp. 10-18.

${ }^{25}$ McNamara, J. J., Friedmann, P. P., Powell, K. G., and Thuruthimattam, B. J., "Aeroelastic and Aerothermoelastic Behavior in Hypersonic Flow," AIAA Journal, Vol. 46, No. 10, October 2008, pp. 2591-2610.

${ }^{26}$ Culler, A. J. and McNamara, J. J., "Studies on Fluid-Thermal-Structural Coupling for Aerothermoelasticity in Hypersonic Flow," AIAA Journal, Accepted 2010. Preprint available upon request.

${ }^{27}$ Culler, A. J. and McNamara, J. J., "Coupled Flow-Thermal-Structural Analysis for Response Prediction of Hypersonic Vehicle Skin Panels," Proceedings of the 51st AIAA/ASME/ASCE/AHS/ASC Structures, Structural Dynamics, and Material Conference, Orlando, Florida, AIAA 2010-2965.

${ }^{28}$ Falkiewicz, N. J. and Cesnik, C. E. S., "Proper Orthogonal Decomposition for Reduced-Order Thermal Solution in Hypersonic Aerothermoelastic Simulations," Proceedings of the 51st AIAA/ASME/ASCE/AHS/ASC Structures, Structural Dynamics, and Material Conference, Orlando, Florida, AIAA 2010-2798.

${ }^{29}$ Falkiewicz, N. J., Cesnik, C. E. S., Bolender, M. A., and Doman, D. B., "Thermoelastic Formulation of a Hypersonic Vehicle Control Surface for Control-Oriented Simulation," Proceedings of the 2009 AIAA Guidance, Navigation, and Control Conference, Chicago, Illinois, AIAA 2009-6284.

${ }^{30}$ Falkiewicz, N. J. and Cesnik, C. E. S., "A Reduced-Order Modeling Framework for Integrated Thermo-Elastic Analysis of Hypersonic Vehicles," Proceedings of the 50th AIAA/ASME/ASCE/AHS/ASC Structures, Structural Dynamics, and Materials Conference, Palm Springs, California, AIAA 2009-2308.

${ }^{31}$ Culler, A. J., Crowell, A. R., and McNamara, J. J., "Studies on Fluid-Structural Coupling for Aerothermoelasticity in Hypersonic Flow," Proceedings of the 50th AIAA/ASME/ASCE/AHS/ASC Structures, Structural Dynamics, and Materials Conference, Palm Springs, California, AIAA 2009-2364.

${ }^{32}$ Crowell, A. R., Culler, A. J., and McNamara, J. J., "Two-Way Coupled Aerothermoelastic Modeling and Simulation of Hypersonic Vehicle Structures," Proceedings of the International Forum on Aeroelasticity and Structural Dynamics, Seattle, WA, June 2009, IFASD-2009-94.

${ }^{33}$ McNamara, J. J., Culler, A. J., and Crowell, A. R., "Aerothermoelastic Modeling Considerations for Hypersonic Vehicles," AIAA Paper 2009-7397, October 2009. 
${ }^{34}$ Crowell, A. R., McNamara, J. J., Kecskemety, K. M., and Goerig, T. W., "A Reduced Order Aerothermodynamic Modeling Framework for Hypersonic Aerothermoelasticity," Proceedings of the 51st AIAA/ASME/ASCE/AHS/ASC Structures, Structural Dynamics, and Material Conference, Orlando, Florida, AIAA 2010-2798.

${ }^{35}$ Huebner, K., Dewhirst, D., Smith, D., and Byrom, T., The Finite Element Method for Engineers, John Wiley \& Sons, Inc., 4th ed., 2001.

${ }^{36}$ Chandrupatla, T. and Belegundu, A., Introduction to Finite Elements in Engineering, Prentice-Hall, Inc., 3rd ed., 2002.

${ }^{37}$ Dhondt, G. D. C., The Finite Element Method for Three-Dimensional Thermomechanical Applications, John Wiley \& Sons, 2004 .

${ }^{38}$ Çengel, Y. A. and Turner, R. H., Fundamentals of Thermal-Fluid Sciences, McGraw-Hill, Inc., 2001.

${ }^{39}$ Holman, J. P., Heat Transfer, McGraw-Hill, Inc., 8th ed., 1997.

${ }^{40}$ Holmes, P., Lumley, J., and Berkooz, G., Turbulence, Coherent Structures, Dynamical Systems and Symmetry, Cambridge University Press, 1996.

${ }^{41}$ Liang, Y. C., Lee, H. P., Lim, S. P., Lin, W. Z., Lee, K. H., and Wu, C. G., "Proper Orthogonal Decomposition and its Applications-Part I: Theory," Journal of Sound and Vibration, Vol. 252, No. 3, 2002, pp. 527-544.

${ }^{42}$ Lumley, J. L., "The Structure of Inhomogeneous Turbulent Flows," Atmospheric Turbulence and Radio Wave Propagation, edited by A. Yaglon and V. Tatarsky, Nauka, Moscow, 1967, pp. 166-178.

${ }^{43}$ Krysl, P., Lall, S., and Marsden, J. E., "Dimensional Model Reduction in Non-Linear Finite Element Dynamics of Solids and Structures," International Journal for Numerical Methods in Engineering, Vol. 51, 2001, pp. 479-504.

${ }^{44}$ Placzek, A., Tran, D., and Ohayon, R., "Hybrid proper orthogonal decomposition formulation for linear structural dynamics," Journal of Sound and Vibration, Vol. 318, No. 4-5, December 2008, pp. 943-964.

${ }^{45}$ Ahlman, D., Söderlund, F., Jackson, J., Kurdila, A., and Shyy, W., "Proper Orthogonal Decomposition for TimeDependent Lid-Driven Cavity Flows," Numerical Heat Transfer B., Vol. 42, No. 4, 2002, pp. 285-306.

${ }^{46}$ Atwell, J. A. and King, B. B., "Proper Orthogonal Decomposition for Reduced Basis Feedback Controllers for Parabolic Equations," Mathematical and Computer Modelling, Vol. 33, 2001, pp. 1-19.

${ }^{47}$ Sirovich, L., "Turbulence and Dynamics of Coherent Structures, Part I: Coherent Structures," Quarterly of Applied Mathematics, Vol. XLV, 1987, pp. 561-571.

${ }^{48}$ Bialecki, R. A., Kassab, A. J., and Fic, A., "Proper Orthogonal Decomposition and Modal Analysis for Acceleration of Transient FEM Thermal Analysis," International Journal for Numerical Methods in Engineering, Vol. 62, No. 6, 14 Feb. 2005, pp. $774-797$.

${ }^{49}$ Gunzburger, M. and Peterson, J., "Reduced-Order Modeling of Complex Systems with Multiple System Parameters," Large-Scale Scientific Computing, 5th International Conference, Sozopol, Bulgaria, edited by I. Lirkov, S. Margenov, and J. Wasniewski, Vol. LNCS 3743, Springer Verlag, 2006, pp. 15-27.

${ }^{50}$ Trefethen, L. N. and Bau, III, D., Numerical Linear Algebra, Society for Industrial and Applied Mathematics, 1997.

${ }^{51}$ Heath, M. T., Scientific Computing : An Introductory Survey, McGraw-Hill, 2nd ed., 2002.

${ }^{52}$ Chatterjee, A., "An introduction to the proper orthogonal decomposition," Current Science, Vol. 78, No. 7, April 2000, pp. 808-817.

${ }^{53}$ Wood, W. L. and Lewis, R. W., "A Comparison of Time Marching Schemes for the Transient Heat Conduction Equation," Int. J. Numer. Methods Eng., Vol. 9, No. 3, 1975, pp. 679-689.

${ }^{54}$ Hirsch, C., Numerical Computation of Internal \& External Flows, Butterworth-Heinemann, 2nd ed., 2007.

${ }^{55}$ Hughes, T. J. R., "Unconditionally Stable Algorithms for Nonlinear Heat Conduction," Computer Methods in Applied Mechanics and Engineering, Vol. 10, 1977, pp. 135-139.

${ }^{56}$ MSC.Software Corporation, Santa Ana, CA, MSC.Nastran Basic Dynamic Analysis User's Guide: Version 68, 2004.

${ }^{57}$ Craig, R. R. and Kurdila, A. J., Fundamentals of Structural Dynamics, John Wiley \& Sons, Inc., 2nd ed., 2006.

${ }^{58}$ Lophaven, S. N., Nielsen, H. B., and Sondergaard, J., "DACE A Matlab Kriging Toolbox Version 2.0," Technical Report IMM-TR-2002-12, August 2002.

${ }^{59}$ Sacks, J., Welch, W. J., Mitchell, T. J., and Wynn, H. P., "Design and Analysis of Computer Experiments," Statistical Science, Vol. 4, No. 4, 1989, pp. 409-435.

${ }^{60}$ Chung, H. S. and Alonso, J. J., "Comparison of Approximation Models with Merit Functions for Design Optimization," AIAA Paper 2000-4754, September 2000.

${ }^{61}$ Kolonay, R. M. and Lambe, L. A., "Determination of the Optimal Set of Parameters of a Kriging Model for an Euler Based Induced Drag Function," Proceedings of the International Forum on Aeroelasticity and Structural Dynamics, No. IFASD2009-005, June 2009.

${ }^{62}$ Simpson, T. W., Mauery, T. M., Korte, J. J., and Mistree, F., "Kriging Models for Global Approximation in SimulationBased Multidisciplinary Design Optimization," AIAA Journal, Vol. 39, No. 12, December 2001, pp. 2233-2241.

${ }^{63} \mathrm{Glaz}$, B., Active/Passive Optimization of Helicopter Rotor Blades for Improved Vibration, Noise, and Performance Characteristics, Ph.D. Thesis, University of Michigan, Ann Arbor, Michigan, 2008.

${ }^{64}$ Kumano, T., Jeong, S., Obayashi, S., Ito, Y., Hatanaka, K., and Morino, H., "Multidisciplinary Design Optimization of Wing Shape for a Small Jet Aircraft Using Kriging Model," AIAA Paper 2006-932, January 2006.

${ }^{65}$ Lam, X. B., Kim, Y. S., Hoang, A. D., and Park, C. W., "Coupled Aerostructural Design Optimization Using the Kriging Model and Integrated Multiobjective Optimization Algorithm," Journal of Optimization Theory and Applications, DOI 10.1007/s10957-009-9520-9, March 2009.

${ }^{66}$ Lighthill, M., "Oscillating Airfoils at High Mach Number," Journal of the Aeronautical Sciences, Vol. 20, No. 6, June 1953, pp. 402-406.

${ }^{67}$ Ashley, H. and Zartarian, G., "Piston Theory - A New Aerodynamic Tool for the Aeroelastician," Journal of the Aeronautical Sciences, Vol. 23, No. 12, 1956, pp. 1109-1118.

${ }^{68}$ Anderson, J. D., Modern Compressible Flow: With Historical Perspective, McGraw-Hill, Boston, 3rd ed., 2003. 
${ }^{69}$ Thuruthimattam, B. J., Friedmann, P. P., McNamara, J. J., and Powell, K. G., "Modeling Approaches to Hypersonic Aeroelasticity," 2002 ASME International Mechanical Engineering Congress and Exposition, New Orleans, Louisiana, November IMECE/2002-32943.

${ }^{70}$ Pendleton, E., Moster, G., and Farmer, M., "Transonic Aeroelastic Models of Hypersonic Highly Swept Lifting Surfaces: Design, Analyses, and Test," Proceedings of the 35th AIAA/ASME/ASCE/AHS/ASC Structures, Structural Dynamics, and Materials Conference, Hilton Head, South Carolina, AIAA 1994-1489-574.

${ }^{71}$ Pendleton, E., Moster, G., and Keller, D., "Transonic Aeroelastic Models of Highly Swept Hypersonic Lifting Surfaces," Journal of Aircraft, Vol. 32, No. 6, Nov. - Dec. 1995, pp. 1169-1176.

${ }^{72}$ Spain, C. V., Zeiler, T. A., Bullok, E. P., and Hodge, J. S., "A Flutter Investigation of All-Moveable NASP-Like Wings at Hypersonic Speeds," Proceedings of the 34th AIAA/ASME/ASCE/AHS/ASC Structures, Structural Dynamics, and Materials Conference, La Jolla, California, AIAA-93-1315.

${ }^{73}$ McNamara, J. J., Aeroelastic and Aerothermoelastic Behavior of Two and Three Dimensional Lifting Surfaces in Hypersonic Flow, Ph.D. thesis, University of Michigan, Ann Arbor, Michigan, 2005.

${ }^{74}$ Shih, P., Prunty, J., and Mueller, R., "Thermostructural Concepts for Hypervelocity Vehicles," Journal of Aircraft, Vol. 28, No. 5, May 1991, pp. 337-345.

${ }^{75}$ Myers, D., Martin, C., and Blosser, M., "Parametric Weight Comparison of Advanced Metallic, Ceramic Tile, and Ceramic Blanket Thermal Protection Systems," Tech. Rep. NASA TM-210289, Langley Research Center, June 2000.

${ }^{76}$ Orton, G. and Scuderi, L., "A Hypersonic Cruiser Concept for the 21st Century," Proceedings of the 1998 World Aviation Conference, Anaheim, California, AIAA 1998-5525.

${ }^{77}$ Wieting, A., Dechaumphai, P., Bey, K., Thornton, E., and Morgan, K., "Application of Integrated Fluid-ThermalStructural Analysis Methods," Thin-Walled Structures, Vol. 11, No. 1-2, 1991, pp. 1-23.

${ }^{78}$ Leatherwood, J., Clevenson, S., Powell, A., and Daniels, E., "Acoustic Testing of High-Temperature Panels," Journal of Aircraft, Vol. 29, No. 6, Nov. - Dec. 1992, pp. 1130-1136.

${ }^{79}$ Blevins, R., Bofilios, D., Holehouse, I., Hwa, V., Tratt, M., Laganelli, A., Pozefsky, P., and Pierucii, M., "Thermo-VibroAcoustic Loads and Fatigue of Hypersonic Flight Vehicle Structure," Tech. Rep. AFRL-RB-WP-TR-2009-3139, Air Force Research Laboratory, June 2009

${ }^{80}$ Ellis, D., Pagel, L., and Schaeffer, D., "Design and Fabrication of a Radiative Actively Cooled Honeycomb Sandwich Structural Panel for a Hypersonic Aircraft," Tech. Rep. NASA-CR-2957, NASA, March 1978

${ }^{81}$ Ko, W. and Fields, R., "Thermal Stress Analysis of Space Shuttle Orbiter Subjected to Reentry Aerodynamic Heating,"

Tech. Rep. NASA-TM-88286, NASA, Oct. 1987.

${ }^{82}$ Leyens, C. and Peters, M., Titanium and Titanium Alloys, Wiley-VHC, 2003.

${ }^{83}$ Noor, A. K., editor, Structures Technology: Historical Perspective and Evolution, American Institute of Aeronautics and Astronautics, 1998

${ }^{84}$ Department of Defense, Military Handbook - MIL-HDBK-5H: Metallic Materials and Elements for Aerospace Vehicle Structures, December 1998.

${ }^{85}$ Welsch, G., Boyer, R., and Collings, E. W., Materials Properties Handbook: Titanium Alloys, ASM International, 1994. 86 "Titanium IMI 834, " http://http://www.matweb.com/search/DataSheet.aspx?MatGUID= a74096c99aa6486382a9c9e1be0883c4\&ckck=1, Last Accessed 9/3/2009.

${ }^{87}$ Krist, S. L., Biedron, R. T., and Rumsey, C. L., "CFL3D User's Manual (Version 5.0)," NASA TM 1998-208444, 1997, CFL3D Version 6.0 update: http://cfl3d.larc.nasa.gov/Cfl3dv6/cfl3dv6.html.

${ }^{88}$ Bartels, R. E., Rumsey, C. L., and Biedron, R. T., "CFL3D User's Manual - General Usage and Aeroelastic Analysis (Version 6.4)," NASA TM 2006-214301, 2006.

${ }^{89}$ Menter, F. R. and Rumsey, C. L., "Assessment of Two-Equation Turbulence Models for Transonic Flows," AIAA Paper 1994-2343, June 1994.

${ }^{90}$ Roy, C. J. and Blottner, F. G., "Methodology for Turbulence Model Validation: Application to Hypersonic Flows," Journal of Spacecraft and Rockets, Vol. 40, No. 3, May-June 2003, pp. 313-325.

${ }^{91}$ Rumsey, C. L., "Compressibility Considerations for k-w Turbulence Models in Hypersonic Boundary Layer Applications," NASA/TM2009-215705, April 2009.

${ }^{92}$ Eckert, E. R. G., "Engineering Relations for Friction and Heat Transfer to Surfaces in High Velocity Flow," Journal of the Aeronautical Sciences, Vol. 22, No. 8, March 23, 1955, pp. 585-587.

${ }^{93}$ Eckert, E. R. G., "Engineering Relations for Heat Transfer and Friction in High-Velocity Laminar and Turbulent Boundary-Layer Flow Over Surfaces with Constant Pressure and Temperature," Transactions of the American Society of Mechanical Engineers, Vol. 78, No. 6, August 1956 